\title{
Correspondência do tipo Galois para ações de álgebras de Hopf em álgebras primas
}

\author{
Octávio Bernardes Ferreira Neto \\ DisSERTAÇÃO APRESENTADA \\ $\mathrm{AO}$ \\ Instituto de Matemática e Estatística \\ DA \\ Universidade DE SÃo Paulo \\ PARA \\ OBTENÇÃO DO TÍTULO \\ $\mathrm{DE}$ \\ Mestre em CiênCIAS \\ Área de Concentração: Matemática
Orientador: Profa. Dra. Lucia Satie Ikemoto Murakami
}

São Paulo, agosto de 2008. 



\title{
Correspondência do tipo Galois para ações de álgebras de Hopf em álgebras primas
}

\author{
Este exemplar corresponde à redação \\ final da dissertação devidamente corrigida \\ e defendida por Octávio Bernardes Ferreira Neto \\ e aprovada pela Comissão Julgadora.
}

Banca Examinadora:

- Profa. Dra. Lucia Satie Ikemoto Murakami (Presidente) - IME-USP.

- Prof. Dr. Vitor de Oliveira Ferreira - IME-USP.

- Prof. Dr. Antonio Paques - UFRGS. 

Dedico este trabalho a duas pessoas.

A primeira por ter me motivado a começar.

A segunda por ter me dado forças para terminar.

Daniel e Matheus. 



\section{Agradecimentos}

Inicialmente, agradeço aos meus pais, Márcia e Márcio, que sempre cultivaram e demonstraram de forma incisiva a importância da educação na vida de uma pessoa. Passei a acreditar que a educação pela educação já basta como justificativa.

Gostaria de agradecer ao Instituto de Matemática e Estatística por duas razões, a primeira por ter me aceitado como aluno mesmo não tendo graduado em matemática, e a segunda por ter um ambiente tranquilo e receptivo, o qual tive a oportunidade de desfrutar e que me era sempre uma fonte de inspiração.

Agradeço também aos diversos colegas e professores que me ajudaram de alguma forma nessa jornada, seja através de conversas inspiradoras, ou seja através da transferência de conhecimento e direcionamento. Não citarei nomes para não correr o risco de cometer alguma injustiça.

Finalmente, agradeço à minha orientadora, Profa. Dra. Lucia Satie Ikemoto Murakami, por ter acreditado em mim desde o começo e por ter continuado a acreditar mesmo quando parecia que não seria possível a conclusão deste trabalho.

Obrigado a vocês! 


\section{Resumo}

Neste trabalho demonstramos um teorema da correspondência do tipo Galois para ações de álgebras de Hopf pontuais de dimensão finita em álgebras primas. A correspondência acontece entre subálgebras racionalmente completas e comódulo subálgebras. As subálgebras racionalmente completas são subálgebras da álgebra prima, enquanto os comódulo subálgebras são comódulo subálgebras do produto smash entre o centralizador da álgebra prima em sua álgebra de quocientes de Martindale simétrica e a álgebra de Hopf.

Palavras-chave: ações de álgebras de Hopf; correspondência Hopf-Galois; estrutura de coálgebras 


\section{Abstract}

A Galois-type correspondence theorem for prime algebras acted upon by a finite dimensional pointed Hopf algebra is proved. The correspondence involves rationally complete subalgebras and comodule subalgebras. The rationally complete subalgebras are subalgebras of the prime algebra, while the comodule subalgebras are comodule subalgebras of the smash product between the centralizer of the prime algebra in its symmetric Martindale quotient algebra and the Hopf algebra.

Keywords: Hopf algebra actions; Hopf-Galois correspondence; coalgebra structures. 


\section{Sumário}

Lista de Símbolos $\quad$ ix

Introdução

1 Preliminares $\quad 1$

1.1 Introdução . . . . . . . . . . . . . . . . . . . . . . . . . . 1

1.2 Definições básicas . . . . . . . . . . . . . . . . . . . . . 1

1.3 Álgebras de Hopf . . . . . . . . . . . . . . . . . . . . . 5

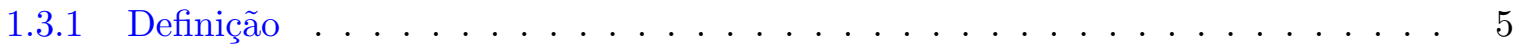

1.3.2 Ações, produto cruzado e o produto smash . . . . . . . . . . . . . . 14

1.3.3 Comódulos e comódulo álgebras . . . . . . . . . . . . . . . . . 18

1.3.4 Coálgebras pontuais e a filtração co-radical . . . . . . . . . . . . . . . 22

1.3 .5 Integrais . . . . . . . . . . . . . . . . . 24

1.4 A álgebra de quocientes de Martindale . . . . . . . . . . . . . . . . . . . . 27

1.5 Ações de álgebra de Hopf em álgebras primas . . . . . . . . . . . . . . . . . . . 29

2 Mais sobre coálgebras $\quad 31$

2.1 Introdução . . . . . . . . . . . . . . . . . . . . . . 31 
2.2 Alguns coideais especiais . . . . . . . . . . . . . . . . . . 31

2.2 .1 Os coideais da forma $L(c)$ e $R(c) \ldots \ldots \ldots \ldots \ldots \ldots \ldots \ldots$

2.2 .2 Os coideais da forma $H^{Y} \ldots \ldots \ldots \ldots \ldots$

2.3 A estrutura de coálgebras pontuais . . . . . . . . . . . . . . . . 38

$\begin{array}{lll}3 & \text { As ações X-externas } & 47\end{array}$

3.1 Introdução . . . . . . . . . . . . . . . . . . . . . . . . . . . 47

3.2 Propriedades dos produtos smash . . . . . . . . . . . . . . . . . 50

3.3 Definição das ações X-externas . . . . . . . . . . . . . . . . . 56

3.4 Propriedades fundamentais das ações X-externas . . . . . . . . . . . . . . . . 62

4 A injetividade da correspondência de Galois $\quad 73$

4.1 Introdução . . . . . . . . . . . . . . . . . . . . . . . 73

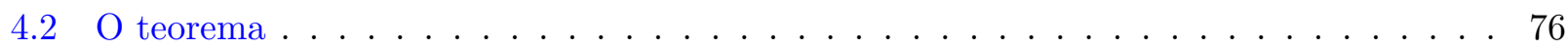

$\begin{array}{lll}5 & \text { A sobrejetividade da correspondência de Galois } & 83\end{array}$

5.1 Introdução . . . . . . . . . . . . . . . . . . . . . . 83

5.2 Propriedades de $H^{K} \ldots \ldots \ldots \ldots \ldots \ldots \ldots \ldots \ldots \ldots$

5.3 Propriedades dos comódulo subálgebras . . . . . . . . . . . . . . . . . 92

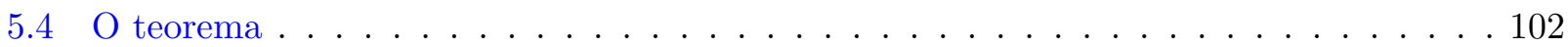

5.5 Considerações finais $\ldots \ldots \ldots \ldots$. . . . . . . . . . . . . . . . 104

$\begin{array}{ll}\text { Referências Bibliográficas } & 107\end{array}$

$\begin{array}{lr}\text { Índice Remissivo } & 109\end{array}$ 


\title{
Lista de Símbolos
}

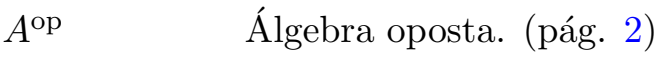 \\ $a \bullet b \quad$ Multiplicação de $a$ e $b$ na álgebra oposta. (pág. 2) \\ $\mathcal{F}(R) \quad$ Conjunto de todos ideais não nulos de $R$. (pág. 3) \\ $X^{Y} \quad$ Centralizador de $Y$ em $X$. (pág. 4) \\ $\operatorname{span}_{k}\left\{m_{i}\right\} \quad$ Subespaço gerado pelos elementos $m_{i}$. (pág. 4) \\ $\tau \quad$ Aplicação twist. (pág. 4) \\ $\operatorname{hom}_{k}(V, W)$ Conjunto das transformações lineares de $V$ em $W$. (pág. 4) \\ $V^{*} \quad$ Espaço dual de $V$. (pág. 4) \\ $\phi^{*} \quad$ Aplicação transposta de $\phi$. (pág. 4) \\ $J^{+} \quad$ Conjunto $J \cap \operatorname{ker}(\varepsilon)$. (pág. 7)

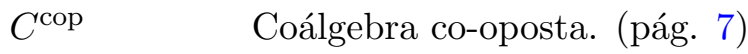

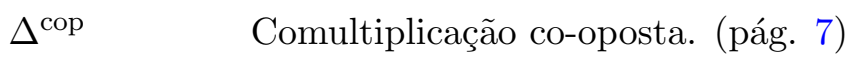 \\ $G(C) \quad$ Conjunto dos elementos group-like de $C$. (pág. 8) \\ $P_{g, h}(C) \quad$ Conjunto dos elementos $g, h$-primitivos de $G(C)$. (pág. 8) \\ $P_{g, h}^{\prime}(C) \quad$ Subespaço de $P_{g, h}(C)$ complementar a $k(g-h)$. (pág. 8) \\ $P(B) \quad$ Conjunto dos elementos primitivos de $B$. (pág. 10) \\ $f * g \quad$ Produto de convolução entre $f$ e $g$. (pág. 11) \\ $S \quad$ Antípoda de $H$. (pág. 12) \\ $\bar{S} \quad$ Inversa de $S$ na composição. (pág. 12) \\ $A^{H} \quad$ Subálgebra de invariantes de $A$ sob a ação de $H$. (pág. 14)
}


$\operatorname{ad}_{1}(h, b)$ Ação adjunta à esquerda de $h$ em $b$. (pág. 14)

$\operatorname{ad}_{\mathrm{r}}(h, b) \quad$ Ação adjunta à direita de $h$ em $b$. (pág. 14)

$A \# H \quad$ Produto smash de $A$ e $H$. (pág. 16)

$A^{\mathrm{co} H} \quad$ Subálgebra de coinvariantes de $A$. (pág. 21)

$L(c) \quad$ Coideal à direita gerado por $c$. (pág. 32)

$R(c) \quad$ Coideal à esquerda gerado por c. (pág. 32) 


\section{Introdução}

O objetivo deste trabalho é demonstrar um teorema de correspondência do tipo Galois para ações de álgebras de Hopf pontuais de dimensão finita em álgebras primas. Esse teorema generaliza vários resultados obtidos no passado, conforme veremos a seguir.

Seja $L: K$ uma extensão de corpos com grupo de Galois $G$, que consiste de todos os automorfismos de $L$ que fixam os elementos de $K$. Considere também $\mathcal{F}$ o conjunto dos corpos intermediários entre $K$ e $L$, e $\mathcal{G}$ o conjunto dos subgrupos de $G$. A teoria clássica da correspondência de Galois diz que há uma correspondência biunívoca entre $\mathcal{F}$ e $\mathcal{G}$.

A teoria clássica da correspondência de Galois pode ser generalizada em duas direções. Por um lado, os corpos podem ser substituídos por anéis comutativos ou não comutativos. Por outro lado, ações de grupo podem ser substituídas por ações de álgebras de Hopf. Observe que, na teoria clássica da correspondência de Galois, $G$ age em $L$ pela aplicação identidade de $G$ em $\operatorname{Aut}(L)$.

Em [Kha91], Kharchenko obteve um teorema da correspondência do tipo Galois para ações de grupos finitos de automorfismos e para ações de álgebras de Lie restritas de dimensão finita, em álgebras primas e semi-primas. Podemos ver o resultado de Kharchenko como ações de uma álgebra de grupo e ações da envolvente universal, ambas álgebras de Hopf cocomutativas e pontuais de dimensão finita, em álgebras primas e semi-primas. Com isso, surge a pergunta se a correspondência não poderia ser generalizada para ações de álgebras de Hopf pontuais de dimensão finita, não necessariamente cocomutativas.

Um dos resultados mais importantes no caminho para se chegar em uma demonstração do teorema da correspondência foi a definição de ação X-externa feita em [Mil95a]. Essa definição foi importante pois generalizou a definição de ação X-externa feita anteriormente por Karchenko. 
Em [Yan97], Yanai descreveu um teorema da correspondência do tipo Galois para o caso em que a álgebra de Hopf é a álgebra de Hopf de Sweedler, que é não cocomutativa e que tem dimensão quatro. Além disso, nesse mesmo artigo, Yanai descreveu quais passos deveriam ser seguidos para se alcançar uma versão da correspondência de Galois para o caso geral envolvendo álgebras de Hopf pontuais de dimensão finita, além de demonstrar alguns resultados básicos.

Demonstrar essa versão do teorema da correspondência de Galois para o caso geral envolvendo ações de álgebras de Hopf pontuais de dimensão finita em álgebras primas é o objetivo deste trabalho. Segue uma breve descrição deste teorema.

Seja $R$ uma álgebra prima. Denotamos por $Q$ a álgebra de quocientes de Martindale simétrica de $R$ e por $K$ o centróide estendido de $R$. Seja $H$ uma álgebra de Hopf pontual de dimensão finita que age em $R$. Por [Coh86], podemos estender essa ação a uma ação de $H$ em $Q$.

De [Mil95b], temos que $K$ é estável sob a ação de $H$ desde que a ação de $H$ em $R$ seja X-externa. Assim, podemos considerar os produtos smash $K \# H \subseteq Q \# H$.

Para quaisquer subconjuntos $V$ e $W$ de uma álgebra qualquer, vamos denotar por $V^{W}$ o centralizador de $W$ em $V$. Segue o enunciado do teorema cuja demonstração é o objetivo desta dissertação.

Teorema. Seja $k$ um corpo de característica zero e seja $H$ uma álgebra de Hopf pontual de dimensão finita sobre $k$ agindo em uma $k$-álgebra prima $R$ de modo que a ação seja $X$-externa. Denotemos por $Q$ a álgebra de quocientes de Martindale simétrica de $R$ e por $K$ o centróide estendido de $R$. Seja $\mathcal{S}$ o conjunto de todos os $H$-comódulo subálgebras $\Lambda$ de $K \# H$ contendo $K$, e seja $\mathcal{F}$ o conjunto de todas as subálgebras racionalmente completas $U$ de $R$ contendo $R^{H}$. Então a aplicação

$$
\begin{aligned}
& \Phi: \mathcal{F} \longrightarrow \mathcal{S} \\
& U \longmapsto(K \# H)^{U}
\end{aligned}
$$

é bijetora com inversa dada por

$$
\begin{aligned}
\Psi: \mathcal{S} & \longrightarrow \mathcal{F} \\
\Lambda & \longmapsto R^{\Lambda}
\end{aligned}
$$

Antes de prosseguir, convém ressaltar que todos os teoremas, proposições e lemas apresentados nesta dissertação tiveram suas origens citadas. Muitos deles tiveram suas hipóteses ligeiramente alteradas e suas demonstrações bastante modificadas com o propósito de traçar um caminho direto, através da compilação e detalhamento de resultados de diversos artigos, até a demonstração do 
teorema da correspondência. Segue uma breve descrição de como este trabalho foi dividido.

No Capítulo 1 apresentamos diversas definições e resultados preliminares para o restante deste trabalho. A maior parte dos resultados não foram demonstrados pois poderíamos perder o foco do assunto principal. Apesar disso, esse capítulo faz com que esta dissertação seja auto-contida. Nos capítulos subsequentes todos os resultados foram demonstrados, com exceção de alguns que foram considerados imediatos.

O Capítulo 2 faz um detalhamento maior dos coideais de uma coálgebra e da estrutura das coálgebras pontuais. Os resultados da seção que trata dos coideais serão utilizados principalmente na demonstração da sobrejetividade da correspondência, enquanto os resultados da seção que lida com a estrutura das coálgebras pontuais serão utilizados por todo o trabalho.

O Capítulo 3 é um capítulo chave, pois traz os resultados e definições fundamentais das ações X-externas. Nele, definimos as ações X-externas e mostramos diversas propriedades deste tipo de ação. Além disso, ao final deste capítulo, já é possível compreender o enunciado do teorema da correspondência.

No Capítulo 4 demonstramos a injetividade da correspondência. Finalmente, no Capítulo 5 demonstramos a sobrejetividade da correspondência e ilustramos a correspondência com um exemplo envolvendo a álgebra de Hopf de Sweedler, cumprindo o objetivo deste trabalho. 


\section{Capítulo 1}

\section{Preliminares}

\subsection{Introdução}

Este capítulo contém diversas definições e resultados que serão fundamentais para os capítulos subsequentes. A menos que indicado, sempre vamos utilizar um corpo fixo $k$ como corpo base para as diversas estruturas presentes neste trabalho, tais como álgebras, coálgebras, produtos tensoriais, entre outros.

Dividimos este capítulo da seguinte forma. A Seção 1.2 traz uma série de definições e resultados básicos sobre álgebras, álgebras primas e algumas notações que serão utilizadas no decorrer do texto. Na Seção 1.3, definimos álgebra de Hopf e enunciamos uma série de propriedades e resultados sobre essa estrutura. Na Seção 1.4 introduzimos as álgebras de quocientes de Martindale e na Seção 1.5 enunciamos uma série de resultados fundamentais sobre ações de álgebra de Hopf em álgebras primas.

\subsection{Definições básicas}

Assumimos que o leitor tenha familiaridade com resultados e propriedades de espaços vetoriais, produtos tensoriais de espaços vetoriais, anéis, módulos, e seus diversos morfismos. Entretanto, gostaríamos de fazer algumas observações sobre produtos tensoriais de espaços vetoriais que, pela sua importância e ampla utilização no decorrer deste trabalho, merecem ser citadas. 
1. Sejam $U$ e $V$ espaços vetoriais sobre um corpo $k$ com bases $\left\{u_{\alpha}\right\}_{\alpha \in A}$ e $\left\{v_{\beta}\right\}_{\beta \in B}$, respectivamente. Então $C=\left\{u_{\alpha} \otimes_{k} v_{\beta}: \alpha \in A, \beta \in B\right\}$ é uma base do $k$-espaço vetorial $U \otimes_{k} V$. Em particular, se $\operatorname{dim}(U)=m$ e $\operatorname{dim}(V)=n$, então $\operatorname{dim}\left(U \otimes_{k} V\right)=m n$.

2. Sejam $X$ e $Y$ espaços vetoriais. Se $A$ e $B$ são subespaços de $X$ e $C$ e $D$ são subespaços de $Y$, então

$$
(A \otimes C) \cap(B \otimes D)=(A \cap B) \otimes(C \cap D) .
$$

3. Sejam $A \subseteq X$ e $B \subseteq Y$ espaços vetoriais. Se $\sum a_{i} \otimes b_{i} \in A \otimes B$, onde $\left\{b_{i}\right\}$ é linearmente independente, então $a_{i} \in A$ e $b_{i} \in B$, para todo $i$. De forma análoga, se $\sum a_{i} \otimes b_{i} \in A \otimes B$, onde $\left\{a_{i}\right\}$ é linearmente independente, então $a_{i} \in A$ e $b_{i} \in B$, para todo $i$.

Vamos agora às definições e resultados sobre álgebras. Dizemos que um espaço vetorial $A$ é uma álgebra se existir uma aplicação bilinear

$$
\begin{aligned}
m: A \times A & \longrightarrow A \\
(a, b) & \longmapsto a b
\end{aligned}
$$

que seja

(i) associativa, isto é, $(a b) c=a(b c)$, para todos $a, b, c \in A$.

(ii) unital, isto é, que exista $1_{A} \in A$ tal que $a 1_{A}=1_{A} a=a$, para todo $a \in A$.

Esta aplicação bilinear é chamada multiplicação de $A$.

Note que para qualquer álgebra $A$ podemos definir a sua álgebra oposta $A^{\mathrm{op}}$, que coincide com $A$ como espaço vetorial, e cuja multiplicação é dada por $a \bullet b=b a$, para todos $a, b \in A^{\mathrm{op}}$.

Um morfismo de uma álgebra $A$ em uma álgebra $B$ é uma transformação linear $\varphi: A \longrightarrow B$ tal que $\varphi(a b)=\varphi(a) \varphi(b)$, para todos $a, b \in A$, e ainda $\varphi\left(1_{A}\right)=1_{B}$.

Seja $A$ uma álgebra com estrutura de espaço vetorial $(A,+)$. Um subconjunto $I \subseteq A$ é chamado ideal se

(i) $(I,+)$ for um subespaço de $(A,+)$.

(ii) $r x \in I$ e $x r \in I$, para todo $x \in I$ e todo $r \in R$. 
Observação. Em (ii) se tivermos somente que $r x \in I$, para todo $x \in I$ e todo $r \in R$, dizemos que $I$ é um ideal à esquerda. E, se tivermos somente que $x r \in I$, para todo $x \in I$ e todo $r \in R$, dizemos que $I$ é um ideal à direita.

Dizemos que uma álgebra $R$ é prima se, para quaisquer ideais $I, J$ não nulos de $R$ tivermos que $I J$ é um ideal não nulo de $R$. Observe que uma álgebra $R$ é prima se, e somente se, para quaisquer elementos $x, y \in R$ tais que $x R y=0$, tivermos $x=0$ ou $y=0$.

Definição. Seja $R$ uma álgebra prima e seja $U$ uma subálgebra de $R$. Dizemos que $U$ é racionalmente completa se a seguinte condição for satisfeita:

Seja $a \in R$ e seja $I$ um ideal não nulo de $U$. Se $a I \subseteq U$, então $a \in U$.

É imediato verificar que se $U$ é racionalmente completa e contém um ideal não nulo de $R$, então $U=R$.

A seguir temos uma série de notações e definições que serão utilizadas ao longo deste trabalho:

- Seja $R$ uma álgebra prima. Vamos denotar por $\mathcal{F}(R)$ o conjunto de todos ideais não nulos de $R$.

- Seja $A$ um espaço vetorial, seja $\nu \in A$ e seja $n$ um inteiro não negativo. Denotamos

$$
n \nu=\underbrace{\nu+\cdots+\nu}_{n \text { vezes }}
$$

Se $A$ for uma álgebra, denotamos

$$
\nu^{0}=1_{A} \quad \text { e } \quad \nu^{n}=\nu \nu^{n-1}, \text { para } n \geq 1 .
$$

- Seja $A$ uma álgebra e seja $M$ um $A$-módulo à esquerda. Denotamos a ação de $A$ em $M$ por $a \cdot m \in M$, para todo $a \in A$ e todo $m \in M$, a menos que especificado de outra maneira.

- Seja $A$ uma álgebra e seja $M$ um $A$-módulo à esquerda. Dizemos que $M$ é um $A$-módulo à esquerda injetivo se, para qualquer monomorfismo de $A$-módulos à esquerda $f: X \longrightarrow Y$ e qualquer homomorfismo de $A$-módulos à esquerda $g: X \longrightarrow M$, existir um homomorfismo de 
$A$-módulos à esquerda $h: Y \longrightarrow M$ tal que $g=h \circ f$. Define-se de forma análoga o conceito de $A$-módulo à direita injetivo.

- Seja $A$ uma álgebra e seja $M$ um $A$-módulo à esquerda. Dizemos que $M$ é um $A$-módulo à esquerda artiniano se os submódulos de $M$ satisfizerem a condição de cadeia descendente, ou seja, não existir uma cadeia infinita $M \supset M_{1} \supset M_{2} \supset \cdots$, de submódulos de $M$ distintos dois a dois. Define-se de forma análoga o conceito de $A$-módulo à direita artiniano.

- Seja $A$ uma álgebra e sejam $V, W$ dois subconjuntos não vazios de $A$. Vamos denotar

$$
V^{W}=\{v \in V: v w=w v, \text { para todo } w \in W\}
$$

o centralizador de $W$ em $V$.

- Seja $V$ um espaço vetorial e sejam $m_{1}, \ldots, m_{n}$ elementos de $V$. Denotamos por $\operatorname{span}_{k}\left\{m_{i}\right\}_{1 \leq i \leq n}$ o subespaço de $V$ gerado por $m_{1}, \ldots, m_{n}$. Ou seja,

$$
\operatorname{span}_{k}\left\{m_{i}\right\}_{1 \leq i \leq n}=\left\{\lambda_{1} m_{1}+\cdots+\lambda_{n} m_{n}: \lambda_{1}, \ldots, \lambda_{n} \in k\right\}
$$

- Sejam $V$ e $W$ espaços vetoriais. A aplicação linear $\tau: V \otimes W \longrightarrow W \otimes V$ tal que $\tau(v \otimes w)=w \otimes v$, para todo $v \in V$ e todo $w \in W$, é denominada aplicação twist.

- Sejam $V$ e $W$ espaços vetoriais. Denotamos por $\operatorname{hom}_{k}(V, W)$ o conjunto das transformações lineares de $V$ em $W$.

- Sejam $U, V$ e $W$ espaços vetoriais, e sejam $u \in U$ e $v \in V$. Dizemos que uma forma bilinear $B: U \times V \longrightarrow W$ é não degenerada se $B(U, v)=0$ implicar $v=0$, e se $B(u, V)=0$ implicar $u=0$.

- Sejam $V$ e $W$ espaços vetoriais. Denotamos por $V^{*}=\operatorname{hom}_{k}(V, k)$ o espaço dual de $V$. Temos que $V$ e $V^{*}$ determinam uma forma bilinear não degenerada

$$
\begin{array}{rlc}
\langle,\rangle: V^{*} \times V & \longrightarrow & k \\
(f, v) & \longmapsto\langle f, v\rangle=f(v)
\end{array}
$$

Se $\phi: V \longrightarrow W$ é uma aplicação linear, então a transposta de $\phi$ é a aplicação linear $\phi^{*}: W^{*} \longrightarrow$ $V^{*}$ definida por $\phi^{*}(f)(v)=f(\phi(v))$, para todo $f \in W^{*}$ e todo $v \in V$. Note também que 
podemos escrever $\left\langle\phi^{*}(f), v\right\rangle=\langle f, \phi(v)\rangle$.

- Seja $G$ um grupo. Dizemos que uma álgebra $A$ é $G$-graduada se existir uma família $\left\{A_{g}\right\}_{g \in G}$ de subespaços de $A$ indexada por $G$ tal que $A=\bigoplus_{g \in G} A_{g}, 1_{A} \in A_{1_{G}}$, onde $1_{G}$ denota o elemento neutro de $G$, e $A_{g} A_{h} \subseteq A_{g h}$, para todos $g, h \in G$. Dizemos que a álgebra $A$ é fortemente graduada por $G$ se $A_{g} A_{h}=A_{g h}$, para todos $g, h \in G$.

Caso $A$ seja $G$-graduada, denotamos por $a_{g}$ a componente de $a$ em $A_{g}$, para todo $a \in A$ e todo $g \in G$. Assim, podemos escrever $a=\sum_{g \in G} a_{g}$, para todo $a \in A$.

- Seja $G$ um grupo e seja $k G$ o espaço vetorial de base $G$, isto é,

$$
k G=\left\{\sum_{g \in G} \lambda_{g} g: \lambda_{g} \in k, \text { para todo } g \in G\right\}
$$

O espaço vetorial $k G$ é uma álgebra com multiplicação induzida por

$$
\begin{gathered}
k G \times k G \quad \longrightarrow \quad k G \\
\left(\lambda_{g} g, \lambda_{h} h\right) \quad \longmapsto \lambda_{g} \lambda_{h} g h .
\end{gathered}
$$

Observe que a unidade da álgebra $k G$ é $1_{k} 1_{G}$, onde $1_{G}$ denota o elemento neutro de $G$. A álgebra $k G$ é denominada álgebra de grupo do grupo $G$.

\section{3 Álgebras de Hopf}

O objetivo desta seção é definir a estrutura denominada álgebra de Hopf e apresentar diversas propriedades e resultados que terão algum uso no decorrer deste trabalho. Convém ressaltar que mais detalhes sobre as definições e resultados aqui expostos podem ser encontrados em [FM06, Swe69, DNR00, Mon93b].

\subsubsection{Definição}

Inicialmente, com o objetivo de definir apropriadamente as álgebras de Hopf, vamos expressar a definição de álgebra feita anteriormente através de diagramas comutativos. 
Definição. Seja $A$ um espaço vetorial e sejam $m: A \otimes A \longrightarrow A$ e $\mu: k \longrightarrow A$ aplicações lineares. Dizemos que a terna ordenada $(A, m, \mu)$ é uma álgebra se os seguintes diagramas forem comutativos:
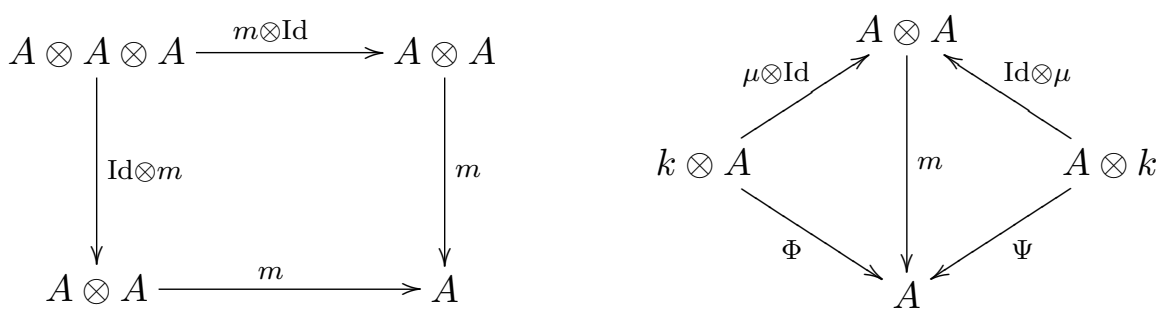

Note que $\Phi$ e $\Psi$ são os isomorfismos canônicos entre os espaços vetoriais envolvidos, ou seja, $\Phi(\lambda \otimes a)=\Psi(a \otimes \lambda)=\lambda a$, para todo $\lambda \in k$ e todo $a \in A$. Denominamos $m$ a multiplicação e $\mu$ a aplicação unidade de $A$. O primeiro diagrama representa a associatividade da multiplicação e o segundo representa a existência de elemento unidade em $A$. Note que $\mu\left(1_{k}\right)=1_{A}$.

A partir de agora, sempre que tivermos uma estrutura de álgebra, $m$ será a sua multiplicação e $\mu$ será a sua aplicação unidade, a menos de menção explícita ao contrário. No decorrer desta seção, $A$ representará uma álgebra.

Ao dualizar a definição de álgebra dada acima, obtemos a estrutura denominada coálgebra.

Definição. Seja $C$ um espaço vetorial e sejam $\Delta: C \longrightarrow C \otimes C$ e $\varepsilon: C \longrightarrow k$ aplicações lineares. Dizemos que a terna ordenada $(C, \Delta, \varepsilon)$ é uma coálgebra se os seguintes diagramas forem comutativos:
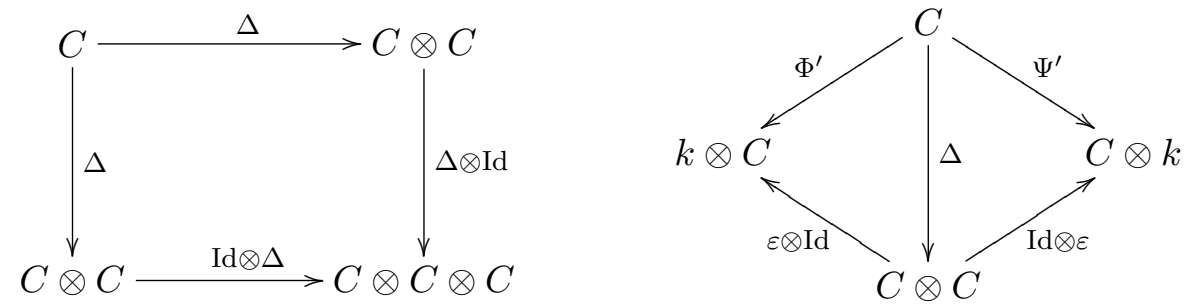

Note que $\Phi^{\prime}$ e $\Psi^{\prime}$ são os isomorfismos canônicos entre os espaços vetoriais envolvidos, ou seja, $\Phi^{\prime}(c)=1_{k} \otimes c$ e $\Psi^{\prime}(c)=c \otimes 1_{k}$, para todo $c \in C$. Denominamos $\Delta$ a comultiplicação e $\varepsilon$ a counidade de $C$. O primeiro diagrama representa a chamada coassociatividade da comultiplicação.

A partir de agora, sempre que tivermos uma estrutura de coálgebra, $\Delta$ será a sua comultiplicação e $\varepsilon$ será a sua counidade, a menos de menção explícita ao contrário. No decorrer desta seção, $C$ representará uma coálgebra. 
Observação. (i) O segundo diagrama da definição de coálgebras implica na injetividade da comultiplicação.

(ii) Denotamos $\Delta(c)=\sum_{(c)} c_{(1)} \otimes c_{(2)}$, para todo $c \in C$. Essa notação é chamada notação sigma. Ao longo do texto, muitas vezes omitimos o índice do somatório com a intenção de não carregar muito a notação. Note que, pelo segundo diagrama da definição de coálgebras, temos que

$$
\sum_{(c)} \varepsilon\left(c_{(1)}\right) c_{(2)}=\sum_{(c)} \varepsilon\left(c_{(2)}\right) c_{(1)}=c
$$

(ii’) Da coassociatividade da comultiplicação, denotamos

$$
\Delta_{2}(c)=\sum_{(c)} c_{(1)} \otimes c_{(2)} \otimes c_{(3)}=\sum_{(c)} \sum_{\left(c_{(1)}\right)} c_{(1)_{(1)}} \otimes c_{(1)_{(2)}} \otimes c_{(2)}=\sum_{(c)} \sum_{\left(c_{(2)}\right)} c_{(1)} \otimes c_{(2)_{(1)}} \otimes c_{(2)_{(2)}} .
$$

(iii) Seja $J$ um subconjunto qualquer de $C$. Denotamos $J^{+}=J \cap \operatorname{ker}(\varepsilon)$.

Para qualquer coálgebra $C$, podemos definir a sua coálgebra co-oposta $C^{\text {cop }}$, que coincide com $C$ como espaço vetorial, cuja counidade coincide $\operatorname{com} \varepsilon$, e cuja comultiplicação, chamada comultiplicação co-oposta, é dada por $\Delta^{\text {cop }}=\tau \circ \Delta$. Observe que uma álgebra $A$ é comutativa se $m \circ \tau=m$ em $A \otimes A$. Da mesma forma, dizemos que $C$ é cocomutativa se $\tau \circ \Delta=\Delta$ em $C$.

Sejam $\left(C, \Delta_{C}, \varepsilon_{C}\right)$ e $\left(D, \Delta_{D}, \varepsilon_{D}\right)$ coálgebras. Dizemos que uma aplicação linear $f: C \longrightarrow D$ é um morfismo de coálgebras se $\Delta_{D} \circ f=(f \otimes f) \circ \Delta_{C}$ e $\varepsilon_{C}=\varepsilon_{D} \circ f$.

Definição. Seja $I$ um subespaço de $C$. Dizemos que:

(i) $I$ é um coideal se $\Delta(I) \subseteq I \otimes C+C \otimes I$ e $\varepsilon(I)=0$.

(ii) $I$ é um coideal à direita se $\Delta(I) \subseteq I \otimes C$.

(iii) $I$ é um coideal à esquerda se $\Delta(I) \subseteq C \otimes I$.

(iv) $I$ é uma subcoálgebra se $\Delta(I) \subseteq I \otimes I$.

Em qualquer coálgebra existem alguns tipos de elementos que são bem importantes, e por isso mesmo até recebem um nome. 
Definição. Seja $c \in C$.

(i) Dizemos que $c$ é group-like se $\Delta(c)=c \otimes c$ e $\varepsilon(c)=1$. O conjunto dos elementos group-like de $C$ é denotado por $G(C)$.

(ii) Dizemos que $c$ é $g$, $h$-primitivo se $\Delta(c)=g \otimes c+c \otimes h$, onde $g, h \in G(C)$. O conjunto dos elementos $g$, h-primitivos é denotado por $P_{g, h}(C)$.

Observação. Por [Swe69, Proposition 3.2.1], temos que elementos group-like distintos são linearmente independentes. Além disso, sejam $g, h \in G(C)$. É fácil ver que $k(g-h) \subseteq P_{g, h}(C)$. Então, definimos $P_{g, h}^{\prime}(C)$ como um subespaço de $P_{g, h}(C)$ complementar a $k(g-h)$.

Segue um importante resultado sobre a relação entre o conjunto dos elementos group-like e os elementos primitivos, em uma coálgebra qualquer.

Proposição 1.3.1. Sejam $g, h$ elementos distintos de $G(C)$. Então:

(i) $P_{g, g}(C) \cap k G(C)=\{0\}$.

(ii) $P_{g, h}(C) \cap k G(C)=k(g-h)$.

Demonstração. Antes de mais nada, lembre que elementos group-like distintos são linearmente independentes.

(i) Seja $x \in P_{g, g}(C) \cap k G(C)$. Podemos escrever $x=\sum_{\sigma \in G(C)} \lambda_{\sigma} \sigma$, onde $\lambda_{\sigma} \in k$. Temos então:

$$
\Delta(x)=g \otimes x+x \otimes g=\sum_{\sigma \in G(C)} \lambda_{\sigma}(g \otimes \sigma)+\sum_{\sigma \in G(C)} \lambda_{\sigma}(\sigma \otimes g) .
$$

Por outro lado,

$$
\Delta(x)=\Delta\left(\sum_{\sigma \in G(C)} \lambda_{\sigma} \sigma\right)=\sum_{\sigma \in G(C)} \lambda_{\sigma}(\sigma \otimes \sigma) .
$$

Igualando as duas expressões acima, é fácil ver que $\lambda_{\sigma}=0$, para todo $\sigma \in G(C)$ com $\sigma \neq g$. Daí, temos

$$
\left(\lambda_{g}+\lambda_{g}\right)(g \otimes g)=\lambda_{g}(g \otimes g)
$$


Da expressão acima, $\lambda_{g}=0$. Logo, $x=0$.

(ii) Seja $x \in P_{g, h}(C) \cap k G(C)$. Podemos escrever $x=\sum_{\sigma \in G(C)} \lambda_{\sigma} \sigma$, onde $\lambda_{\sigma} \in k$. Temos então:

$$
\Delta(x)=g \otimes x+x \otimes h=\sum_{\sigma \in G(C)} \lambda_{\sigma}(g \otimes \sigma)+\sum_{\sigma \in G(C)} \lambda_{\sigma}(\sigma \otimes h) .
$$

Por outro lado,

$$
\Delta(x)=\Delta\left(\sum_{\sigma \in G(C)} \lambda_{\sigma} \sigma\right)=\sum_{\sigma \in G(C)} \lambda_{\sigma}(\sigma \otimes \sigma) .
$$

Igualando as duas expressões acima, é fácil ver que $\lambda_{\sigma}=0$, para todo $\sigma \in G(C) \operatorname{com} \sigma \notin\{g, h\}$. Daí, temos

$$
\lambda_{g}(g \otimes g)+\lambda_{h}(g \otimes h)+\lambda_{g}(g \otimes h)+\lambda_{h}(h \otimes h)=\lambda_{g}(g \otimes g)+\lambda_{h}(h \otimes h) .
$$

Da expressão acima, temos que $\lambda=\lambda_{g}=-\lambda_{h}$. Assim, $x=\lambda(g-h)$. Além disso, todo elemento da forma $\lambda(g-h)$, onde $\lambda \in k$, claramente pertence à intersecção. Logo, podemos concluir que $P_{g, h}(C) \cap k G(C)=k(g-h)$.

Combinando as noções de álgebra e coálgebra temos a seguinte estrutura.

Definição. Seja $B$ um espaço vetorial. Dizemos que $B$ é uma biálgebra se $(B, m, \mu)$ for uma álgebra, se $(B, \Delta, \varepsilon)$ for uma coálgebra e se as seguintes condições forem satisfeitas:

(1) $\Delta$ e $\varepsilon$ são morfismos de álgebras;

(2) $m$ e $\mu$ são morfismos de coálgebras.

Observação. Por [Swe69, Proposition 3.1.1], temos que as condições acima são equivalentes.

Seja $B$ uma biálgebra e sejam $g, h \in G(B)$. Note que $\Delta(g h)=\Delta(g) \Delta(h)=(g \otimes g)(h \otimes h)=$ $g h \otimes g h$, e que $\varepsilon(g h)=\varepsilon(g) \varepsilon(h)=1_{k} 1_{k}=1_{k}$, ou seja, $g h \in G(B)$. Como $B$ possui estrutura de álgebra, existe elemento unidade $1_{B} \in B$. Do fato da comultiplicação e da counidade de $B$ serem morfismos de álgebras, temos que $1_{B} \in G(B)$. Assim, o conjunto dos elementos group-like de uma biálgebra formam um monóide. 
Como $1 \in G(B)$, existe o conjunto dos elementos 1, 1-primitivos. Denotamos $P_{1,1}(B)=P(B)$ e denominamos seus elementos de elementos primitivos de $B$. Segue um resultado sobre os elementos primitivos de uma biálgebra.

Proposição 1.3.2. Seja $B$ uma biálgebra e seja $x \in P(B)$. Então:

(i) Seja n um inteiro não-negativo. Temos que

$$
\Delta\left(x^{n}\right)=\sum_{i=0}^{n}\left(\begin{array}{l}
n \\
i
\end{array}\right) x^{i} \otimes x^{n-i}
$$

onde $\left(\begin{array}{l}n \\ i\end{array}\right)=\frac{n !}{i !(n-i) !}$ é o coeficiente binomial, para todo $0 \leq i \leq n$.

(ii) Se o corpo base $k$ tiver característica zero e se $B$ tiver dimensão finita, então $x=0$.

Demonstração. (i) Seja $A$ uma álgebra qualquer e sejam $a, b \in A$. Pelo desenvolvimento do binômio de Newton, temos

$$
(a+b)^{n}=\sum_{i=0}^{n}\left(\begin{array}{c}
n \\
i
\end{array}\right) a^{n-i} b^{i}
$$

Assim, temos que

$$
\Delta\left(x^{n}\right)=(\Delta(x))^{n}=\left(1_{B} \otimes x+x \otimes 1_{B}\right)^{n}=\sum_{i=0}^{n}\left(\begin{array}{c}
n \\
i
\end{array}\right)\left(1_{B} \otimes x\right)^{n-i}\left(x \otimes 1_{B}\right)^{i}=\sum_{i=0}^{n}\left(\begin{array}{c}
n \\
i
\end{array}\right) x^{i} \otimes x^{n-i} .
$$

(ii) Vamos supor que $x \neq 0$. Vamos mostrar por indução em $n$ que $\left\{1_{B}, x, \ldots, x^{n}\right\}$ é linearmente independente.

Vamos mostrar que vale para $n=1$. Supondo que $\left\{1_{B}, x\right\}$ é linearmente dependente, podemos escrever $x=\lambda 1_{B}$, onde $\lambda \in k$. Assim, temos que

$$
\Delta(x)=1_{B} \otimes x+x \otimes 1_{B}=(\lambda+\lambda)\left(1_{B} \otimes 1_{B}\right) .
$$

Por outro lado,

$$
\Delta(x)=\Delta\left(\lambda 1_{B}\right)=\lambda\left(1_{B} \otimes 1_{B}\right)
$$


Das expressões acima, temos que $\lambda+\lambda=\lambda$, ou seja, $\lambda=0$. Logo, concluímos que $x=0$, o que é um absurdo.

Supondo que $\left\{1_{B}, x, \ldots, x^{n}\right\}$ é linearmente independente, vamos mostrar que $\left\{1_{B}, x, \ldots, x^{n}, x^{n+1}\right\}$ é linearmente independente. Podemos supor que $x^{n+1}=\sum_{j=0}^{n} \lambda_{j} x^{j}$, onde $\lambda_{j} \in k$, para todo $j$. De (i), temos que

$$
\begin{aligned}
\Delta\left(x^{n+1}\right) & =\sum_{i=0}^{n+1}\left(\begin{array}{c}
n+1 \\
i
\end{array}\right) x^{i} \otimes x^{n+1-i} \\
& =1_{B} \otimes x^{n+1}+(n+1)\left(x \otimes x^{n}\right)+\sum_{i=2}^{n+1}\left(\begin{array}{c}
n+1 \\
i
\end{array}\right) x^{i} \otimes x^{n+1-i} .
\end{aligned}
$$

Pelo fato da característica do corpo base ser zero, e $x$ e $x^{n}$ serem não nulos, temos que o termo $(n+1)\left(x \otimes x^{n}\right)$ é não nulo na expressão acima. Mas,

$$
\Delta\left(x^{n+1}\right)=\Delta\left(\sum_{j=0}^{n} \lambda_{j} x^{j}\right)=\sum_{j=0}^{n}\left[\sum_{i=0}^{j} \lambda_{j}\left(\begin{array}{l}
j \\
i
\end{array}\right) x^{i} \otimes x^{j-i}\right]
$$

não possui nenhuma componente no subespaço $k\left(x \otimes x^{n}\right)$.

Pela hipótese indutiva, $\left\{1_{B}, x, \ldots, x^{n}\right\}$ é linearmente independente. Logo, não pode haver igualdade, o que é um absurdo.

Dessa forma, $\left\{1_{B}, x, \ldots, x^{n}, x^{n+1}\right\}$ é linearmente independente. Como a dimensão de $B$ é finita, chegamos em uma contradição. Portanto, $x=0$.

Sejam $B$ e $B^{\prime}$ biálgebras. Dizemos que uma aplicação linear $f: B \longrightarrow B^{\prime}$ é um morfismo de biálgebras se $f$ for um morfismo de álgebras e um morfismo de coálgebras. Seja $I$ um subespaço de $B$. Se $I$ for um ideal e um coideal dizemos que $I$ é um bi-ideal.

Sejam $f, g \in \operatorname{hom}_{k}(C, A)$. Note que podemos definir uma estrutura de álgebra em $\operatorname{hom}_{k}(C, A)$ com a seguinte multiplicação, denominada produto de convolução:

$$
(f * g)(c)=(m \circ(f \otimes g) \circ \Delta)(c)=\sum_{(c)} f\left(c_{(1)}\right) g\left(c_{(2)}\right), \text { para todo } c \in C
$$


Observe que a aplicação composta $\mu \circ \varepsilon \in \operatorname{hom}_{k}(C, A)$ é a unidade da álgebra $\operatorname{hom}_{k}(C, A)$ com o produto de convolução. Finalmente, estamos em condições de definir a estrutura denominada álgebra de Hopf.

Definição. Seja $H$ uma biálgebra. Dizemos que $H$ é uma álgebra de Hopf se a identidade de $H$ for inversível no produto de convolução em $\operatorname{hom}_{k}(H, H)$. A inversa de $\operatorname{Id}_{H}$ no produto de convolução é chamada de antípoda e é denotada por $S$.

Observação. Na notação sigma,

$$
\sum_{(h)} S\left(h_{(1)}\right) h_{(2)}=\sum_{(h)} h_{(1)} S\left(h_{(2)}\right)=(\mu \circ \varepsilon)(h)=\varepsilon(h) 1_{H}
$$

Além disso, por [Swe69, Proposition 4.0.1], temos:

(i) $S$ é um antimorfismo de álgebras, ou seja, $S(g h)=S(h) S(g)$, para todos $g, h \in H$, e $S(1)=1$.

(ii) $S$ é um antimorfismo de coálgebras, ou seja, $\Delta \circ S=\tau \circ(S \otimes S) \circ \Delta$ e $\varepsilon \circ S=\varepsilon$. Na notação sigma,

$$
\sum_{(S(h))} S(h)_{(1)} \otimes S(h)_{(2)}=\sum_{(h)} S\left(h_{(2)}\right) \otimes S\left(h_{(1)}\right) .
$$

Exemplo 1.3.3. Seja $G$ um grupo e seja $H=k G$ sua álgebra de grupo. Se definirmos $\Delta(g)=g \otimes g$, $\varepsilon(g)=1$ e $S(g)=g^{-1}$, para todo $g \in G$, então $H$ é uma álgebra de Hopf.

A partir de agora, sempre que tivermos uma estrutura de álgebra de Hopf, $S$ será a sua antípoda, a menos de menção explícita ao contrário. Caso $S$ possua uma inversa na composição, ela será denotada por $\bar{S}$. No decorrer desta seção, $H$ representará uma álgebra de Hopf.

Seja $g \in G(H)$. Note que $S(g) g=g S(g)=\varepsilon(g) 1=1$, ou seja, $g$ possui inversa $g^{-1}=S(g)$. Além disso, do fato de $S$ ser um antimorfismo de coálgebras, temos que $\Delta(S(g))=S(g) \otimes S(g)$. Portanto, $S(g)$ é um elemento group-like e o conjunto $G(H)$ é um grupo.

Sejam $H$ e $H^{\prime}$ álgebras de Hopf. Dizemos que uma aplicação linear $f: H \longrightarrow H^{\prime}$ é um morfismo de álgebras de Hopf se $f$ for um morfismo de biálgebras. Seja $I$ um subespaço de $H$. Se $I$ for um bi-ideal e se $S(I) \subseteq I$, dizemos que $I$ é um ideal de Hopf. Nesse caso, o quociente $H / I$ é uma álgebra de Hopf com a estrutura induzida de $H$, e a projeção de $H$ em $H / I$ é um morfismo de álgebras de Hopf. 
Proposição 1.3.4. Seja $H$ uma álgebra de Hopf. Então $\operatorname{ker}(\varepsilon)$ é um ideal de Hopf de H.

Demonstração. Como $\varepsilon$ é um morfismo de álgebras, é imediato que ker( $(\varepsilon)$ é um ideal de $H$. Além disso, do fato de $S$ ser um antimorfismo de coálgebras, temos que $\varepsilon \circ S=\varepsilon$. Daí, é imediato que $S(\operatorname{ker}(\varepsilon)) \subseteq \operatorname{ker}(\varepsilon)$. Resta mostrar que $\operatorname{ker}(\varepsilon)$ é um coideal de $H$.

Como $\operatorname{ker}(\varepsilon)$ tem codimensão igual a um e $\varepsilon\left(1_{H}\right)=1_{k}$, pois $\varepsilon$ é um morfismo de álgebras, podemos obter $\left\{h_{i}\right\} \cup\left\{1_{H}\right\}$ base de $H$ tal que $\left\{h_{i}\right\}$ é base de $\operatorname{ker}(\varepsilon)$. Seja $h \in\left\{h_{i}\right\}$. Podemos escrever

$$
\Delta(h)=a \otimes 1_{H}+\sum_{i} a_{i} \otimes h_{i} \text {, onde } a, a_{i} \in H, \text { para todo } i
$$

Assim, temos que $h=a \varepsilon\left(1_{H}\right)+\sum_{i} a_{i} \varepsilon\left(h_{i}\right)=a$ e, portanto, $a \in \operatorname{ker}(\varepsilon)$, ou seja, $\Delta(h) \in \operatorname{ker}(\varepsilon) \otimes$ $H+H \otimes \operatorname{ker}(\varepsilon)$. Como $h$ é um elemento qualquer da base de $\operatorname{ker}(\varepsilon)$, concluímos que $\operatorname{ker}(\varepsilon)$ é um coideal de $H$.

Definição. Seja $I$ um subespaço de $H$. Dizemos que $I$ é uma subálgebra de Hopf de $H$ se $I$ for uma subálgebra e uma subcoálgebra de $H$, e se $S(I) \subseteq I$.

Definição. Seja $I$ um subespaço de $H$. Dizemos que $I$ é um coideal subálgebra à direita se $I$ for subálgebra e coideal à direita de $H$. Define-se analogamente coideal subálgebra à esquerda.

Vamos denotar por $H^{\text {cop }}$ a biálgebra com comultiplicação co-oposta. Então, temos o seguinte resultado extraído de [Mon93b, Lemma 1.5.11].

Proposição 1.3.5. H é uma álgebra de Hopf com antípoda inversível na composição se, e somente se, $H^{\mathrm{cop}}$ for uma álgebra de Hopf com antípoda inversível na composição. Neste caso, $\bar{S}$ é a antípoda de $H^{\mathrm{cop}}$.

Observação. Note que $\bar{S}$ é também um antimorfismo de álgebras e um antimorfismo de coálgebras. Além disso, $\bar{S}$ é a inversa de $\operatorname{Id}_{H^{\text {cop }}}$ no produto de convolução. Na notação sigma,

$$
\sum_{(h)} \bar{S}\left(h_{(2)}\right) h_{(1)}=\sum_{(h)} h_{(2)} \bar{S}\left(h_{(1)}\right)=(\mu \circ \varepsilon)(h)=\varepsilon(h) 1_{H}
$$




\subsubsection{Ações, produto cruzado e o produto smash}

Inicialmente, vamos definir o conceito de ação de uma álgebra de Hopf em uma álgebra qualquer. Em seguida definiremos a estrutura conhecida como produto cruzado, e depois veremos que o produto smash de álgebras é um caso particular dessa estrutura.

Definição. Dizemos que $H$ age em $A$ ou que $A$ é um $H$-módulo álgebra à esquerda se as seguintes condições forem satisfeitas:

(i) $A$ é um $H$-módulo à esquerda.

(ii) $h \cdot(a b)=\sum_{(h)}\left(h_{(1)} \cdot a\right)\left(h_{(2)} \cdot b\right)$, para todo $h \in H$ e todos $a, b \in A$.

(iii) $h \cdot 1_{A}=\varepsilon(h) 1_{A}$, para todo $h \in H$.

Dizemos que $H$ mede $A$ se (ii) e (iii) forem satisfeitos.

Seja $A$ um $H$-módulo álgebra à esquerda, e sejam $h \in H$ e $a \in A$. Dizemos que $h$ age trivialmente em $a$ se $h \cdot a=\varepsilon(h) a$. O conjunto

$$
A^{H}=\{a \in A: h \cdot a=\varepsilon(h) a, \text { para todo } h \in H\}
$$

é uma subálgebra de $A$ denominada a subálgebra de invariantes de $A$ sob a ação de $H$.

Definição. Podemos definir as seguintes ações de $H$ em $H$ :

(i) A ação adjunta à esquerda de $H$ em $H$ é definida por $\operatorname{ad}_{1}(h, b)=\sum h_{(1)} b S\left(h_{(2)}\right)$, para todos $b, h \in H$.

(ii) A ação adjunta à direita de $H$ em $H$ é definida por $\operatorname{ad}_{\mathrm{r}}(h, b)=\sum S\left(h_{(1)}\right) b h_{(2)}$, para todos $b, h \in H$.

É fácil verificar que ambas as ações adjuntas definem de fato ações de $H$ em $H$.

Definição. Seja $I$ uma subálgebra de Hopf de $H$. Dizemos que $I$ é uma subálgebra de Hopf normal se $\operatorname{ad}_{\mathrm{l}}(H, I) \subseteq I$ e $\operatorname{ad}_{\mathrm{r}}(H, I) \subseteq I$. 
Segue um lema extraído de [Mon93b, Lemma 3.4.2] sobre subálgebras de Hopf normais.

Lema 1.3.6. Se $K$ uma subálgebra de Hopf normal de $H$, então $I=H K^{+}=K^{+} H$ é um ideal de Hopf.

Demonstração. Sejam $h \in H$ e $a \in K$. Considere a seguinte identidade

$$
h a=\sum h_{(1)} a \varepsilon\left(h_{(2)}\right) 1_{H}=\sum h_{(1)} a S\left(h_{(2)}\right) h_{(3)}=\sum \operatorname{ad}_{1}\left(h_{(1)}, a\right) h_{(2)} .
$$

A partir da identidade acima e do fato de $K$ ser uma subálgebra de Hopf normal de $H$, temos que $h a \in K H$. Além disso, se $\varepsilon(a)=0$, é fácil ver que $\varepsilon\left(\operatorname{ad}_{l}(h, a)\right)=0$. Logo, $H K^{+} \subseteq K^{+} H$.

Analogamente, só que utilizando $\operatorname{ad}_{\mathrm{r}}(h, a)$ ao invés de $\operatorname{ad}_{l}(h, a)$, temos que $K^{+} H \subseteq H K^{+}$. Assim, mostramos que $I=H K^{+}=K^{+} H$. A partir daí, é fácil ver que $I$ é um ideal de $H$.

A partir da Proposição 1.3.4, temos que

$$
\Delta\left(K^{+}\right)=\Delta(K \cap \operatorname{ker}(\varepsilon)) \subseteq(K \otimes K) \cap(\operatorname{ker}(\varepsilon) \otimes H+H \otimes \operatorname{ker}(\varepsilon))=K^{+} \otimes K+K \otimes K^{+} .
$$

Da expressão acima e do fato de $\varepsilon(I)=\varepsilon\left(K^{+}\right) \varepsilon(H)=0$, concluímos que $I$ é um coideal de $H$. Falta mostrar que $S(I) \subseteq I$.

Novamente a partir da Proposição 1.3.4, sabemos que $\operatorname{ker}(\varepsilon)$ é um ideal de Hopf. Assim,

$$
S\left(K^{+}\right)=S(K \cap \operatorname{ker}(\varepsilon)) \subseteq(S(K) \cap S(\operatorname{ker}(\varepsilon))) \subseteq K \cap \operatorname{ker}(\varepsilon)=K^{+} .
$$

Logo, temos que $S(I)=S\left(K^{+} H\right)=S(H) S\left(K^{+}\right) \subseteq H K^{+}=I$ e, portanto, $I$ é um ideal de Hopf.

Observação. Lembre que, conforme vimos na seção anterior, o quociente $H / I$ é uma álgebra de Hopf com a estrutura induzida de $H$, e a projeção de $H$ em $H / I$ é um morfismo de álgebras de Hopf.

Agora vamos definir a estrutura denominada produto smash. Seja $H$ uma álgebra de Hopf que age em $A$. Denotamos por $A \# H$ a álgebra que coincide com $A \otimes H$ como espaço vetorial e cuja 
multiplicação é dada por

$$
(a \# h)(b \# g)=\sum_{(h)} a\left(h_{(1)} \cdot b\right) \# h_{(2)} g, \text { para todos } a, b \in A \text { e todos } h, g \in H .
$$

Observe que $a \# h$ e $b \# g$ denotam respectivamente os elementos $a \otimes h$ e $b \otimes g$ de $A \# H$. Denominamos $A \# H$ o produto smash de $A$ e $H$.

Observação. É fácil verificar que $A \# H$ é de fato uma álgebra com a multiplicação definida acima. Note também que $1_{A} \# 1_{H}$ é o elemento unidade de $A \# H$.

Observe que através das inclusões

$$
\begin{aligned}
& A \longrightarrow A \# H \quad H \longrightarrow A \# H \\
& a \longmapsto a \# 1_{H} \quad h \longmapsto 1_{A} \# h
\end{aligned}
$$

podemos identificar $A$ e $H$ com as subálgebras $A \# 1_{H}$ e $1_{A} \# H$ de $A \# H$, respectivamente. Assim, $A \# H$ tem uma estrutura de $A$-módulo, tanto à direita quanto à esquerda. Da mesma forma $A \# H$ tem uma estrutura de $H$-módulo, tanto à direita quanto à esquerda.

Note também que $A$ tem estrutura de $A \# H$-módulo à esquerda se definirmos

$$
(a \# h) \cdot x=a(h \cdot x) \text {, para todos } a, x \in A \text { e todo } h \in H \text {. }
$$

Caso a antípoda de $H$ seja bijetora, $A$ também tem estrutura de $A \# H$-módulo à direita se definirmos

$$
x \triangleleft(a \# h)=\bar{S}(h) \cdot(x a), \text { para todos } a, x \in A \text { e todo } h \in H .
$$

Seja $\sigma: H \times H \longrightarrow A$ uma função $k$-bilinear. Dizemos que $\sigma$ é inversivel no produto de convolução se existir uma função $k$-bilinear $\sigma^{-1}: H \times H \longrightarrow A$ tal que $\sigma * \sigma^{-1}=\sigma^{-1} * \sigma=\varepsilon_{H \times H}$

$$
\begin{aligned}
\left(\sigma * \sigma^{-1}\right)(l, m) & =\sum \sigma\left(l_{(1)}, m_{(1)}\right) \sigma^{-1}\left(l_{(2)}, m_{(2)}\right) \\
& =\varepsilon(l) \varepsilon(m) 1_{A} \\
& =\sum \sigma^{-1}\left(l_{(1)}, m_{(1)}\right) \sigma\left(l_{(2)}, m_{(2)}\right) \\
& =\left(\sigma^{-1} * \sigma\right)(l, m), \text { para todos } l, m \in H .
\end{aligned}
$$


Observe que a função $k$-bilinear

$$
\begin{array}{ccc}
\varepsilon_{H \times H}: \quad H \times H & \longrightarrow & A \\
& (l, h) & \longmapsto \varepsilon(l) \varepsilon(m) 1_{A}
\end{array}
$$

é a identidade do produto de convolução na álgebra das funções $k$-bilineares de $H \times H$ em $A$.

Agora estamos em condições de definir a estrutura conhecida como produto cruzado, que será utilizada no caminho para se demonstrar a sobrejeção da correspondência.

Definição. Assuma que $H$ mede $A$ e que $\sigma: H \times H \longrightarrow A$ é uma função $k$-bilinear inversível no produto de convolução. Denotamos por $A \#_{\sigma} H$ o $k$-espaço vetorial $A \otimes H$ com multiplicação dada por

$$
\left(a \#_{\sigma} h\right)\left(b \#_{\sigma} l\right)=\sum a\left(h_{(1)} \cdot b\right) \sigma\left(h_{(2)}, l_{(1)}\right) \#_{\sigma} h_{(3)} l_{(2)}, \text { para todos } h, l \in H \text { e todos } a, b \in A
$$

Observe que $a \#_{\sigma} h$ e $b \#_{\sigma} l$ denotam respectivamente os elementos $a \otimes h$ e $b \otimes l$ de $A \#_{\sigma} H$. O objeto $A \#_{\sigma} H$ é chamado produto cruzado se a multiplicação for associativa e $1_{A} \#_{\sigma} 1_{H}$ for o elemento unidade de $A \#_{\sigma} H$, ou seja, se $A \#_{\sigma} H$ for uma álgebra.

O próximo lema, extraído de [Mon93b, Lemma 7.1.2], traz as condições que devem ser satisfeitas para que a estrutura construída acima seja um produto cruzado.

Lema 1.3.7. Sejam H, A e $\sigma$ como na definição acima. Então $A \#_{\sigma} H$ é um produto cruzado se, $e$ somente se, as seguintes condições forem satisfeitas:

(i) A é um H-módulo twisted, ou seja,

$$
\begin{gathered}
1_{H} \cdot a=a, \text { para todo } a \in A . \\
h \cdot(l \cdot a)=\sum \sigma\left(h_{(1)}, l_{(1)}\right)\left(h_{(2)} l_{(2)} \cdot a\right) \sigma^{-1}\left(h_{(3)}, l_{(3)}\right), \text { para todos } h, l \in H \text { e todo } a \in A .
\end{gathered}
$$

(ii) $\sigma$ for um cociclo, ou seja,

$$
\begin{gathered}
\sigma(h, 1)=\sigma(1, h)=\varepsilon(h) 1_{A}, \text { para todo } h \in H . \\
\sum\left(h_{(1)} \cdot \sigma\left(l_{(1)}, m_{(1)}\right)\right) \sigma\left(h_{(2)}, l_{(2)} m_{(2)}\right)=\sum \sigma\left(h_{(1)}, l_{(1)}\right) \sigma\left(h_{(2)} l_{(2)}, m\right), \text { para todos } h, l, m \in H .
\end{gathered}
$$


Observação. Note que se $\sigma$ for trivial, ou seja, se $\sigma=\mathrm{Id}$, então o produto cruzado nada mais é do que o produto smash álgebra de $A$ e $H$, ou seja, $A \#_{\text {Id }} H=A \# H$. Assim o produto smash é um caso particular do produto cruzado.

\subsubsection{Comódulos e comódulo álgebras}

Com o objetivo de definir apropriadamente a noção de comódulo, vamos expressar a definição de módulo através de diagramas comutativos.

Definição. Seja $A$ uma álgebra, seja $M$ um espaço vetorial e seja $\psi: A \otimes M \longrightarrow M$ uma aplicação linear. Dizemos que $(M, \psi)$ é um $A$-módulo à esquerda se os seguintes diagramas forem comutativos:
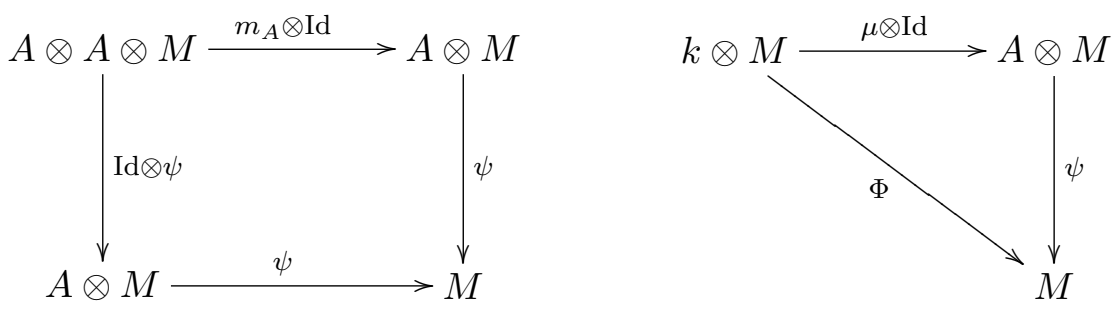

Note que $\Phi$ é o isomorfismo canônico entre os espaços $k \otimes M$ e $M$, ou seja, $\Phi(\lambda \otimes m)=\lambda m$, para todo $\lambda \in k$ e todo $m \in M$. Conforme citado anteriormente, usualmente denotamos $\psi(a \otimes m)=a \cdot m$, para todo $a \in A$ e todo $m \in M$.

Ao dualizar a definição de módulo dada acima, obtemos a seguinte definição de comódulo.

Definição. Seja $C$ uma coálgebra, seja $M$ um espaço vetorial e seja $\rho: M \longrightarrow M \otimes C$ uma aplicação linear. Dizemos que $(M, \rho)$ é um $C$-comódulo à direita se os seguintes diagramas forem comutativos:
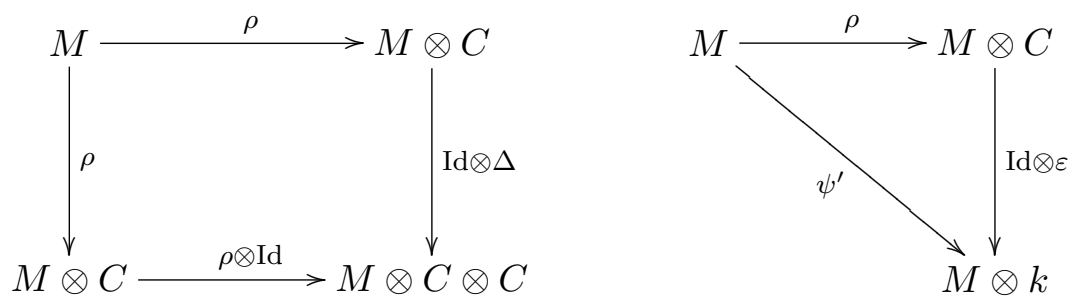

Observação. Note que $\psi^{\prime}$ é o isomorfismo canônico entre os espaços $M$ e $M \otimes k$, ou seja, $\psi^{\prime}(m)=$ $m \otimes 1$, para todo $m \in M$. A estrutura de $C$-comódulo à esquerda é definida analogamente. Comódulos 
também possuem uma notação sigma. Seja $M$ um comódulo à direita e seja $m \in M$. Denotamos $\rho(m)=\sum_{(m)} m_{(0)} \otimes m_{(1)}$.

Exemplo 1.3.8. Seja $A$ uma álgebra, seja $G$ um grupo e seja $H=k G$. Então $A$ é um $H$-comódulo à direita se, e somente se, $A$ for uma álgebra $G$-graduada. Neste caso, temos que $A_{\sigma}=\rho^{-1}(A \otimes \sigma)$, para todo $\sigma \in G$.

Demonstração. Seja $A$ uma álgebra $G$-graduada. Da graduação de $A$, podemos escrever $A=\bigoplus_{g \in G} A_{g}$. Vamos definir

$$
\begin{array}{ccc}
\rho: \quad & A & A \otimes H \\
a=\sum_{g \in G} a_{g} & \longmapsto & \sum_{g \in G} a_{g} \otimes g .
\end{array}
$$

É fácil ver que $\rho$ é uma aplicação linear. Além disso, para todo $a \in A$ temos que

$$
((\rho \otimes \operatorname{Id}) \circ \rho)(a)=\sum_{g \in G} a_{g} \otimes g \otimes g=(\operatorname{Id} \otimes \Delta)\left(\sum_{g \in G} a_{g} \otimes g\right)=((\operatorname{Id} \otimes \Delta) \circ \rho)(a)
$$

e

$$
((\operatorname{Id} \circ \varepsilon) \circ \rho)(a)=(\operatorname{Id} \circ \varepsilon)\left(\sum_{g \in G} a_{g} \otimes g\right)=\sum_{g \in G} a_{g} \otimes 1=\psi^{\prime}(a)
$$

Portanto, $(A, \rho)$ é um $H$-comódulo à direita.

Seja $A$ um $H$-comódulo à direita e seja $a \in A$. Podemos escrever $\rho(a)=\sum_{\sigma \in G} a_{\sigma} \otimes \sigma$. Como $\left(\left(\operatorname{Id}_{A} \otimes \Delta\right) \circ \rho\right)(a)=\left(\left(\rho \otimes \operatorname{Id}_{H}\right) \circ \rho\right)(a)$, temos que

$$
\sum_{\sigma \in G} a_{\sigma} \otimes \sigma \otimes \sigma=\sum_{\sigma \in G}\left[\sum_{\tau \in H}\left(a_{\sigma}\right)_{\tau} \otimes \tau \otimes \sigma\right] .
$$

Da expressão acima, temos que

$$
\left(a_{\sigma}\right)_{\tau}= \begin{cases}a_{\sigma} & , \text { se } \sigma=\tau \\ 0 & , \text { se } \sigma \neq \tau\end{cases}
$$

Logo, $\rho\left(a_{\sigma}\right)=a_{\sigma} \otimes \sigma$, para todo $\sigma \in G$. Vamos definir $A_{\sigma}=\left\{a_{\sigma}: a \in A\right\}$, onde $\sigma \in G$. É fácil ver que $A_{\sigma}$ é um subespaço de $A$. 
Pelo segundo diagrama da definição de comódulo, temos que $a=\sum_{\sigma \in G} a_{\sigma}$, para todo $a \in A$. Assim, $A=\sum_{\sigma \in G} A_{\sigma}$. Falta mostrar que a soma é direta.

Observe que $a \in A_{\sigma}$ se, e somente se, $\rho(a)=a \otimes \sigma$. De fato, se $a \in A_{\sigma}$, então $a=b_{\sigma}$, para algum $b \in A$. Conforme vimos anteriormente, $\rho\left(b_{\sigma}\right)=b_{\sigma} \otimes \sigma$, ou seja, $\rho(a)=a \otimes \sigma$. A recíproca é imediata.

Logo, a soma dos $A_{\sigma}$ é direta e $A$ uma álgebra $G$-graduada. Além disso, é fácil ver que $A_{\sigma}=$ $\rho^{-1}(A \otimes \sigma)$, para todo $\sigma \in G$.

Sejam $\left(M, \rho_{M}\right)$ e $\left(N, \rho_{N}\right) C$-comódulos à direita. Dizemos que uma aplicação linear $f: M \longrightarrow N$ é um morfismo de comódulos se $\rho_{N} \circ f=\left(f \otimes \operatorname{Id}_{C}\right) \circ \rho_{M}$.

A partir de agora, sempre que tivermos uma estrutura de comódulo à direita, $\rho$ será a aplicação linear da estrutura, a menos de menção explícita ao contrário.

Da comutatividade do primeiro diagrama da definição dos $C$-comódulos à direita, temos o seguinte lema.

Lema 1.3.9. Seja $C$ uma coálgebra e seja $M$ um $C$-comódulo à direita. Seja $m \in M$ tal que $\rho_{M}(m)=\sum_{i=1}^{n} m_{i} \otimes c_{i}$, onde $\left\{c_{i}\right\}_{1 \leq i \leq n}$ é linearmente independente. Então, $\operatorname{span}_{k}\left\{m_{i}\right\}_{1 \leq i \leq n}$ é um subcomódulo de $M$ de dimensão finita.

A seguir dois teoremas que trazem uma característica de finitude em todos os comódulos e coálgebras. O primeiro foi extraído de [DNR00, Theorem 2.1.7].

Teorema 1.3.10 (Teorema Fundamental dos Comódulos). Seja $C$ uma coálgebra e seja $M$ um $C$-comódulo à direita. Então todo elemento de $M$ pertence a um subcomódulo de $M$ de dimensão finita.

O segundo foi extraído de [DNR00, Theorem 1.4.7]. Note que ele será usado na demonstração do Lema 2.2.2.

Teorema 1.3.11 (Teorema Fundamental das Coálgebras). Todo elemento de uma coálgebra pertence a uma subcoálgebra de dimensão finita.

A partir de agora considere $H$ uma álgebra de Hopf e $A$ uma álgebra. 
Definição. Dizemos que $A$ é uma $H$-comódulo-álgebra à direita se as seguintes condições forem satisfeitas:

1. A é um $H$-comódulo à direita via aplicação linear $\rho$.

2. $\rho(a b)=\rho(a) \rho(b)$, para todos $a, b \in A$. Na notação sigma,

$$
\sum_{(a b)}(a b)_{(0)} \otimes(a b)_{(1)}=\sum_{(a),(b)} a_{(0)} b_{(0)} \otimes a_{(1)} b_{(1)}
$$

3. $\rho\left(1_{A}\right)=1_{A} \otimes 1_{H}$.

Denominamos $A^{\mathrm{co} H}=\{a \in A: \rho(a)=a \otimes 1\}$ a subálgebra de coinvariantes de $A$. Note que $A^{\mathrm{co} H} \subseteq A$ é uma subálgebra de $A$.

Exemplo 1.3.12. Sejam $G$ um grupo, $A$ uma álgebra $G$-graduada e $H=k G$. Então $A$ é uma $H$-comódulo-álgebra à direita e $A^{\mathrm{co} H}=A_{1_{G}}$.

Demonstração. Do Exemplo 1.3.8, temos que $A$ é um $H$-comódulo à direita. Sejam $a=\sum_{g \in G} a_{g}, b=$ $\sum_{h \in G} b_{h} \in A$. Temos

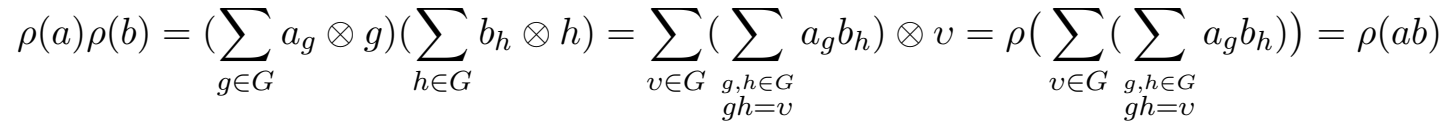

$$
\begin{aligned}
& \rho\left(1_{A}\right)=1_{A} \otimes 1_{G} \text {, pois } 1_{A} \in A_{1_{G}} .
\end{aligned}
$$

Portanto, $A$ é uma $H$-comódulo-álgebra à direita. Observe que $A^{\mathrm{co} H}=A_{1_{G}}$.

As considerações e resultados a seguir serão necessárias para a demonstração da sobrejetividade da correspondência, mais especificamente serão utilizadas na demonstração do Corolário 5.3.4.

Sejam $A, B$ álgebras onde $B \subset A$. Dizemos que $B \subset A$ é uma $H$-extensão à direita se $A$ for uma $H$-comódulo-álgebra à direita e se $A^{\mathrm{co} H}=B$. Dizemos que a extensão tem a propriedade da base 
normal à direita se $A \cong B \otimes H$ como $B$-módulo à esquerda e como $H$-comódulo à direita. Observe que $B \otimes H$ tem estrutura de $H$-comódulo à direita via $\rho=\operatorname{Id}_{B} \otimes \Delta$.

Dizemos ainda que a extensão $A^{\mathrm{co} H} \subseteq A$ é $H$-Galois à direita se a seguinte função

$$
\begin{aligned}
\beta: A \otimes_{A^{\mathrm{co} H}} A & \longrightarrow A \otimes_{k} H \\
a \otimes b & \longmapsto(a \otimes 1) \rho(b)
\end{aligned}
$$

for bijetora.

A seguir um resultado extraído de [Mon93b, Theorem 8.1.7] que trata das extensões $H$-Galois à direita.

Teorema 1.3.13. Seja $G$ um grupo, $A$ uma álgebra $G$-graduada e $H=k G$. Então $A_{1_{G}}=A^{\operatorname{co} H} \subseteq A$ é $H$-Galois à direita se, e somente se, A for fortemente graduada por $G$.

Segue um outro resultado de [Mon93b, Corollary 8.2.5] que trata das extensões $H$-Galois à direita com a propriedade da base normal.

Corolário 1.3.14. Seja $B \subset A$ uma $H$-extensão à direita. Então $B \subset A$ é $H$-Galois à direita com a propriedade da base normal à direita se, e somente se, $A \cong B \#_{\sigma} H$ como álgebras, onde $B \#_{\sigma} H$ é um produto cruzado.

\subsubsection{Coálgebras pontuais e a filtração co-radical}

A partir de agora vamos considerar $C$ uma coálgebra não nula. Dizemos que $C$ é simples se $C$ não possuir subcoálgebras próprias não-nulas, ou seja, se não existir subcoálgebra não-nula $D \subset C$ tal que $D \neq C$. A soma de todas subcoálgebras simples de $C$ é denotada por $C_{0}$ e denominada o co-radical de $C$.

Definição. Dizemos que $C$ é pontual se todas as subcoálgebras simples de $C$ forem unidimensionais.

Observação. Obviamente quando dizemos que uma álgebra de Hopf é pontual estamos nos referindo à sua estrutura de coálgebra.

Segue um resultado extraído de [Swe69, Lemma 8.0.1]. 
Proposição 1.3.15. O conjunto de subcoálgebras unidimensionais de $C$ está em correspondência biunívoca com o conjunto $G(C)$. Além disso, $C$ é pontual se, e somente se, $C_{0}=k G(C)$.

Com o objetivo de definir uma filtração para $C$, seja

$$
C_{n}=\Delta^{-1}\left(C \otimes C_{n-1}+C_{0} \otimes C\right), \text { para } n \geq 1
$$

De [Mon93b, Theorem 5.2.2] temos o seguinte resultado.

Teorema 1.3.16. Para cada $n \geq 0, C_{n}$ é uma subcoálgebra de $C$ e a família $\left\{C_{n}\right\}_{n \geq 0}$ satisfaz:

(i) $C_{n} \subseteq C_{n+1}$ e $C=\bigcup_{n \geq 0} C_{n}$.

(ii) $\Delta\left(C_{n}\right) \subseteq \sum_{i=0}^{n} C_{i} \otimes C_{n-i}$.

Denominamos $\left\{C_{n}\right\}_{n \geq 0}$ a filtração co-radical de $C$. Seja $c \in C$. Dizemos que $c$ tem grau $m$, se $m$ for o menor inteiro tal que $c \in C_{m}$.

Segue mais um importante resultado extraído de [Mon93b, Corollary 5.3.5].

Teorema 1.3.17. Seja $D$ uma coálgebra, e seja $f: C \longrightarrow D$ um morfismo sobrejetor de coálgebras. Então, $D_{0} \subseteq f\left(C_{0}\right)$. Em particular, se $C$ é pontual, então $D_{0}=f\left(C_{0}\right)$ e D também é pontual.

Seguem alguns resultados sobre as álgebras de Hopf pontuais. O primeiro foi extraído de [Mon93b, Corollary 5.2.11].

Teorema 1.3.18. Toda álgebra de Hopf cujo co-radical é cocomutativo possui antípoda bijetora. Em particular, toda álgebra de Hopf pontual tem antípoda bijetora.

Proposição 1.3.19. Seja $H$ uma álgebra de Hopf pontual e seja $\left\{H_{n}\right\}_{n \geq 0}$ a filtração co-radical de H. Então $H_{0} H_{n} \subseteq H_{n}$, para todo $n \geq 0$.

Demonstração. É claro que $H_{0} H_{0} \subseteq H_{0}$. Para o caso em que $n \geq 1$, o resultado é consequência direta da definição $H_{n}=\Delta^{-1}\left(H \otimes H_{n-1}+H_{0} \otimes H\right)$. Vamos por indução em $n$.

Para $n=1$. Seja $x \in H_{1}$ e seja $g \in G(H)$. Temos que

$$
\Delta(g x)=\Delta(g) \Delta(x) \in\left(H_{0} \otimes H_{0}\right)\left(H \otimes H_{0}+H_{0} \otimes H\right) \subseteq H \otimes H_{0}+H_{0} \otimes H .
$$


Da inclusão acima, concluímos que $g x \in H_{1}$. Como $H_{0}=k G(H)$, temos que $H_{0} H_{1} \subseteq H_{1}$.

Agora, vamos supor que o resultado é válido para valores menores do que $n$. Seja $x \in H_{n}$ e seja $g \in G(H)$. Temos que

$$
\begin{aligned}
\Delta(g x) & =\Delta(g) \Delta(x) \in\left(H_{0} \otimes H_{0}\right)\left(H \otimes H_{n-1}+H_{0} \otimes H\right) \\
& \subseteq H \otimes H_{0} H_{n-1}+H_{0} \otimes H \\
& \subseteq H \otimes H_{n-1}+H_{0} \otimes H, \text { pela hipótese indutiva. }
\end{aligned}
$$

Da inclusão acima, concluímos que $g x \in H_{n}$. Portanto, $H_{0} H_{n} \subseteq H_{n}$, para todo $n \geq 0$.

\subsubsection{Integrais}

Antes de falar propriamente de integrais, vamos definir uma estrutura que será importante posteriormente para obtermos um determinado tipo de elemento integral. A próxima definição foi extraída de [DNR00, Remark 3.3.12].

Definição. Dizemos que uma álgebra $A$ de dimensão finita é uma álgebra de Frobenius se $A \cong A^{*}$ como $A$-módulos à esquerda ou, de forma equivalente, como $A$-módulos à direita. Dizemos que uma álgebra $A$ é uma álgebra quasi-Frobenius se $A$ for um $A$-módulo à esquerda injetivo e artiniano. É sabido que uma álgebra de Frobenius é necessariamente quasi-Frobenius.

A seguinte definição foi extraída de [Mas94].

Definição. Dizemos que uma álgebra de Hopf $H$ possui a propriedade (Fr) se todo coideal subálgebra à esquerda e todo coideal subálgebra à direita forem álgebras de Frobenius.

Segue um resultado extraído de [Mas94, Proposition 2.3] que trata da existência da propriedade $(\mathrm{Fr})$.

Proposição 1.3.20. Uma álgebra de Hopf possui a propriedade (Fr) se o co-radical de $H$ for cocomutativo. Em particular, toda álgebra de Hopf pontual possui a propriedade (Fr).

A próxima equivalência foi extraída de [Mas92, Theorem 2.1]. Convém ressaltar que o último item terá um papel fundamental na demonstração da Proposição 5.3.6. 
Teorema 1.3.21. Seja $H$ uma álgebra de Hopf de dimensão finita e seja $B$ um coideal subálgebra à direita de H. Então as seguintes condições são equivalentes:

1. B é uma álgebra de Frobenius;

2. B é uma álgebra quasi-Frobenius;

3. Todo $M$ em ${ }_{B} \mathcal{M}^{H}$ ou em $\mathcal{M}_{B}^{H}$ é um $B$ módulo livre, onde ${ }_{B} \mathcal{M}^{H}$ ( $\left.\mathcal{M}_{B}^{H}\right)$ denota a categoria dos $H$-comódulos à direita que são também B-módulos à esquerda (direita).

Finalmente, vamos aos elementos integrais. Agora vamos denotar por $H$ uma álgebra de Hopf de dimensão finita.

Definição. [Mon93b, Definition 2.1.1] Um integral à esquerda em $H$ é um elemento $t \in H$ tal que $h t=\varepsilon(h) t$, para todo $h \in H$. Um integral à direita em $H$ é um elemento $t^{\prime} \in H$ tal que $t^{\prime} h=\varepsilon(h) t^{\prime}$, para todo $h \in H$.

Vamos denotar por $\int_{H}^{l}$ o espaço de integrais à esquerda, e por $\int_{H}^{r}$ o espaço de integrais à direita. É fácil ver que $\int_{H}^{l} \mathrm{e} \int_{H}^{r}$ são ideais de $H$. Segue um resultado extraído de [Mon93b, Theorem 2.1.3].

Teorema 1.3.22. Seja $H$ uma álgebra de Hopf de dimensão finita. Então:

1. Ambos $\int_{H}^{l} e \int_{H}^{r}$ são unidimensionais.

2. A antipoda $S$ de $H$ é bijetora e $S\left(\int_{H}^{l}\right)=\int_{H}^{r}$.

3. H é uma álgebra de Frobenius.

Seja $t$ um elemento qualquer não nulo de $\int_{H}^{l}$ e seja $h \in H$. Como $\int_{H}^{l}$ é unidimensional, podemos escrever $t h=\lambda_{h} t$, onde $\lambda_{h} \in k$. Considere a seguinte função:

$$
\begin{aligned}
\alpha: H & \longrightarrow k \\
h & \longmapsto \lambda_{h}
\end{aligned}
$$


É fácil verificar que $\alpha$ é um morfismo de álgebras. Por [DNR00, Proposition 4.2.11], temos que $H^{*}$ é uma álgebra de Hopf com antípoda $S^{*}$, e por [DNR00, Proposition 1.4.15], temos que $\alpha \in G\left(H^{*}\right)$. Logo existe $\bar{\alpha}=S^{*}(\alpha)$ a inversa de $\alpha$ no produto de convolução, que é a multiplicação em $H^{*}$.

Seja $t^{\prime}$ um elemento qualquer não nulo de $\int_{H}^{r}$. Como $S\left(t^{\prime}\right) \in \int_{H}^{l}$, temos que

$$
S\left(h t^{\prime}\right)=S\left(t^{\prime}\right) S(h)=\alpha(S(h)) S\left(t^{\prime}\right)=S\left(\alpha(S(h)) t^{\prime}\right) .
$$

Como $S$ é bijetora, $h t^{\prime}=\alpha(S(h)) t^{\prime}$. Mas $\langle\alpha, S(h)\rangle=\left\langle S^{*}(\alpha), h\right\rangle=\langle\bar{\alpha}, h\rangle$. Assim, $h t^{\prime}=\bar{\alpha}(h) t^{\prime}$, para todo $h \in H$.

Definição. O elemento $\alpha \in G\left(H^{*}\right)$ construído acima é chamado o elemento group-like distinguido de $H^{*}$.

Agora vamos tirar a restrição da dimensão de $H$ ser finita.

Definição. Seja $B$ um coideal subálgebra à direita de $H$. Um integral à esquerda em $B$ é um elemento $x \in B$ tal que $b x=\varepsilon(b) x$, para todo $b \in B$. Um integral à direita em $B$ é um elemento $x^{\prime} \in B$ tal que $x^{\prime} b=\varepsilon(b) x^{\prime}$, para todo $b \in B$.

Seguem dois resultados, ambos extraídos de [Kop93], que tratam da existência dos elementos integrais de um coideal subálgebra à direita de $H$. Segue o primeiro, baseado em [Kop93, Theorem $2.2]$.

Teorema 1.3.23. Seja $H$ uma álgebra de Hopf com antípoda bijetora e seja $B$ um coideal subálgebra à direita de $H$ de dimensão finita. As seguintes condições são equivalentes:

(i) B é uma álgebra quasi-Frobenius.

(ii) Existe um integral não nulo à esquerda e um integral nãa nulo à direita em $B$, e pelo menos um deles gera $B$ em $\mathcal{M}^{H}$, onde $\mathcal{M}^{H}$ denota a categoria dos $H$-comódulos à direita, ou seja, $B$ é o menor subcomódulo que contém o elemento integral em questão.

Observação. Observe que $B$ tem uma estrutura natural de $H$-comódulo à direita fazendo $\rho=\Delta$.

Segue o segundo, baseado em [Kop93, Corollary 2.4]. 
Corolário 1.3.24. Seja $H$ uma álgebra de Hopf de dimensão finita e seja $B$ um coideal subálgebra à direita de H. Se ambos os integrais como descrito no Teorema 1.3 .23 (ii) existirem, então eles são os únicos integrais à direita e à esquerda em $B$, a menos de multiplicação por escalar do corpo base $k$.

\subsection{A álgebra de quocientes de Martindale}

O objetivo desta seção é enunciar algumas propriedades do centróide estendido e das álgebras de quocientes de Martindale à esquerda e simétrica. As definições e resultados foram baseados em [Pas87].

A seguir um teorema extraído de [Pas87, Proposition 1.2] que caracteriza a álgebra de quocientes de Martindale à esquerda.

Teorema 1.4.1. Seja $R$ uma álgebra prima. Uma álgebra $Q_{l}$ que satisfaz as condições abaixo está univocamente determinada, a menos de um isomorfismo que se restringe à identidade em $R$. Seguem as condições:

(i) $R$ é uma subálgebra de $Q_{l}$.

(ii) Para todo $q \in Q_{l}$, existe um ideal $A \in \mathcal{F}(R)$ tal que $A q \subseteq R$.

(iii) Para todo $q \in Q_{l}$ e todo ideal $A \in \mathcal{F}(R)$, se $A q=0$, então $q=0$.

(iv) Seja $A \in \mathcal{F}(R)$ e $f: A \rightarrow R$, um homomorfismo de $R$-módulos à esquerda. Então existe $q \in Q_{l}$ tal que $f(a)=a q$, para todo $a \in A$.

Observação. Adicionalmente, temos uma outra condição, consequência direta do item (iii), e por isso denominaremos (iii)'. Segue:

(iii)' Para todo $q \in Q_{l}$ e todo ideal $A \in \mathcal{F}(R)$, se $q A=0$, então $q=0$.

Demonstração. Sejam $q \in Q_{l}$ e $A \in \mathcal{F}(R)$ tais que $q A=0$. Por (ii), existe um ideal $I \in \mathcal{F}(R)$ tal que $I q \subseteq R$. Temos então que $I q R A=0$, já que $R A \subseteq A$ e $q A=0$. Mas $R$ é uma álgebra prima e $I q R$ e $A$ são ideais de $R$ cujo produto é nulo. Como $A \neq 0$, temos que $I q R=0$, ou seja, $I q=0$. Por (iii), concluímos que $q=0$. 
Definição. A álgebra que satisfaz as condições do teorema acima é chamada álgebra de quocientes de Martindale à esquerda de $R$. Vamos denotá-la por $Q_{l}$.

Convém ressaltar que a álgebra de quocientes de Martindale à direita, denotada por $Q_{r}$, é definida de forma análoga. Segue agora um teorema extraído de [Pas87, Proposition 1.4] que caracteriza a álgebra de quocientes de Martindale simétrica.

Teorema 1.4.2. Seja $R$ uma álgebra prima. Uma álgebra $Q$ que satisfaz as condições abaixo está univocamente determinada, a menos de um isomorfismo que se restringe à identidade em $R$. Seguem as condições:

(i) $R$ é uma subálgebra de $Q$.

(ii) Para todo $q \in Q$, existem ideais $A, B \in \mathcal{F}(R)$ tais que $A q, q B \subseteq R$.

(iii) Para todo $q \in Q$ e todo ideal $A \in \mathcal{F}(R)$, se $A q=0$ ou $q A=0$, então $q=0$.

(iv) Sejam $A, B \in \mathcal{F}(R), f: A \rightarrow R$, um homomorfismo de $R$-módulos à esquerda, e $g: B \rightarrow R$, um homomorfismo de $R$-módulos à direita, tais que $f(a) b=a g(b)$, para todos $a \in A$ e $b \in B$. Então existe $q \in Q$ tal que $f(a)=a q$, para todo $a \in A$ e $g(b)=q b$, para todo $b \in B$.

Observação. Verifica-se que $Q=\left\{q \in Q_{l}: q B \subseteq R\right.$, para algum $\left.B \in \mathcal{F}(R)\right\}=\left\{q \in Q_{r}: B q \subseteq R\right.$, para algum $B \in \mathcal{F}(R)\}$.

Definição. A álgebra que satisfaz as condições do teorema acima é chamada álgebra de quocientes de Martindale simétrica de $R$. Vamos denotá-la por $Q$.

O próximo resultado foi extraído de [Pas89, Lemma 10.9] e mostra que ambas as álgebras de quocientes de Martindale à esquerda e simétrica são álgebras primas. Além disso, introduz o centralizador de $R$ em $Q_{l}$, que será denotado por $K$ e que terá papel fundamental no decorrer deste trabalho.

Teorema 1.4.3. Sejam $R$ uma k-álgebra prima e $T=Q_{l}$ ou $T=Q$. Então:

(i) A álgebra $T$ é prima. Mais especificamente, se $q_{1}, q_{2} \in T$ são tais que $q_{1} R q_{2}=0$, então $q_{1}=0$ ou $q_{2}=0$. 
(ii) O centralizador de $R$ em $Q_{l}$ é um corpo e coincide tanto com o centro de $Q_{l}$ quanto com o de $Q$.

Observação. O centralizador de $R$ em $Q_{l}$ chama-se centróide estendido de $R$.

A partir de agora, sempre que $R$ representar uma álgebra prima, $Q_{l}$ será a álgebra de quocientes de Martindale à esquerda de $R, Q_{r}$ será a álgebra de quocientes de Martindale à direita de $R, Q$ será a álgebra de quocientes de Martindale simétrica de $R$ e $K$ será o centróide estendido de $R$. É importante citar que $Q_{l}, Q_{r}$ e $Q$ sempre existem.

\subsection{Ações de álgebra de Hopf em álgebras primas}

O objetivo desta seção é enunciar uma série de resultados a respeito de ações de álgebra de Hopf em álgebras primas.

Definição. Seja $R$ uma álgebra prima e seja $H$ uma álgebra de Hopf que age em $R$. Dizemos que a ação de $H$ em $R$ é contínua se, para todo $h \in H$ e todo ideal $I \in \mathcal{F}(R)$, existir um ideal $J \in \mathcal{F}(R)$ tal que $h \cdot J \subseteq I$.

Conforme enunciado no teorema abaixo, extraído de [Mon93a, Proposition 2.3], ações de álgebras de Hopf pontuais são sempre contínuas.

Teorema 1.5.1. Seja $R$ uma álgebra prima e seja $H$ uma álgebra de Hopf pontual que age em $R$. Então a ação de $H$ em $R$ é contínua.

A seguir um teorema extraído de [Coh86, Proposition 5] que mostra que, em certas condições, a ação de $H$ em $R$ se estende unicamente a uma ação de $H$ em $Q_{l}$.

Teorema 1.5.2. Seja $R$ uma álgebra prima e seja $H$ uma álgebra de Hopf que age em $R$. Suponha que a antípoda $S$ de $H$ seja inversível e que a ação de $H$ em $R$ seja contínua. Então a ação se estende unicamente a uma ação de $H$ em $Q_{l}$.

Nas condições do teorema anterior, mostra-se que a extensão da ação de $H$ em $R$ a uma ação de $H$ em $Q_{l}$ se restringe a uma ação de $H$ em $Q$, conforme enunciado no teorema abaixo, extraído de [Coh86, Proposition 6]. 
Teorema 1.5.3. Seja $R$ uma álgebra prima e seja $H$ uma álgebra de Hopf que age em $R$. Suponha que a antípoda $S$ de $H$ seja inversível e que a ação de $H$ em $R$ seja contínua. Então a extensão da ação de $H$ em $R$ a uma ação de $H$ em $Q_{l}$ se restringe a uma ação de $H$ em $Q$.

Convém lembrar que, pelo Teorema 1.3.18, toda álgebra de Hopf pontual $H$ tem antípoda bijetora, logo inversível. Assim, de acordo com os teoremas enunciados acima, quando se tem uma ação de uma álgebra de Hopf pontual $H$ em uma álgebra prima $R$, podemos estender unicamente essa ação a uma ação de $H$ em $Q_{l}$ que se restringe a uma ação de $H$ em $Q$. 


\section{Capítulo 2}

\section{Mais sobre coálgebras}

\subsection{Introdução}

Apesar de abordarmos as coálgebras no Capítulo 1, precisaremos de vários resultados adicionais sobre essa estrutura para cumprir o objetivo principal deste trabalho. Este capítulo traz estes resultados adicionais.

A Seção 2.2 traz uma série de propriedades de alguns coideais especiais, que serão usados no decorrer deste trabalho, e a Seção 2.3 faz um estudo mais aprofundado sobre a estrutura das coálgebras pontuais.

\subsection{Alguns coideais especiais}

Esta seção tem por objetivo trazer uma série de resultados a respeito de alguns coideais especiais. Esses coideais terão um papel importante nos capítulos subsequentes. 


\subsubsection{Os coideais da forma $L(c)$ e $R(c)$}

Seja $C$ uma coálgebra e seja $c \in C$. Podemos escrever $\Delta(c)=\sum_{i} c_{i} \otimes d_{i}$, onde $c_{i}, d_{i} \in C$, para todo $i$, de modo que $\left\{d_{i}\right\}_{i}$ seja linearmente independente. Dessa forma, definimos

$$
L(c)=\operatorname{span}_{k}\left\{c_{i}\right\}_{i}
$$

De forma análoga se, ao invés de $\left\{d_{i}\right\}_{i}$, o conjunto $\left\{c_{i}\right\}_{i}$ for linearmente independente, definimos

$$
R(c)=\operatorname{span}_{k}\left\{d_{i}\right\}_{i}
$$

Além disso, se $I$ é um subconjunto qualquer de $C$, denotamos

$$
L(I)=\sum_{c \in I} L(c) \quad \text { e } \quad R(I)=\sum_{c \in I} R(c) .
$$

A proposição seguinte mostra que $L(c)$ e $R(c)$ estão bem definidos. Consequentemente, $L(I)$ e $R(I)$ também estão bem definidos.

Proposição 2.2.1. Os subespaços $L(c)$ e $R(c)$ estão bem definidos.

Demonstração. Inicialmente, vamos mostrar que $L(c)$ está bem definido. Seja $\Delta(c)=\sum_{i} c_{i} \otimes d_{i}=$ $\sum_{j} e_{j} \otimes f_{j}$, onde $c_{i}, d_{i}, e_{j}, f_{j} \in C$, para todo $i$ e todo $j$, de modo que $\left\{d_{i}\right\}_{i}$ e $\left\{f_{j}\right\}_{j}$ sejam linearmente independentes.

Vamos considerar $\left\{f_{j}\right\}_{j} \cup\left\{f_{z}^{\prime}\right\}_{z}$ base de $C$. Assim, podemos escrever $d_{i}=\sum_{j} \lambda_{i j} f_{j}+\sum_{z} \lambda_{i z}^{\prime} f_{z}^{\prime}$, onde $\lambda_{i j}, \lambda_{i z}^{\prime} \in k$, para todo $j$, todo $z$ e todo $i$. Então: 


$$
\begin{aligned}
\sum_{i} c_{i} \otimes d_{i} & =\sum_{i} c_{i} \otimes\left(\sum_{j} \lambda_{i j} f_{j}+\sum_{z} \lambda_{i z}^{\prime} f_{z}^{\prime}\right)=\sum_{i}\left[\sum_{j} \lambda_{i j} c_{i} \otimes f_{j}+\sum_{z} \lambda_{i z}^{\prime} c_{i} \otimes f_{z}^{\prime}\right] \\
& =\sum_{i}\left[\sum_{j} \lambda_{i j} c_{i} \otimes f_{j}\right]+\sum_{i}\left[\sum_{z} \lambda_{i z}^{\prime} c_{i} \otimes f_{z}^{\prime}\right] \\
& =\sum_{j}\left(\sum_{i} \lambda_{i j} c_{i}\right) \otimes f_{j}+\sum_{z}\left(\sum_{i} \lambda_{i z}^{\prime} c_{i}\right) \otimes f_{z}^{\prime} .
\end{aligned}
$$

Por outro lado, $\sum_{i} c_{i} \otimes d_{i}=\sum_{j} e_{j} \otimes f_{j}$. Assim, $\sum_{i} \lambda_{i z}^{\prime} c_{i}=0$, para todo $z$, e $\sum_{i} \lambda_{i j} c_{i}=e_{j}$, para todo $j$. Logo, $\operatorname{span}_{k}\left\{e_{j}\right\}_{j} \subseteq \operatorname{span}_{k}\left\{c_{i}\right\}_{i}$. De forma análoga, mostra-se que $\operatorname{span}_{k}\left\{c_{i}\right\}_{i} \subseteq \operatorname{span}_{k}\left\{e_{j}\right\}_{j}$.

Portanto, $L(c)$ está bem definido. De forma análoga, mostra-se que $R(c)$ também está bem definido.

Como podemos escrever $c=\sum_{i} \varepsilon\left(d_{i}\right) c_{i}=\sum_{i} \varepsilon\left(c_{i}\right) d_{i}$, temos que

$$
c \in L(c) \quad \text { e } \quad c \in R(c)
$$

Portanto, temos também

$$
I \subseteq L(I) \quad \text { e } \quad I \subseteq R(I)
$$

Note que, para cada resultado válido para $L(c)$, temos um resultado simétrico válido para $R(c)$. Isto porque $R(c)=L^{\mathrm{cop}}(c)$, onde $L^{\mathrm{cop}}(c)$ denota o espaço $L(c)$ quando olhamos para $c$ na coálgebra co-oposta $C^{\mathrm{cop}}$.

Lema 2.2.2. Seja $c \in C$. Então:

(i) $L L(c)=L(c)$.

(ii) $L(c)$ é um coideal à direita de $C$.

(iii) Se I é um coideal à direita de $C$, então $L(I)=I$. 
(iv) $R R(c)=R(c), R(c)$ é um coideal à esquerda de $C$, e se I é um coideal à esquerda de $C$, então $R(I)=I$.

(v) $D=R L(c)=L R(c)$ e $D$ é uma subcoálgebra de $C$.

(vi) Seja $H$ uma álgebra de Hopf, seja $h \in H$ e seja $\sigma \in G(H)$. Então $\sigma L(h)=L(\sigma h), L(h) \sigma=$ $L(h \sigma), \sigma R(h)=R(\sigma h)$ e $R(h) \sigma=R(h \sigma)$.

Demonstração. Pelo Teorema 1.3.11 (Teorema Fundamental das Coálgebras), todo elemento de uma coálgebra pertence a uma subcoálgebra de dimensão finita. Seja $\left\{d_{i}\right\}_{1 \leq i \leq n}$ uma base de uma subcoálgebra $U \subseteq C$ tal que $c \in U$, e $\Delta\left(d_{i}\right)=\sum_{j=1}^{n} d_{j i} \otimes d_{j}$, onde $d_{j i} \in U$, para todos $1 \leq i, j \leq n$.

Podemos escrever $\Delta(c)=\sum_{i=1}^{n} c_{i} \otimes d_{i}$. Então,

$$
((\Delta \otimes \mathrm{Id}) \circ \Delta)(c)=(\Delta \otimes \mathrm{Id})\left(\sum_{i=1}^{n} c_{i} \otimes d_{i}\right)=\sum_{i=1}^{n} \Delta\left(c_{i}\right) \otimes d_{i} .
$$

Temos ainda,

$$
((\operatorname{Id} \otimes \Delta) \circ \Delta)(c)=(\operatorname{Id} \otimes \Delta)\left(\sum_{i=1}^{n} c_{i} \otimes d_{i}\right)=\sum_{i, j=1}^{n} c_{i} \otimes d_{j i} \otimes d_{j}
$$

Pela coassociatividade da comultiplicação, podemos igualar as expressões acima. Daí,

$$
\Delta\left(c_{i}\right)=\sum_{z=1}^{n} c_{z} \otimes d_{i z}, \text { para todo } 1 \leq i \leq n
$$

Como $d_{i z}$ é uma combinação linear dos $\left\{d_{j}\right\}_{1 \leq j \leq n}$, para todos $1 \leq i, z \leq n$, e como $L(c)=$ $\operatorname{span}_{k}\left\{c_{i}\right\}_{1 \leq i \leq n}$, é fácil concluir que $L\left(c_{i}\right) \subseteq L(c)$, para todo $1 \leq i \leq n$. Assim, $L L(c) \subseteq L(c)$.

Além disso, por (2.2), temos que $L(c) \subseteq L L(c)$. Logo, mostramos que vale (i).

(ii) Observe que $\Delta(L(c)) \subseteq L L(c) \otimes C$. De (i), sabemos que $L L(c)=L(c)$. Logo, $\Delta(L(c)) \subseteq$ $L(c) \otimes C$, e portanto, $L(c)$ é um coideal à direita de $C$. 
(iii) Por (2.1), é imediato que $I \subseteq \sum_{c \in I} L(c)$. Como $I$ é coideal à direita, $L(c) \subseteq I$, para todo $c \in I$. Logo, $L(I)=I$.

(iv) Basta aplicar os itens (i), (ii) e (iii) à coálgebra $C^{\text {cop }}$, que é a coálgebra co-oposta de $C$.

(v) Podemos escrever

$$
((\Delta \otimes \mathrm{Id}) \circ \Delta)(c)=\sum_{i, j, z=1}^{n} \beta_{i j z}\left(d_{i} \otimes d_{j} \otimes d_{z}\right), \text { onde } \beta_{i j z} \in k, \text { para todos } 1 \leq i, j, z \leq n .
$$

Da expressão acima, observe que $R L(c)=\operatorname{span}_{k}\left\{\sum_{j=1}^{n} \beta_{i j z} d_{j}\right\}_{1 \leq i, z \leq n}$. Pela coassociatividade da comultiplicação, temos que

$$
((\operatorname{Id} \otimes \Delta) \circ \Delta)(c)=((\Delta \otimes \operatorname{Id}) \circ \Delta)(c)=\sum_{i, j, z=1}^{n} \beta_{i j z}\left(d_{i} \otimes d_{j} \otimes d_{z}\right) .
$$

Logo, $D=L R(c)=\operatorname{span}_{k}\left\{\sum_{j=1}^{n} \beta_{i j z} d_{j}\right\}_{1 \leq i, z \leq n}=R L(c)$.

De (ii), temos que $L(c)$ é um coideal à direita de $C$. De (iv), temos que $R(c)$ é coideal à esquerda de $C$. Então:

$$
\begin{aligned}
& \Delta(D)=\Delta(R L(c)) \subseteq C \otimes R L(c)=C \otimes D . \\
& \Delta(D)=\Delta(L R(c)) \subseteq L R(c) \otimes C=D \otimes C .
\end{aligned}
$$

Das inclusões acima, temos que $\Delta(D) \subseteq(C \otimes D) \cap(D \otimes C)=D \otimes D$. Logo, $D=R L(c)=L R(c)$ é uma subcoálgebra de $C$.

(vi) Podemos escrever $\Delta(h)=\sum_{i} h_{i} \otimes d_{i}$, onde $h_{i}, d_{i} \in H$, para todo $i$, e $\left\{d_{i}\right\}_{i}$ é linearmente independente. Dessa forma, temos que $L(h)=\operatorname{span}_{k}\left\{h_{i}\right\}_{i}$.

Do fato de $\sigma$ ser inversível, temos que $\left\{\sigma d_{i}\right\}_{i}$ é linearmente independente. Como

$$
\Delta(\sigma h)=\Delta(\sigma) \Delta(h)=\sum_{i} \sigma h_{i} \otimes \sigma d_{i}
$$

temos que $L(\sigma h)=\operatorname{span}_{k}\left\{\sigma h_{i}\right\}_{i}=\sigma \operatorname{span}_{k}\left\{h_{i}\right\}_{i}=\sigma L(h)$. 
Mostram-se as outras identidades de forma análoga.

\subsubsection{Os coideais da forma $H^{Y}$}

No decorrer desta seção, $A$ será uma álgebra e $H$ será uma álgebra de Hopf agindo em $A$. Da Seção 1.3.2, podemos considerar o produto smash $A \# H$. Lembre que podemos identificar $A$ e $H$ com as subálgebras $A \# 1_{H}$ e $1_{A} \# H$ de $A \# H$, respectivamente.

O próximo resultado foi extraído de [Wes00, Lemma 1.1].

Lema 2.2.3. Seja $Y \subseteq A$. Então:

1. $H^{Y}=\{h \in H: L(h)$ age trivialmente em $Y\}$.

2. $H^{Y}$ é um coideal subálgebra à direita de $H$.

3. Seja $\sigma \in G(H)$. Então $\sigma \in H^{Y}$ se, e somente se, $\sigma \cdot y=y$, para todo $y \in Y$.

Demonstração. Seja $h \in H$. Podemos escrever

$$
\Delta(h)=\sum_{i} h_{i} \otimes d_{i}, \text { onde } h_{i}, d_{i} \in H,
$$

de modo que $\left\{d_{i}\right\}_{i}$ seja linearmente independente.

(1) Se $h \in H^{Y}$, temos que

$$
\sum_{i}\left(h_{i} \cdot y\right) \# d_{i}=h y=y h=\sum_{i} \varepsilon\left(h_{i}\right) y \# d_{i} \text {, para todo } y \in Y \text {. }
$$

Assim, $h_{i} \cdot y=\varepsilon\left(h_{i}\right) y$, para todo $i$ e todo $y \in Y$. Portanto,

$$
H^{Y} \subseteq\{h \in H: L(h) \text { age trivialmente em } Y\} .
$$

Se $L(h)$ age trivialmente em $Y$, temos que

$$
h y=\sum_{i}\left(h_{i} \cdot y\right) \# d_{i}=\sum_{i} \varepsilon\left(h_{i}\right) y \# d_{i}=y h, \text { para todo } y \in Y .
$$


Portanto, $H^{Y}=\{h \in H: L(h)$ age trivialmente em $Y\}$.

(2) É fácil ver que $H^{Y}$ é uma subálgebra de $H$. Falta mostrar que $H^{Y}$ é um coideal à direita de $H$.

Por (1), se $h \in H^{Y}$, temos que $L(h)$ age trivialmente em $Y$. Assim, usando a expressão (2.3), temos que $L\left(h_{i}\right) \subseteq L L(h)=L(h)$, para todo $i$, pelo Lema 2.2.2. Então, $L\left(h_{i}\right)$ age trivialmente em $Y$. Novamente por (1), concluímos que $h_{i} \in H^{Y}$. Logo, $H^{Y}$ é um coideal à direita de $H$.

(3) Observe que $L(\sigma)=k \sigma$. Daí, o resultado é imediato a partir de (1).

Lema 2.2.4. Seja $Y \subseteq A$. Se $H^{Y}$ for um coideal à esquerda de $H$ e se a antípoda de $H$ for inversível, então $H^{Y}$ é uma subálgebra de Hopf de $H$.

Demonstração. Pelo Lema 2.2.3, temos que $H^{Y}$ é um coideal subálgebra à direita de $H$ que age trivialmente em $Y$. Mas, por hipótese, $H^{Y}$ é um coideal à esquerda de $H$. Assim, $H^{Y}$ é uma subálgebra e uma subcoálgebra de $H$. Resta mostrar que $S\left(H^{Y}\right) \subseteq H^{Y}$.

Seja $a \in Y$ e seja $h \in H^{Y}$. Temos a seguinte igualdade:

$$
\begin{aligned}
a h & =a \# h=\sum \varepsilon\left(h_{(1)}\right) a \# h_{(2)} \\
& =\sum\left(h_{(2)} \bar{S}\left(h_{(1)}\right)\right) \cdot a \# h_{(3)}, \text { pois } \sum h_{(2)} \bar{S}\left(h_{(1)}\right)=\varepsilon(h) 1_{H} . \\
& =\sum h_{(2)} \cdot\left(\bar{S}\left(h_{(1)}\right) \cdot a\right) \# h_{(3)} \\
& =\sum\left(1_{A} \# h_{(2)}\right)\left(\bar{S}\left(h_{(1)}\right) \cdot a \# 1_{H}\right) \\
& =h_{(2)}\left(\bar{S}\left(h_{(1)}\right) \cdot a\right)
\end{aligned}
$$

Utilizando a igualdade acima, temos que

$$
\begin{aligned}
a S(h) & =\sum S(h)_{(2)}\left(\bar{S}\left(S(h)_{(1)}\right) \cdot a\right) \\
& =\sum S\left(h_{(1)}\right)\left(\bar{S}\left(S\left(h_{(2)}\right)\right) \cdot a\right), S \text { é antimorfismo de coálgebras. } \\
& =\sum S\left(h_{(1)}\right)\left(h_{(2)} \cdot a\right) \\
& =\sum S\left(h_{(1)}\right)\left(\varepsilon\left(h_{(2)}\right) a\right), H^{Y} \text { age trivialmente em } Y . \\
& =\sum S\left(\varepsilon\left(h_{(2)}\right) h_{(1)}\right) a=S(h) a .
\end{aligned}
$$


Assim, $S(h) \in H^{Y}$. Logo, $S\left(H^{Y}\right) \subseteq H^{Y}$.

\subsection{A estrutura de coálgebras pontuais}

Esta seção tem como base o artigo [Mar85], e seu objetivo é chegar em um resultado sobre a estrutura das coálgebras pontuais, que estende o obtido pelo Teorema de Taft-Wilson, enunciado a seguir, cuja demonstração está em [Mon93b, Theorem 5.4.1].

Teorema 2.3.1 (Taft-Wilson). Seja $C$ uma coálgebra pontual e seja $G=G(C)$. Então:

(i) $C_{1}=k G \oplus\left(\bigoplus_{g, h \in G} P_{g, h}^{\prime}(C)\right)$.

(ii) para cada $n \geq 2$ e todo $c \in C_{n}, c=\sum_{g, h \in G} c_{g, h}$, onde $\Delta\left(c_{g, h}\right)=g \otimes c_{g, h}+c_{g, h} \otimes h+w$, para $\operatorname{algum} w \in C_{n-1} \otimes C_{n-1}$.

Observação. Quando combinamos o Teorema de Taft-Wilson com o Teorema 1.3.16, obtemos um resultado um pouco mais refinado para o item (ii). Nota-se na verdade que $w \in \sum_{i=1}^{n-1} C_{i} \otimes C_{n-i}$.

Durante esta seção, $C$ representará sempre uma coálgebra pontual e $\left\{C_{n}\right\}_{n \geq 0}$ a sua filtração co-radical. Por [Mon93b, Theorem 5.4.2], existe um coideal $I$ de $C$ tal que $C=I \oplus C_{0}$. Convém ressaltar que esse coideal $I$ não é único.

Para cada $n \geq 1$, seja $I_{n}=C_{n} \cap I$. Como $C_{0} \subseteq C_{n}$, temos que $C_{n}=I_{n} \oplus C_{0}$. Sejam agora $X_{0}=C_{0}, X_{1}=I_{1}$ e, para cada $n \geq 2$, seja $X_{n}$ subspaço de $C$, tal que $I_{n}=I_{n-1} \oplus X_{n}$. Definindo $C_{-1}=0$, temos que $C_{n}=I_{n} \oplus C_{0}=I_{n-1} \oplus X_{n} \oplus C_{0}=C_{n-1} \oplus X_{n}$, para todo $n \geq 0$.

Na demonstração do Teorema de Taft-Wilson (Teorema 2.3.1) prova-se que

$$
I_{1}=\bigoplus_{g, h \in G} P_{g, h}^{\prime}(C) .
$$

Observação. Para cada $n \geq 1$, note que $I_{n}=\bigoplus_{i=1}^{n} X_{i}$.

Sejam $\sigma, \tau \in G=G(C)$. Definimos, para cada $n \geq 0$, 


$$
\begin{aligned}
& X_{\sigma, \tau, n}=\left\{x \in X_{n}: \Delta(x) \in\left(\sigma \otimes x+C \otimes C_{n-1}\right) \cap\left(x \otimes \tau+C_{n-1} \otimes C\right)\right\} . \\
& X_{\sigma, \tau}=\bigoplus_{n \geq 0} X_{\sigma, \tau, n} .
\end{aligned}
$$

Note que $X_{\sigma, \tau, 0}=\left\{x \in X_{0}: \Delta(x)=\sigma \otimes x=x \otimes \tau\right\}$. Então,

$$
X_{\sigma, \tau, 0}= \begin{cases}k \sigma & , \text { se } \sigma=\tau \\ 0 & , \text { se } \sigma \neq \tau\end{cases}
$$

No caso de $n \geq 1$, é fácil ver que

$$
X_{\sigma, \tau, n}=\left\{x \in X_{n}: \Delta(x) \in \sigma \otimes x+x \otimes \tau+C_{n-1} \otimes C_{n-1}\right\}
$$

(Para essa verificação, basta considerar uma base de $C$ contendo $x$ que estenda uma base de $C_{n-1}$, para cada $\left.x \in X_{\sigma, \tau, n}\right)$.

Proposição 2.3.2. $\quad$ (i) Para todo $n \geq 0$, temos:

$$
X_{n}=\bigoplus_{\sigma, \tau \in G} X_{\sigma, \tau, n}
$$

(ii) $X_{\sigma, \tau, 1} \subset P_{\sigma, \tau}(C)$, para todos $\sigma, \tau \in G$.

Demonstração. (i) O fato de que, para todo $n \geq 0$,

$$
X_{n}=\sum_{\sigma, \tau \in G} X_{\sigma, \tau, n}
$$

é consequência imediata do Teorema de Taft-Wilson (Teorema 2.3.1). Se $n=0$, a soma é claramente direta. Seja $n \geq 1$ e seja $x \in X_{\sigma, \tau, n} \cap X_{\sigma^{\prime}, \tau^{\prime}, n}$, onde $\sigma, \tau, \sigma^{\prime}, \tau^{\prime} \in G$ e $(\sigma, \tau) \neq\left(\sigma^{\prime}, \tau^{\prime}\right)$. Podemos supor sem perda de generalidade que $\sigma \neq \sigma^{\prime}$, daí $\Delta(x)=\sigma \otimes x+w=\sigma^{\prime} \otimes x+w^{\prime}$, onde $w, w^{\prime} \in C \otimes C_{n-1}$. Como $x \in C_{n}$, então $x=0$. Portanto, a soma é direta.

(ii) Seja $x \in X_{\sigma, \tau, 1} \subseteq X_{1}=I_{1}$. De (2.4), podemos escrever $x=\sum_{g, h \in G} c_{g, h}$, onde $c_{g, h} \in P_{g, h}^{\prime}(C)$. Daí

$$
\Delta(x)=\sum_{g, h \in G}\left(g \otimes c_{g, h}+c_{g, h} \otimes h\right) \in \sigma \otimes x+x \otimes \tau+C_{0} \otimes C_{0}
$$


Pelo Teorema de Taft-Wilson (Teorema 2.3.1), a soma $C_{0} \oplus\left(\underset{g, h \in G}{\bigoplus_{g, h}^{\prime}}(C)\right)$ é direta. Assim, é fácil concluir que $\Delta(x)=\sigma \otimes x+x \otimes \tau$, ou seja, $x \in P_{\sigma, \tau}(C)$.

Segue um resultado importante sobre os espaços $X_{\sigma, \tau, n}$ quando estamos em uma álgebra de Hopf pontual.

Proposição 2.3.3. Seja $H$ uma álgebra de Hopf pontual e seja $x \in X_{\sigma, \tau, n} \subseteq H$, onde $n \geq 0$ e $\sigma, \tau \in G(H)$. Então, para todo $\nu \in G(H), \nu x \in X_{\nu \sigma, \nu \tau, n}$.

Demonstração. É claro que vale para o caso em que $n=0$. Vamos mostrar que vale para $n \geq 1$. Da definição de $X_{\sigma, \tau, n}$, temos que $\Delta(x) \in \sigma \otimes x+x \otimes \tau+H_{n-1} \otimes H_{n-1}$. Daí,

$$
\begin{aligned}
\Delta(\nu x) & =\Delta(\nu) \Delta(x) \in(\nu \otimes \nu)\left(\sigma \otimes x+x \otimes \tau+H_{n-1} \otimes H_{n-1}\right) \\
& =\nu \sigma \otimes \nu x+\nu x \otimes \nu \tau+\nu H_{n-1} \otimes \nu H_{n-1} \\
& \subseteq \nu \sigma \otimes \nu x+\nu x \otimes \nu \tau+H_{n-1} \otimes H_{n-1} \text {, pela Proposição 1.3.19. }
\end{aligned}
$$

Pela definição de $X_{\nu \sigma, \nu \tau, n}$, concluímos que $x \in X_{\nu \sigma, \nu \tau, n}$.

Definimos $L_{0}=\{0\}, L_{i}=\bigoplus_{j=1}^{i} X_{j}$, para $i \geq 1$, e $L_{\sigma, \tau}=\bigoplus_{n \geq 1} X_{\sigma, \tau, n}$. Para $i \geq 0$ e $g, h \in G$, sejam $\pi_{i}$ e $\pi_{g, h}$ as projeções naturais de $C$ em $X_{i}$ e $X_{g, h}$, respectivamente. Denotemos por $\pi_{g, h, i}$ a composta $\pi_{i} \circ \pi_{g, h}$, que é a projeção em $X_{g, h, i}$.

Proposição 2.3.4. Para todo $i \geq 1$ e todo $c \in X_{i}$, temos $\left(\left(\pi_{0} \otimes \pi_{0}\right) \circ \Delta\right)(c)=0$ e $\varepsilon(c)=0$.

Demonstração. Como $c \in X_{i} \subseteq I$, que é um coideal de $C$, temos $\varepsilon(c)=0$. Além disso,

$$
\Delta(c) \subseteq C \otimes I+I \otimes C=C_{0} \otimes I+I \otimes C_{0}+I \otimes I
$$

Assim concluímos que $\left(\left(\pi_{0} \otimes \pi_{0}\right) \circ \Delta\right)(c)=0$.

A seguir o teorema principal desta seção, baseado no artigo [Mar85, Theorem 2.1], que estende a descrição feita no Teorema de Taft-Wilson (Teorema 2.3.1) quando a comultiplicação é aplicada em um elemento da coálgebra $C$. 
Teorema 2.3.5. Para todos $g, h \in G$ e todo $x \in L_{g, h}$, temos que

$$
\Delta(x) \in g \otimes x+x \otimes h+\sum_{v \in G} L_{g, v} \otimes L_{v, h}
$$

Demonstração. Sejam $g, h \in G$. Vamos mostrar por indução em $n$ que o teorema vale para todo $x \in X_{i}$. É fácil ver que vale para $x \in X_{g, h, 1}$, a partir da Proposição 2.3.2. Vamos supor que vale para $x \in X_{g, h, i-1}=X_{i-1} \cap L_{g, h}$.

Seja $c \in X_{g, h, i}$. Pelo Teorema de Taft-Wilson (Teorema 2.3.1), temos:

$$
\begin{aligned}
\Delta(c) & \in g \otimes c+c \otimes h+\sum_{j=1}^{i-1} C_{j} \otimes C_{i-j} \\
& =g \otimes c+c \otimes h+\sum_{j=1}^{i-1}\left(C_{0} \oplus L_{j}\right) \otimes\left(C_{0} \oplus L_{i-j}\right) \\
& =g \otimes c+c \otimes h+\left(\sum_{j=1}^{i-1} L_{j} \otimes L_{i-j}\right)+\left(C_{0} \otimes L_{i-1}\right)+\left(L_{i-1} \otimes C_{0}\right)+\left(C_{0} \otimes C_{0}\right) .
\end{aligned}
$$

Pela Proposição 2.3.4, podemos eliminar o termo $C_{0} \otimes C_{0}$ da expressão acima. Gostaríamos de eliminar também os termos $C_{0} \otimes L_{i-1}$ e $L_{i-1} \otimes C_{0}$. Denotando por $\psi=\sum_{j=1}^{i-1} \pi_{j}$, a projeção de $C$ em $L_{i-1}$, podemos escrever:

$$
\begin{aligned}
& \left(\left(\psi \otimes \pi_{0}\right) \circ \Delta\right)(c)=\sum_{b \in G} l_{b} \otimes b, \text { onde } l_{b} \in L_{i-1}, \text { para todo } b \in G . \\
& \left(\left(\pi_{0} \otimes \psi\right) \circ \Delta\right)(c)=\sum_{b \in G} b \otimes r_{b}, \text { onde } r_{b} \in L_{i-1}, \text { para todo } b \in G .
\end{aligned}
$$

Para provar que os elementos acima são nulos, aplicaremos $\Delta \otimes \operatorname{Id}$ e $\operatorname{Id} \otimes \Delta$ em (2.5). Pela coassociatividade da comultiplicação, os elementos obtidos deverão ser iguais. Em particular, as componentes desses elementos em $L_{i-1} \otimes C_{0} \otimes C_{0}$ e em $C_{0} \otimes C_{0} \otimes L_{i-1}$ deverão coincidir. 
(i) Observe que a componente de $((\operatorname{Id} \otimes \Delta) \circ \Delta)(c)$ em $L_{i-1} \otimes C_{0} \otimes C_{0}$ é

$$
\left((\operatorname{Id} \otimes \Delta) \circ\left(\Psi \otimes \pi_{0}\right) \circ \Delta\right)(c)
$$

e a componente de $((\Delta \otimes \mathrm{Id}) \circ \Delta)(c)$ em $L_{i-1} \otimes C_{0} \otimes C_{0}$ é

$$
\left(\left(\mathrm{Id} \otimes \pi_{0} \otimes \mathrm{Id}\right) \circ(\Delta \otimes \mathrm{Id}) \circ\left(\Psi \otimes \pi_{0}\right) \circ \Delta\right)(c)+\left(\left(\Psi \otimes \pi_{0}\right) \circ \Delta\right)(c) \otimes h .
$$

Daí e da equação (2.6), temos que

$$
\sum_{b \in G} l_{b} \otimes b \otimes b=\sum_{b \in G}\left(\left(\operatorname{Id} \otimes \pi_{0}\right) \circ \Delta\right)\left(l_{b}\right) \otimes b+\sum_{b \in G} l_{b} \otimes b \otimes h .
$$

(ii) Observe que a componente de $((\operatorname{Id} \otimes \Delta) \circ \Delta)(c)$ em $C_{0} \otimes C_{0} \otimes L_{i-1}$ é

$$
\left(\left(\mathrm{Id} \otimes \pi_{0} \otimes \mathrm{Id}\right) \circ(\mathrm{Id} \otimes \Delta) \circ\left(\pi_{0} \otimes \Psi\right) \circ \Delta\right)(c)+g \otimes\left(\left(\pi_{0} \otimes \Psi\right) \circ \Delta\right)(c),
$$

e a componente de $((\Delta \otimes \mathrm{Id}) \circ \Delta)(c)$ em $C_{0} \otimes C_{0} \otimes L_{i-1}$ é

$$
\left((\Delta \otimes \mathrm{Id}) \circ\left(\pi_{0} \otimes \Psi\right) \circ \Delta\right)(c) .
$$

Daí e da equação (2.7), temos que

$$
\sum_{b \in G} b \otimes\left(\left(\pi_{0} \otimes \mathrm{Id}\right) \circ \Delta\right)\left(r_{b}\right)+\sum_{b \in G} g \otimes b \otimes r_{b}=\sum_{b \in G} b \otimes b \otimes r_{b}
$$

De (i) e (ii), temos:

$$
\begin{gathered}
\left(\left(\mathrm{Id} \otimes \pi_{0}\right) \circ \Delta\right)\left(l_{\nu}\right)=l_{\nu} \otimes \nu, \text { para todo } \nu \in G, \operatorname{com} \nu \neq h . \\
\left(\left(\operatorname{Id} \otimes \pi_{0}\right) \circ \Delta\right)\left(l_{h}\right)=-\sum_{\substack{b \in G \\
b \neq h}} l_{b} \otimes b . \\
\left(\left(\pi_{0} \otimes \mathrm{Id}\right) \circ \Delta\right)\left(r_{\nu}\right)=\nu \otimes r_{\nu}, \text { para todo } \nu \in G, \operatorname{com} \nu \neq g . \\
\left(\left(\pi_{0} \otimes \mathrm{Id}\right) \circ \Delta\right)\left(r_{g}\right)=-\sum_{\substack{b \in G \\
b \neq g}} b \otimes r_{b} .
\end{gathered}
$$


Como $((\varepsilon \otimes \mathrm{Id}) \circ \Delta)(c)=\left((\varepsilon \otimes \mathrm{Id}) \circ\left(\pi_{0} \otimes \Psi\right) \circ \Delta\right)(c)+1_{k} \otimes c$ e $((\mathrm{Id} \otimes \varepsilon) \circ \Delta)(c)=((\mathrm{Id} \otimes \varepsilon) \circ(\Psi \otimes$ $\left.\left.\pi_{0}\right) \circ \Delta\right)(c)+c \otimes 1_{k}$, temos

$$
l_{h}=-\sum_{\substack{b \in G \\ b \neq h}} l_{b} \text { e } r_{g}=-\sum_{\substack{b \in G \\ b \neq g}} r_{b} .
$$

Seja $c_{1}=c+l_{h}$. Temos que

$$
\begin{aligned}
\left(\left(\operatorname{Id} \otimes \pi_{0}\right) \circ \Delta\right)\left(c_{1}\right) & =\left(\left(\operatorname{Id} \otimes \pi_{0}\right) \circ \Delta\right)(c)+\left(\left(\operatorname{Id} \otimes \pi_{0}\right) \circ \Delta\right)\left(l_{h}\right) \\
& =c \otimes h+\left(\left(\Psi \otimes \pi_{0}\right) \circ \Delta\right)(c)+\left(\left(\operatorname{Id} \otimes \pi_{0}\right) \circ \Delta\right)\left(l_{h}\right), \text { por }(2.5) . \\
& =c \otimes h+\sum_{b \in G} l_{b} \otimes b+\left(-\sum_{\substack{b \in G \\
b \neq h}} l_{b} \otimes b\right), \text { pelas equações }(2.6) \text { e }(2.9) . \\
& =c \otimes h+l_{h} \otimes h \\
& =c_{1} \otimes h
\end{aligned}
$$

Assim, $c_{1}=c+l_{h} \in \sum_{\sigma \in G} L_{\sigma, h}$, e aí $l_{h} \in \sum_{\sigma \in G} L_{\sigma, h}$, já que $c \in L_{g, h}$. Mas pela hipótese de indução e pela equação (2.8), $l_{\nu} \in \sum_{\sigma \in G} L_{\sigma, \nu}$, para todo $\nu \in G \operatorname{com} \nu \neq h$. Da equação (2.12) e da decomposição $C=C_{0} \oplus\left(\bigoplus_{g, h \in G} L_{g, h}\right)$, concluímos que $l_{b}=0$, para todo $b \in G$.

De forma análoga, $r_{b}=0$, para todo $b \in G$. Assim, eliminamos os termos $C_{0} \otimes L_{i-1}$ e $L_{i-1} \otimes C_{0}$ da expressão (2.5). Falta mostrar que $\Delta(c)-g \otimes c-c \otimes h \in \sum_{\nu \in G} L_{g, \nu} \otimes L_{\nu, h}$. Podemos escrever:

$$
((\Psi \otimes \Psi) \circ \Delta)(c)=\sum_{u, v, x, y \in G} l_{u, v} \otimes l_{x, y}^{\prime} \text {, onde } l_{u, v} \in L_{u, v}, l_{x, y}^{\prime} \in L_{x, y} \text { e } l_{u, v}, l_{x, y}^{\prime} \neq 0
$$

Como fizemos anteriormente, vamos aplicar $\Delta \otimes \operatorname{Id}$ e $\operatorname{Id} \otimes \Delta$ em (2.5). Pela coassociatividade da comultiplicação, os elementos obtidos deverão ser iguais. Em particular, as componentes desses elementos em $C_{0} \otimes L_{i-1} \otimes L_{i-1}, L_{i-1} \otimes L_{i-1} \otimes C_{0}$ e em $L_{i-1} \otimes C_{0} \otimes L_{i-1}$ deverão coincidir.

(iii) Observe que a componente de $((\operatorname{Id} \otimes \Delta) \circ \Delta)(c)$ em $C_{0} \otimes L_{i-1} \otimes L_{i-1}$ é

$$
g \otimes((\Psi \otimes \Psi) \circ \Delta)(c)
$$


e a componente de $((\Delta \otimes \mathrm{Id}) \circ \Delta)(c)$ em $C_{0} \otimes L_{i-1} \otimes L_{i-1}$ é

$$
\sum_{u, v, x, y \in G}\left(\left(\pi_{0} \otimes \Psi\right) \circ \Delta\right)\left(l_{u, v}\right) \otimes l_{x, y}^{\prime}
$$

(iv) Observe que a componente de $((\operatorname{Id} \otimes \Delta) \circ \Delta)(c)$ em $L_{i-1} \otimes L_{i-1} \otimes C_{0}$ é

$$
\sum_{u, v, x, y \in G} l_{u, v} \otimes\left(\left(\Psi \otimes \pi_{0}\right) \circ \Delta\right)\left(l_{x, y}^{\prime}\right)
$$

e a componente de $((\Delta \otimes \mathrm{Id}) \circ \Delta)(c)$ em $L_{i-1} \otimes L_{i-1} \otimes C_{0}$ é

$$
((\Psi \otimes \Psi) \circ \Delta)(c) \otimes h
$$

(v) Observe que a componente de $((\operatorname{Id} \otimes \Delta) \circ \Delta)(c)$ em $L_{i-1} \otimes C_{0} \otimes L_{i-1}$ é

$$
\sum_{u, v, x, y \in G} l_{u, v} \otimes\left(\left(\pi_{0} \otimes \Psi\right) \circ \Delta\right)\left(l_{x, y}^{\prime}\right)
$$

e a componente de $((\Delta \otimes \mathrm{Id}) \circ \Delta)(c)$ em $L_{i-1} \otimes C_{0} \otimes L_{i-1}$ é

$$
\sum_{u, v, x, y \in G}\left(\left(\Psi \otimes \pi_{0}\right) \circ \Delta\right)\left(l_{u, v}\right) \otimes l_{x, y}^{\prime} .
$$

Como $l_{u, v} \in L_{u, v}$ e $l_{x, y}^{\prime} \in L_{x, y}$, sendo ambos não nulos, e utilizando a hipótese indutiva, obtemos:

$$
\sum_{u, v, x, y \in G} l_{u, v} \otimes v \otimes l_{x, y}^{\prime}=\sum_{u, v, x, y \in G} l_{u, v} \otimes x \otimes l_{x, y}^{\prime} .
$$

De (iii), (iv) e (v), concluímos que $u=g, y=h$ e que $v=x$. Logo,

$$
\Delta(c) \in g \otimes c+c \otimes h+\sum_{\nu \in G} L_{g, \nu} \otimes L_{\nu, h}
$$

Isso mostra que o resultado vale para $X_{g, h, i}=X_{i} \cap L_{g, h}$, para todo $g, h \in G$. Assim, completamos a indução e a demostração.

A seguir dois corolários, que apesar de imediatos, serão muito utilizados no decorrer do texto. O 
primeiro é consequência direta do Teorema 2.3.5 e do teorema de Taft-Wilson (Teorema 2.3.1). O segundo é consequência direta do primeiro corolário.

Corolário 2.3.6. Sejam $\sigma, \tau \in G$ e $n \geq 1$. Então, para cada $x \in X_{\sigma, \tau, n}$,

$$
\Delta(x) \in \sigma \otimes x+x \otimes \tau+\sum_{\nu \in G}\left[\sum_{\substack{0<i, j<n \\ i+j \leq n}}\left(X_{\sigma, \nu, i} \otimes X_{\nu, \tau, j}\right)\right] .
$$

Corolário 2.3.7. Sejam $\sigma, \tau \in G$ e $n \geq 1$. Então, para cada $x \in X_{\sigma, \tau, n}$,

$$
\Delta(x) \in \sigma \otimes x+x \otimes \tau+\sum_{\substack{0<i, j<n \\ i+j \leq n}} X_{i} \otimes X_{j} .
$$




\section{Capítulo 3}

\section{As ações X-externas}

\subsection{Introdução}

Este capítulo tem por objetivo definir e mostrar diversas propriedades das ações X-externas. Na Seção 3.2 temos uma série de propriedades do produto smash. Na Seção 3.3 definimos o que seriam as chamadas ações X-externas. Finalmente, na Seção 3.4 mostramos algumas propriedades fundamentais das ações X-externas.

No decorrer deste capítulo, $R$ será uma álgebra prima e $H$ será uma álgebra de Hopf pontual agindo em $R$. Convém lembrar que, pelo Teorema 1.3.18, $H$ possui antípoda bijetora. Da Seção 1.5, temos que a ação de $H$ em $R$ é contínua e, portanto, se estende unicamente a uma ação de $H$ em $Q_{l}$ que se restringe a uma ação de $H$ em $Q$. Assim estão definidos os seguintes produtos smash, $R \# H \subseteq Q \# H \subseteq Q_{l} \# H$.

Antes de prosseguirmos, veremos dois resultados importantes. O primeiro resultado, extraído de [Yan97, Lemma 2.4], justifica a notação para a subálgebra de invariantes de $R$ pela ação de $H$, conceito definido na Seção 1.3.2. Observe que estamos identificando $R$ e $H$ com as subálgebras $R \# 1_{H}$ e $1_{R} \# H$ de $R \# H$, respectivamente.

Lema 3.1.1. $O$ conjunto $R^{H}$ coincide com a subálgebra de invariantes de $R$ sobre a ação de $H$, isto é, $R^{H}=\{r \in R: h \cdot r=\varepsilon(h) r$, para todo $h \in H\}$. 
Demonstração. Seja $r \in R$ e seja $h \in H$. Podemos escrever $\Delta(h)=\sum_{i=1}^{n} h_{i}^{\prime} \otimes h_{i}$, onde $\left\{h_{i}\right\}_{1 \leq i \leq n}$ é linearmente independente. Temos então:

$$
\begin{gathered}
h r=(1 \# h)(r \# 1)=\sum_{i=1}^{n} h_{i}^{\prime} \cdot r \# h_{i} \\
r h=r \# h=\sum_{i=1}^{n} \varepsilon\left(h_{i}^{\prime}\right) r \# h_{i}
\end{gathered}
$$

A partir das expressões acima é fácil ver que se $r$ for um elemento invariante pela ação de $H$, então $h r=r h$, para todo $h \in H$.

Se $r$ é tal que $h r=r h$, para todo $h \in H$, temos que $\sum_{i=1}^{n} h_{i}^{\prime} \cdot r \# h_{i}=\sum_{i=1}^{n} \varepsilon\left(h_{i}^{\prime}\right) r \# h_{i}=r \# h$. Aplicando $\operatorname{Id} \otimes \varepsilon$ na igualdade acima, concluímos que $h \cdot r=\varepsilon(h) r$, ou seja, $r$ é um elemento invariante pela ação de $H$.

Logo, $R^{H}=\{r \in R: h \cdot r=\varepsilon(h) r$, para todo $h \in H\}$.

Observe que, no lema anterior, podemos substituir $H$ por um coideal à direita $I$ de $H$, e substituir $R$ por um subconjunto $Y$ de $R$. Pela demonstração acima, é fácil ver que

$$
Y^{I}=\{r \in Y: I \text { age trivialmente em } r\}
$$

Convém ressaltar a semelhança deste resultado com o resultado do Lema 2.2.3 (1). Além disso, da demonstração do Lema 2.2.3 (1), verifica-se facilmente que

$$
I^{Y}=\{h \in I: L(h) \text { age trivialmente em } Y\}
$$

O segundo resultado justifica o fato de dizermos que uma subálgebra $\Lambda$ de $Q \# H$ é uma $H$ comódulo-álgebra à direita, conforme definido na Seção 1.3.3, se $\left(\operatorname{Id}_{Q} \otimes \Delta\right)(\Lambda) \subseteq \Lambda \otimes H$. Observe que estamos fazendo $\rho=\operatorname{Id}_{Q} \otimes \Delta$. A partir de agora deixaremos isto implícito.

Proposição 3.1.2. Seja $\Lambda$ uma subálgebra de $Q \# H$ tal que $\left(\operatorname{Id}_{Q} \otimes \Delta\right)(\Lambda) \subseteq \Lambda \otimes H$. Então $\Lambda$ é uma $H$-comódulo-álgebra à direita via $\rho=\operatorname{Id}_{Q} \otimes \Delta$. 
Demonstração. Seja $\xi=\sum q_{i} \# h_{i} \in \Lambda$. Temos:

$$
\begin{aligned}
\left(\left(\operatorname{Id}_{\Lambda} \otimes \Delta\right) \circ \rho\right)(\xi) & =\left(\operatorname{Id}_{\Lambda} \otimes \Delta\right)\left(\sum q_{i} \# \Delta\left(h_{i}\right)\right) \\
& =\sum q_{i} \#\left[\left(\left(\operatorname{Id}_{H} \otimes \Delta\right) \circ \Delta\right)\left(h_{i}\right)\right], \text { observe que } \operatorname{Id}_{\Lambda}=\operatorname{Id}_{Q} \otimes \operatorname{Id}_{H} . \\
& =\sum q_{i} \#\left[\left(\left(\Delta \otimes \operatorname{Id}_{H}\right) \circ \Delta\right)\left(h_{i}\right)\right] \\
& =\left(\operatorname{Id}_{Q} \otimes \Delta \otimes \operatorname{Id}_{H}\right)\left(\sum q_{i} \# \Delta\left(h_{i}\right)\right) \\
& =\left(\left(\rho \otimes \operatorname{Id}_{H}\right) \circ \rho\right)(\xi) . \\
\left(\left(\operatorname{Id}_{\Lambda} \otimes \varepsilon\right) \circ \rho\right)(\xi) & =\left(\operatorname{Id}_{\Lambda} \otimes \varepsilon\right)\left(\sum q_{i} \# \Delta\left(h_{i}\right)\right) \\
& =\sum q_{i} \#\left[\left(\left(\operatorname{Id}_{H} \otimes \varepsilon\right) \circ \Delta\right)\left(h_{i}\right)\right], \text { observe que } \operatorname{Id}_{\Lambda}=\operatorname{Id}_{Q} \otimes \operatorname{Id}_{H} . \\
& =\sum q_{i} \# h_{i} \\
& =\xi .
\end{aligned}
$$

Assim, $\Lambda$ é um $H$-comódulo à direita via $\rho$. Falta mostrar as outras duas condições da definição de $H$-comódulo-álgebra à direita feita na Seção 1.3.3.

Seja $\xi^{\prime}=\sum q_{j}^{\prime} \# h_{j}^{\prime} \in \Lambda$. Temos:

$$
\begin{aligned}
\rho\left(\xi \xi^{\prime}\right) & =\left(\operatorname{Id}_{Q} \otimes \Delta\right)\left(\left[\sum q_{i} \# h_{i}\right]\left[\sum q_{j}^{\prime} \# h_{j}^{\prime}\right]\right) \\
& =\left(\operatorname{Id}_{Q} \otimes \Delta\right)\left(\sum q_{i}\left(h_{i_{(1)}} \cdot q_{j}^{\prime}\right) \# h_{i_{(2)}} h_{j}^{\prime}\right) \\
& =\sum q_{i}\left(h_{i_{(1)}} \cdot q_{j}^{\prime}\right) \# \Delta\left(h_{i_{(2)}}\right) \Delta\left(h_{j}^{\prime}\right) \\
& =\sum q_{i}\left(h_{i_{(1)}} \cdot q_{j}^{\prime}\right) \# h_{i_{(2)}} h_{j_{(1)}}^{\prime} \otimes h_{i_{(3)}} h_{j_{(2)}}^{\prime} \\
& =\left[\sum\left(q_{i} \# h_{i_{(1)}}\right) \otimes h_{i_{(2)}}\right]\left[\sum\left(q_{j}^{\prime} \# h_{j_{(1)}}^{\prime}\right) \otimes h_{j_{(2)}}^{\prime}\right] \\
& =\rho(\xi) \rho\left(\xi^{\prime}\right) .
\end{aligned}
$$

Note que $1_{Q} \# 1_{H}=1_{\Lambda} \in \Lambda$, pois $\Lambda$ é subálgebra de $Q \# H$. Daí, $\rho\left(1_{\Lambda}\right)=1_{\Lambda} \otimes 1_{H}$, e $\Lambda$ é de fato uma $H$-comódulo-álgebra à direita. 


\subsection{Propriedades dos produtos smash}

Esta seção é baseada no artigo [Mil95a] e destaca uma série de propriedades das álgebras produtos smash. Esses resultados serão muito importantes para os capítulos subsequentes.

Inicialmente vamos mostrar dois lemas fundamentais, ambos baseado em [Mil95a, Lemma 1.3]. Note que, se $H$ age em uma álgebra $A$ e $V$ é um coideal à esquerda de $H$, então $A \# V$ é um $A$-submódulo à esquerda e à direita de $A \# H$.

Lema 3.2.1. Seja A uma álgebra que sofre a ação de $H$, e seja $V$ um coideal à esquerda de $H$. Então:

(1) Se $\left\{h_{\beta}\right\}$ é uma $k$-base de $V$, então $\left\{1 \# h_{\beta}\right\}$ é uma base para o A-módulo à esquerda $A \# V$.

(2) Se $\left\{h_{\beta}\right\}$ é uma $k$-base de $V$, então $\left\{1 \# h_{\beta}\right\}$ é uma base para o A-módulo à direita $A \# V$.

Observação. Note que, para os itens (1) e (2), não é necessário que $H$ seja pontual. O resultado é válido para uma álgebra de Hopf qualquer. A única restrição está no item (2), onde $H$ deve ter, necessariamente, a antípoda bijetora.

Demonstração. (1) É fácil ver que $\left\{1 \# h_{\beta}\right\}$ gera o $A$-módulo à esquerda $A \# V$. Falta mostrar a independência linear.

Seja $\left\{a_{\alpha}\right\}$ uma $k$-base de $A$ e sejam $a_{\beta} \in A$ tais que $\sum_{\beta} a_{\beta}\left(1 \# h_{\beta}\right)=0$. Para cada $\beta$, podemos escrever $a_{\beta}=\sum_{\alpha} \lambda_{\alpha \beta}\left(a_{\alpha}\right)$, onde $\lambda_{\alpha \beta} \in k$. Então,

$$
\sum_{\alpha, \beta} \lambda_{\alpha \beta}\left(a_{\alpha} \# h_{\beta}\right)=0
$$

Mas $\left\{a_{\alpha} \# h_{\beta}\right\}$ é base do espaço vetorial $A \otimes H$. Logo $\lambda_{\alpha \beta}=0$, para todos $\alpha$ e $\beta$, ou seja, $a_{\beta}=0$, para todos $\beta$. Logo $\left\{1 \# h_{\beta}\right\}$ é uma base para o $A$-módulo à esquerda $A \# V$.

(2) Vamos definir $\varphi: V \otimes A \longrightarrow A \# V$ tal que $\varphi(h \otimes a)=(1 \# h) a$, para todo $h \in V$ e todo $a \in A$. É fácil ver que $\varphi$ é um homomorfismo de $A$-módulos à direita. Na verdade é um isomorfismo.

De fato, seja $\Phi: A \# V \longrightarrow V \otimes A$ tal que $\Phi(a \# h)=\sum_{(h)} h_{(2)} \otimes\left(\bar{S}\left(h_{(1)}\right) \cdot a\right)$, para todo $h \in V$ e todo $a \in A$. Assim temos, para todo $h \in V$ e todo $a \in A$, 


$$
\begin{aligned}
\Phi(\varphi(h \otimes a)) & =\Phi((1 \# h) a)=\Phi\left(\sum_{(h)} h_{(1)} \cdot a \# h_{(2)}\right) \\
& =\sum_{(h)} h_{(3)} \otimes\left(\bar{S}\left(h_{(2)}\right) \cdot\left(h_{(1)} \cdot a\right)\right) \\
& =\sum_{(h)} h_{(3)} \otimes\left(\left(\bar{S}\left(h_{(2)}\right) h_{(1)}\right) \cdot a\right) \\
& =\sum_{(h)} h_{(2)} \otimes\left(\varepsilon\left(h_{(1)}\right) 1_{H} \cdot a\right), \text { pois } \sum_{(h)} \bar{S}\left(h_{(2)}\right) h_{(1)}=\varepsilon(h) . \\
& =h \otimes a .
\end{aligned}
$$

Analogamente, mostra-se que $\varphi \circ \Phi=I d_{A \# V}$.

Como $\left\{h_{\beta} \otimes 1\right\}$ é uma base para o $A$-módulo à direita $V \otimes A$, concluímos, a partir do isomorfismo acima, que $\left\{1 \# h_{\beta}\right\}$ é uma base para o $A$-módulo à direita $A \# V$.

Lema 3.2.2. Seja $V$ um coideal à esquerda de H. Então:

(1) Se $x \in Q_{l} \# V$, então existe um ideal $I \in \mathcal{F}(R)$ tal que $I x \subseteq R \# V$.

(2) Para todo $x \in Q_{l} \# V$ e todo ideal $I \in \mathcal{F}(R)$, se $I x=0$, então $x=0$.

(3) Se $V$ tiver dimensão finita, então para todo $I \in \mathcal{F}(R)$ e todo homomorfismo de $R$-módulos à esquerda $f: I \longrightarrow R \# V$, existe $z \in Q_{l} \# V$ tal que $f(r)=r z$, para todo $r \in I$.

(4) Para todo $x \in Q_{l} \# V$ e todo ideal $I \in \mathcal{F}(R)$, se $x I=0$, então $x=0$.

(5) Se $J \in \mathcal{F}(R)$, então $\left(Q_{l} \# V\right)^{J}=\left(Q_{l} \# V\right)^{R}=\left(Q_{l} \# V\right)^{Q_{l}}$.

Demonstração. (1) Pelo lema anterior, para todo $x \in Q_{l} \# V$, podemos escrever $x=\sum_{\beta} q_{\beta}\left(1 \# h_{\beta}\right)$, onde somente um número finito de $q_{\beta} \in Q_{l}$ é não nulo.

Para cada $\beta$ não nulo, pelo Teorema 1.4.1, existe um $A_{\beta} \in \mathcal{F}(R)$ tal que $A_{\beta} q_{\beta} \subseteq R$. Definimos $I=\bigcap_{\beta} A_{\beta}$, que é um ideal não nulo de $R$ pois a álgebra $R$ é prima. Daí,

$$
I x=\sum_{\beta} I q_{\beta}\left(1 \# h_{\beta}\right) \subseteq R\left(1 \# h_{\beta}\right) \subseteq R \# V
$$


(2) Pelo lema anterior, podemos escrever $x=\sum_{\beta} q_{\beta}\left(1 \# h_{\beta}\right)$, onde somente um número finito de $q_{\beta} \in Q_{l}$ é não nulo. Daí, $I x=\sum_{\beta} I q_{\beta}\left(1 \# h_{\beta}\right)=0$. Como $\left\{1 \# h_{\beta}\right\}$ é base do $Q_{l}$-módulo à esquerda $Q_{l} \# V, I q_{\beta}=0$, para todo $\beta$. Pelo Teorema 1.4.1, $q_{\beta}=0$, para todo $\beta$. Logo $x=0$.

(3) Seja $r \in I$. Como a dimensão de $V$ é finita, pelo lema anterior, podemos escrever $f(r)=$ $\sum_{i=1}^{n} r_{i}\left(1 \# h_{i}\right)$, onde $r_{i} \in Q_{l}$, para todo $1 \leq i \leq n$, de modo que $\left\{h_{i}\right\}_{1 \leq i \leq n}$ seja uma base de $V$.

Para todo $1 \leq i \leq n$, vamos definir $f_{i}=\pi_{i} \circ f: I \longrightarrow R$ por $f_{i}(r)=r_{i}$, para todo $r \in I$, que está bem definida pelo fato de $\left\{1 \# h_{i}\right\}_{1 \leq i \leq n}$ ser uma base para o $R$-módulo à esquerda $R \# V$. É fácil ver que $f_{i}$ é um homomorfismo de $R$-módulos à esquerda. Assim, pelo Teorema 1.4.1, existe $q_{i} \in Q_{l}$ tal que $f_{i}(r)=r q_{i}$, para todo $r \in I$. Seja $z=\sum_{i=1}^{n} q_{i} \# h_{i} \in Q_{l}$. Então, para todo $r \in I$,

$$
f(r)=\sum_{i=1}^{n} r_{i}\left(1 \# h_{i}\right)=\sum_{i=1}^{n} f_{i}(r)\left(1 \# h_{i}\right)=\sum_{i=1}^{n} r q_{i} \# h_{i}=r z
$$

(4) Pelo lema anterior, podemos escrever $x=\sum_{\beta}\left(1 \# h_{\beta}\right) q_{\beta}$, onde somente um número finito de $q_{\beta} \in Q_{l}$ é não nulo. Daí, $x I=\sum_{\beta}\left(1 \# h_{\beta}\right) q_{\beta} I=0$. Como $\left\{1 \# h_{\beta}\right\}$ é base do $Q_{l}$-módulo à direita $Q_{l} \# V, q_{\beta} I=0$, para todo $\beta$. Pelo Teorema 1.4.1, $q_{\beta}=0$, para todo $\beta$. Logo $x=0$.

(5) Como $J \subseteq R \subseteq Q_{l}$, basta mostrar que $\left(Q_{l} \# V\right)^{J} \subseteq\left(Q_{l} \# V\right)^{Q_{l}}$.

Seja $x \in\left(Q_{l} \# V\right)^{J}$ e seja $q \in Q_{l}$. Pelo Teorema 1.4.1, existe um $I \in \mathcal{F}(R)$ tal que $I q \subseteq R$. Assim, para todo $r \in J I \subseteq J$, temos

$$
r(q x-x q)=(r q) x-(r x) q=x(r q)-(x r) q=0,
$$

(Note que a penúltima igualdade decorre do fato que $J I q \subseteq J R \subseteq J$ ).

Logo, por (2), $x q=q x$, para todo $q \in Q_{l}$. Ou seja, $x \in\left(Q_{l} \# V\right)^{Q_{l}}$.

O próximo teorema, baseado em [Mil95a, Theorem 2.1], traz mais características a respeito dos elementos de $Q_{l} \# V$, onde $V$ é um coideal à esquerda de $H$ de dimensão finita.

Teorema 3.2.3. Seja $V$ um coideal à esquerda de $H$ de dimensão finita e seja $J$ um ideal não nulo de R. Sejam também $a \in Q_{l} \# V$ e $b \in Q_{l}$, onde b é não nulo. Então as seguintes condições são equivalentes: 
(i) $a \in b\left(Q_{l} \# V\right)^{J}$;

(ii) $a r b=b r a$, para todo $r \in Q_{l}$;

(iii) arb $=$ bra, para todo $r \in J$;

(iv) para todo $m \geq 1$ e todos $s_{j}, t_{j} \in R$, onde $1 \leq j \leq m$, se $\sum_{j=1}^{m} s_{j} b t_{j}=0$, então $\sum_{j=1}^{m} s_{j} a t_{j}=0$;

(v) para todo $m \geq 1$ e todos $s_{j}, t_{j} \in J$, onde $1 \leq j \leq m$, se $\sum_{j=1}^{m} s_{j} b t_{j}=0$, então $\sum_{j=1}^{m} s_{j} a t_{j}=0$.

Demonstração. (i) $\Rightarrow$ (ii): Seja $a=b z$, para algum $z \in\left(Q_{l} \# V\right)^{J}$. Pelo Lema 3.2.2 (5), temos que $z \in\left(Q_{l} \# V\right)^{Q_{l}}$. Assim,

$$
a r b=b z r b=b r z b=b r b z=b r a, \text { para todo } r \in Q_{l} .
$$

(ii) $\Rightarrow$ (iii): Trivial.

(iii) $\Rightarrow$ (iv): Temos que arb $=$ bra, para todo $r \in J$. Sejam $m \geq 1$ e $s_{j}, t_{j} \in R$, onde $1 \leq j \leq m$, tais que $\sum_{j=1}^{m} s_{j} b t_{j}=0$. Como $J R \subseteq J$, temos

$$
0=\sum_{j=1}^{m} a r s_{j} b t_{j}=\sum_{j=1}^{m} b r s_{j} a t_{j}=b r\left(\sum_{j=1}^{m} s_{j} a t_{j}\right), \text { para todo } r \in J
$$

Mas, pelo Teorema 1.4.1, existe $I \in \mathcal{F}(R)$ tal que $I b \subseteq R$. Assim, $(I b J)\left(\sum_{j=1}^{m} s_{j} a t_{j}\right)=0$, onde $I b J \in \mathcal{F}(R)$. Pelo Lema 3.2.2 (2), $\sum_{j=1}^{m} s_{j} a t_{j}=0$.

(iv) $\Rightarrow(\mathrm{v})$ : Trivial.

$(\mathrm{v}) \Rightarrow$ (i): Pelo Teorema 1.4.1, pelo Lema 3.2.2 (1) e pelo fato da álgebra $R$ ser prima, existe um $I \in \mathcal{F}(R)$ tal que $I b \subseteq R$ e $I a \subseteq R \# V$. Vamos definir

$$
\begin{array}{ccc}
f: & \text { JIbJ } & R \# V \\
\sum_{j=1}^{m} s_{j} b t_{j} & \longmapsto & \sum_{j=1}^{m} s_{j} a t_{j}
\end{array}
$$


Por (v), $f$ está bem definida e é fácil ver que é $(R, R)$-linear. Assim, pelo Lema 3.2.2 (3), existe $z \in Q_{l} \# V$ tal que $f(r)=r z$, para todo $r \in J I b J$. Vamos mostrar agora que $z \in\left(Q_{l} \# V\right)^{J}$ e que $a=b z$.

De fato, para todo $r \in J$ e $x \in J I b J$, temos

$$
x(r z-z r)=(x r) z-(x z) r=f(x r)-f(x) r=0 .
$$

Então $J \operatorname{IbJ}(r z-z r)=0$, para todo $r \in J$. Pelo Lema 3.2.2 (2), $z \in\left(Q_{l} \# V\right)^{J}$. Finalmente, para todo $s \in J I$ e todo $t \in J$, temos

$$
s(b z-a) t=s b z t-s a t=s b t z-f(s b t)=s b t z-s b t z=0 .
$$

Então $J I(b z-a) J=0$, portanto, pelo Lema 3.2.2 (2) e (4), $a=b z$.

O resultado seguinte, baseado em [Mil95a, Theorem 2.3], descreve uma propriedade importante dos $(R, R)$-sub-bimódulos de $Q_{l} \# H$.

Teorema 3.2.4. Seja $H$ uma álgebra de Hopf pontual de dimensão finita agindo em $R$, e seja $M$ um $(R, R)$-sub-bimódulo não nulo de $Q_{l} \# H$. Então, existem $g \in G(H), r \in R$ e $z \in\left(Q_{l} \# H\right)^{R}$ tais que $z(r \# g) \in M$ e $z(r \# g) \neq 0$.

Demonstração. Seja $\left\{H_{j}\right\}_{j \geq 0}$ a filtração co-radical de $H$ e seja $n$ o menor inteiro satisfazendo $M \cap$ $\left(Q_{l} \# H_{n}\right) \neq 0$. Se $x \neq 0$ é tal que $x \in M \cap\left(Q_{l} \# H_{n}\right)$, então, pelo Lema 3.2.2 (1) e (2), existe $s \in R$ tal que $s x \neq 0$ e $s x \in M \cap\left(R \# H_{n}\right)$. Daí $M \cap\left(R \# H_{n}\right) \neq 0$.

Seja $a \in M \cap\left(R \# H_{n}\right)$ não nulo. Pelo Teorema de Taft-Wilson (Teorema 2.3.1), podemos escrever $a=\sum_{i=1}^{m} b_{i} \# x_{i}+y$, onde $m \geq 1, b_{i} \in R, y \in R \# H_{n-1}$ e $x_{i} \in H_{n}$ são tais que $\Delta\left(x_{i}\right) \in g_{i} \otimes x_{i}+H \otimes H_{n-1}$, onde $g_{i} \in G(H)$, para todo $0 \leq i \leq m$. Vamos escolher um a para o qual $m$ seja minimal.

Como $M$ é um $(R, R)$-bimódulo e $g_{1}^{-1} \cdot\left(r b_{1}\right), b_{1} r \in R$, temos que $a\left(g_{1}^{-1} \cdot\left(r b_{1}\right)\right)-b_{1} r a \in M \cap\left(R \# H_{n}\right)$, 
para todo $r \in R$. Daí:

$$
\begin{aligned}
a\left(g_{1}^{-1} \cdot\left(r b_{1}\right)\right)-b_{1} r a & =\sum_{i=1}^{m} b_{i}\left[g_{i} \cdot\left(g_{1}^{-1} \cdot\left(r b_{1}\right)\right)\right] \# x_{i}-\sum_{i=1}^{m} b_{1} r b_{i} \# x_{i}+\bar{y}, \text { onde } \bar{y} \in R \# H_{n-1} \\
& =b_{1}\left[g_{1} \cdot\left(g_{1}^{-1} \cdot\left(r b_{1}\right)\right)\right] \# x_{1}+\sum_{i=2}^{m} b_{i}\left[g_{i} \cdot\left(g_{1}^{-1} \cdot\left(r b_{1}\right)\right)\right] \# x_{i} \\
& -b_{1} r b_{1} \# x_{1}-\sum_{i=2}^{m} b_{1} r b_{i} \# x_{i}+\bar{y} \\
& =\sum_{i=2}^{m}\left[b_{i}\left[g_{i} \cdot\left(g_{1}^{-1} \cdot\left(r b_{1}\right)\right)\right]-b_{1} r b_{i}\right] \# x_{i}+\bar{y}
\end{aligned}
$$

Ou seja,

$$
a\left(g_{1}^{-1} \cdot\left(r b_{1}\right)\right)-b_{1} r a=\sum_{i=1}^{m-1} c_{i} \# x_{i+1}+\bar{y}, \text { onde } c_{i}=b_{i+1}\left[g_{i+1} \cdot\left(g_{1}^{-1} \cdot\left(r b_{1}\right)\right)\right]-b_{1} r b_{i+1} \in R
$$

Pela minimalidade de $m$ e $n$, temos que $a\left(g_{1}^{-1} \cdot\left(r b_{1}\right)\right)-b_{1} r a=0$. Então

$$
a\left(1 \# g_{1}^{-1}\right) r b_{1}=a\left(g_{1}^{-1} \cdot\left(r b_{1}\right)\right) \# g_{1}^{-1}=b_{1} r a\left(1 \# g_{1}^{-1}\right), \text { para todo } r \in R \text {. }
$$

Assim, pelo Teorema 3.2.3,

$$
a\left(1 \# g_{1}^{-1}\right)=b_{1} z=z b_{1} \text {, para algum } z \in\left(Q_{l} \# H\right)^{R}
$$

Temos então que

$$
a=a\left(1 \# g_{1}^{-1}\right)\left(1 \# g_{1}\right)=z b_{1}\left(1 \# g_{1}\right)=z\left(b_{1} \# g_{1}\right) .
$$

Como, por hipótese $a \neq 0$, temos que $a=z\left(b_{1} \# g_{1}\right) \in M$ tem a propriedade desejada.

Observação. Da demonstração do teorema acima, podemos eliminar a hipótese da dimensão de $H$ ser finita. Para isso, basta considerar que $a \in H^{\prime}$, onde $H^{\prime}$ é uma subcoálgebra de $H$ de dimensão finita. O Teorema Fundamental das Coálgebras (Teorema 1.3.11) nos garante a existência de $H^{\prime}$. Porém, o teorema como está é suficiente para atender as necessidades deste trabalho. 


\subsection{Definição das ações X-externas}

O objetivo desta seção é obter uma série de resultados sobre a estrutura $(Q \# H)^{R}$ e definir quando uma ação de $H$ em $R$ é X-externa. Os resultados e definições aqui apresentados foram baseados em [Mil95a, Yan97].

Observe que o grupo $G=G(H)$ age em $R$ por automorfismos, ou seja, existe o seguinte homomorfismo de grupos

$$
\begin{array}{ccc}
G & \longrightarrow \quad \operatorname{Aut}(R) \\
\sigma & \longmapsto & (r \mapsto \sigma \cdot r),
\end{array}
$$

onde $\operatorname{Aut}(R)$ denota o grupo dos automorfismos da álgebra $R$. Definimos, para todo $\sigma \in G$,

$$
\Phi_{\sigma}=\{q \in Q: q(\sigma \cdot r)=r q, \text { para todo } r \in R\}
$$

Se $\Phi_{\sigma} \neq\{0\}$, dizemos que a ação de $\sigma$ em $R$ é $X$-interna, caso contrário dizemos que a ação de $\sigma$ em $R$ é $X$-externa. Se $\Phi_{\sigma}=\{0\}$, para todo $\sigma \in G \operatorname{com} \sigma \neq 1$, dizemos que a ação de $G$ em $R$ é $X$-externa.

Segue um resultado extraído de [Mil95a, Lemma 3.2].

Lema 3.3.1. Sejam $\sigma_{1}, \ldots, \sigma_{n}$ elementos de $G$ distintos dois a dois, e sejam $q_{1}, \ldots, q_{n} \in Q$. Então:

$$
\sum_{i=1}^{n} q_{i} \# \sigma_{i} \in(Q \# H)^{R} \text { se, e somente se, } q_{i} \in \Phi_{\sigma_{i}} \text { para todo } 1 \leq i \leq n \text {. }
$$

Demonstração. Seja $r \in R$. Observe que

$$
\begin{aligned}
r\left(\sum_{i=1}^{n} q_{i} \# \sigma_{i}\right) & =\sum_{i=1}^{n} r q_{i} \# \sigma_{i} \\
\left(\sum_{i=1}^{n} q_{i} \# \sigma_{i}\right) r & =\sum_{i=1}^{n} q_{i}\left(\sigma_{i} \cdot r\right) \# \sigma_{i} .
\end{aligned}
$$

Das expressões acima e do fato dos elementos group-like serem linearmente independentes, obtemos o resultado desejado.

Sejam $\sigma, \tau \in G$ e seja $\delta \in P_{\sigma, \tau}=P_{\sigma, \tau}(H)$. Observe que $\delta$ age como uma $(\sigma, \tau)$-derivação em 
$R$, ou seja, $\delta \cdot(r s)=(\sigma \cdot r)(\delta \cdot s)+(\delta \cdot r)(\tau \cdot s)$, para todos $r, s \in R$. Dizemos que a ação de $\delta$ em $R$ é $X$-interna ou dizemos que $\delta$ age como uma derivação $X$-interna se existir $q \in Q$ tal que $\delta \cdot r=(\sigma \cdot r) q-q(\tau \cdot r)$, para todo $r \in R$.

Sejam $\alpha \in K, \tau \in G$ e $\delta \in P_{1, \tau}$. Note que a aplicação linear

$$
\begin{array}{ccc}
R & \longrightarrow & Q \\
r & \longmapsto & (\delta \cdot r)
\end{array}
$$

é uma $(1, \tau)$-derivação. De fato,

$$
\alpha(\delta \cdot(r s))=\alpha[(1 \cdot r)(\delta \cdot s)+(\delta \cdot r)(\tau \cdot s)]=(1 \cdot r) \alpha(\delta \cdot s)+\alpha(\delta \cdot r)(\tau \cdot s), \text { para todos } r, s \in R
$$

Assim podemos definir o seguinte homomorfismo canônico

$$
\begin{array}{cl}
K \otimes P_{1, \tau} & \longrightarrow \operatorname{Der}_{1, \tau}(R, Q) \\
\alpha \otimes \delta & \longmapsto(r \mapsto \alpha(\delta \cdot r)),
\end{array}
$$

onde $\operatorname{Der}_{1, \tau}(R, Q)=\{f \in \operatorname{hom}(R, Q): f(r s)=r f(s)+f(r)(\tau \cdot s)$, para todos $r, s \in R\}$ denota o espaço vetorial de todas as $(1, \tau)$-derivações de $R$ em $Q$. Vamos denotar por $X_{i n n-D e r_{1, \tau}}(R, Q)$ o subespaço de $\operatorname{Der}_{1, \tau}(R, Q)$ de todas as $(1, \tau)$-derivações X-internas, ou seja,

$X_{\text {inn-Der }}(R, Q)=\left\{f \in \operatorname{Der}_{1, \tau}(R, Q):\right.$ existe $q \in Q$ tal que $f(r)=r q-q(\tau \cdot r)$, para todo $\left.r \in R\right\}$.

Da composição do homomorfismo acima com o homomorfismo canônico de $\operatorname{Der}_{1, \tau}(R, Q)$ em $\operatorname{Der}_{1, \tau}(R, Q) / X_{i n n-\operatorname{Der}_{1, \tau}}(R, Q)$, obtemos um homomorfismo

$$
\vartheta_{\tau}: K \otimes P_{1, \tau} \longrightarrow \operatorname{Der}_{1, \tau}(R, Q) / X_{i n n-D e r_{1, \tau}}(R, Q)
$$

Seja $\alpha \otimes(1-\tau) \in K \otimes P_{1, \tau}$. Vamos denotar $\vartheta_{\tau}(\alpha \otimes(1-\tau))=\omega$. Temos que

$$
\omega(r)=\alpha((1-\tau) \cdot r)=\alpha(1 \cdot r-\tau \cdot r)=r \alpha-\alpha(\tau \cdot r), \text { para todo } r \in R,
$$


ou seja, $\omega \in X_{\text {inn-Der } 1, \tau}(R, Q)$. Portanto, $K \otimes k(1-\tau) \subseteq \operatorname{ker}\left(\vartheta_{\tau}\right)$ e temos um homomorfismo

$$
\overline{\vartheta_{\tau}}: K \otimes P_{1, \tau}^{\prime} \longrightarrow \operatorname{Der}_{1, \tau}(R, Q) / X_{i n n-\operatorname{Der}_{1, \tau}}(R, Q),
$$

induzido por $\vartheta_{\tau}$ :

De [Mil95a, Proposition 3.4] temos o próximo resultado.

Proposição 3.3.2. As seguintes afirmações são equivalentes:

1. $(Q \# H)^{R}=K$;

2. $\overline{\vartheta_{\tau}}$ é injetora, para todo $\tau \in G$, e a ação de $G$ em $R$ é X-externa.

Demonstração. Se $(Q \# H)^{R}=K$, então é imediato que a ação de $G$ em $R$ é X-externa a partir do Lema 3.3.1. Seja $\tau \in G$. Se $\alpha_{1}, \ldots, \alpha_{n} \in K$ e $x_{1}, \ldots, x_{n} \in P_{1, \tau}$ são tais que $\sum_{i=1}^{n} \alpha_{i} \otimes x_{i} \in \operatorname{ker}\left(\vartheta_{\tau}\right)$, então existe $a \in Q$ tal que $\sum_{i=1}^{n} \alpha_{i}\left(x_{i} \cdot r\right)=r a-a(\tau \cdot r)$, para todo $r \in R$. Assim temos:

$$
\begin{aligned}
\left(\sum_{i=1}^{n} \alpha_{i} \# x_{i}+a \# \tau\right) r & =\sum_{i=1}^{n}\left(\alpha_{i} \# x_{i}\right) r+(a \# \tau) r \\
& =\sum_{i=1}^{n}\left[\alpha_{i} r \# x_{i}+\alpha_{i}\left(x_{i} \cdot r\right) \# \tau\right]+a(\tau \cdot r) \# \tau, \text { lembrar que } \Delta\left(x_{i}\right)=1 \otimes x_{i}+x_{i} \otimes \tau \\
& =\sum_{i=1}^{n} r \alpha_{i} \# x_{i}+\sum_{i=1}^{n} \alpha_{i}\left(x_{i} \cdot r\right) \# \tau+r a \# \tau-\sum_{i=1}^{n} \alpha_{i}\left(x_{i} \cdot r\right) \# \tau \\
& =r\left(\sum_{i=1}^{n} \alpha_{i} \# x_{i}+a \# \tau\right), \text { para todo } r \in R
\end{aligned}
$$

$\log 0 \sum_{i=1}^{n} \alpha_{i} \# x_{i}+a \# \tau \in(Q \# H)^{R}=K$. Note que $K \subseteq\left(Q \# H_{0}\right)^{R} \subseteq(Q \# H)^{R} \subseteq K$, ou seja, $\left(Q \# H_{0}\right)^{R}=(Q \# H)^{R}=K$. Como $a \otimes \tau \in Q \# H_{0}$, temos que

$$
\sum_{i=1}^{n} \alpha_{i} \otimes x_{i} \in\left(Q \otimes H_{0}\right) \cap\left(K \otimes P_{1, \tau}\right)=K \otimes\left(H_{0} \cap P_{1, \tau}\right)=K \otimes k(1-\tau),
$$


pela Proposição 1.3.1. Assim, concluímos que $\overline{\vartheta_{\tau}}$ é injetora para todo $\tau \in G$.

Se $\overline{\vartheta_{\tau}}$ é injetora para todo $\tau \in G$, e a ação de $G$ em $R$ é X-externa, basta mostrar que $\left(Q \# H_{n}\right)^{R}=$ $\left(Q \# H_{n-1}\right)^{R}$, para todo $n \geq 1$. Daí, pelo Lema 3.3.1 concluíremos que $(Q \# H)^{R}=K$.

Seja $n \geq 1$ e seja $x \in\left(Q \# H_{n}\right)^{R}$. Da Seção 2.3 , lembrar que $H_{n}=H_{n-1} \oplus X_{n}=H_{n-2} \oplus X_{n-1} \oplus X_{n}$. Assim, vamos escolher bases $\left\{x_{i}\right\}$ de $X_{n}$ e $\left\{x_{j}\right\}$ de $X_{n-1}$ tais que $x_{i} \in X_{\sigma_{i}, \tau_{i}, n}$, onde $\sigma_{i}, \tau_{i} \in G$, para todo $i$, e $x_{j} \in X_{\sigma_{j}, \tau_{j}, n-1}$, onde $\sigma_{j}, \tau_{j} \in G$, para todo $j$. Podemos escrever

$$
x=\sum_{i} q_{i} \# x_{i}+\sum_{j} q_{j} \# x_{j}+\widetilde{x}, \text { onde } q_{i}, q_{j} \in Q, \text { e } \widetilde{x} \in Q \# H_{n-2} .
$$

Pelo Corolário 2.3.6, temos que $\Delta\left(x_{i}\right)=\sigma_{i} \otimes x_{i}+\sum_{j} w_{i j} \otimes x_{j}+\widetilde{x_{i}}$, onde $\widetilde{x_{i}} \in H \otimes H_{n-2}$ e $w_{i j} \in X_{\sigma_{i}, \sigma_{j}, 1} \subseteq P_{\sigma_{i}, \sigma_{j}}$ (Proposição 2.3.2). Assim, para todo $r \in R$ temos que:

$$
\begin{aligned}
& x r \in \sum_{i}\left[q_{i}\left(\sigma_{i} \cdot r\right) \# x_{i}+\sum_{j} q_{i}\left(w_{i j} \cdot r\right) \# x_{j}\right]+\sum_{j} q_{j}\left(\sigma_{j} \cdot r\right) \# x_{j}+Q \# H_{n-2} \\
& r x \in \sum_{i} r q_{i} \# x_{i}+\sum_{j} r q_{j} \# x_{j}+Q \# H_{n-2}
\end{aligned}
$$

Como $x r=r x, q_{i}\left(\sigma_{i} \cdot r\right)=r q_{i}$, para todo $i$, ou seja, $q_{i} \in \Phi_{\sigma_{i}}$. Do fato da ação de $G$ em $R$ ser X-externa, temos que $\sigma_{i}=1$. Pelo Lema 3.3.1, $q_{i} \in K$, para todo $i$.

Temos também que $\sum_{i} q_{i}\left(w_{i j} \cdot r\right)=r q_{j}-q_{j}\left(\sigma_{j} \cdot r\right)$, para todo $j$, ou seja, a aplicação linear $\left(r \mapsto \sum_{i} q_{i}\left(w_{i j} \cdot r\right)\right)$ é uma $\left(1, \sigma_{j}\right)$-derivação X-interna. Assim $\sum_{i} q_{i} \otimes w_{i j} \in \operatorname{ker}\left(\vartheta_{\sigma_{j}}\right)$. Mas como todos $\overline{\vartheta_{\sigma_{j}}}$ são injetores, temos que $\operatorname{ker}\left(\vartheta_{\sigma_{j}}\right) \subseteq K \otimes H_{0}$. Logo:

$$
\begin{aligned}
(\operatorname{Id} \otimes \Delta)(x) & \in \sum_{i} q_{i} \otimes \sigma_{i} \otimes x_{i}+\sum_{j}\left(\sum_{i} q_{i} \otimes w_{i j}+q_{j} \otimes \sigma_{j}\right) \otimes y_{j}+Q \otimes H \otimes H_{n-2} \\
& \subseteq Q \otimes H_{0} \otimes H+Q \otimes H \otimes H_{n-2} \\
& =Q \otimes\left(H_{0} \otimes H+H \otimes H_{n-2}\right) .
\end{aligned}
$$

Daí, $x \in(\mathrm{Id} \otimes \Delta)^{-1}\left(Q \otimes\left(H_{0} \otimes H+H \otimes H_{n-2}\right)\right)=Q \otimes \Delta^{-1}\left(H_{0} \otimes H+H \otimes H_{n-2}\right)=Q \otimes H_{n-1}$, conforme desejado. 
A partir do fato de $R$ ser uma álgebra prima, é fácil verificar que $R^{\text {op }}$ também é uma álgebra prima. Note que $\left(Q_{r}\right)^{\mathrm{op}}=\left(Q^{\mathrm{op}}\right)_{l},\left(Q_{l}\right)^{\mathrm{op}}=\left(Q^{\mathrm{op}}\right)_{r}$, e que $(Q)^{\mathrm{op}}=Q^{\mathrm{op}}$, ou seja, a álgebra de quocientes de Martindale simétrica de $R^{\text {op }}$ coincide com a de $R$ como espaços vetoriais. Além disso, pelo fato de $K$ ser um corpo, $K^{\mathrm{op}}=K$.

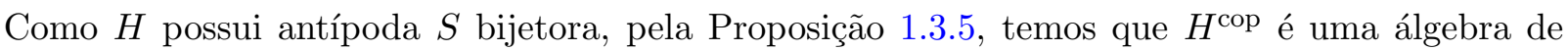
Hopf com antípoda $\bar{S}$. Assim como $H$, é fácil ver que $H^{\text {cop }}$ é álgebra de Hopf pontual. Seja $h \in H^{\text {cop }}$ e seja $r \in R^{\text {op }}$. Vamos definir $h \rightarrow r=h \cdot r$. Temos então, baseado em [Yan97, Proposition 2.6]:

Proposição 3.3.3. $R^{\mathrm{op}}$ é um $H^{\mathrm{cop}}$-módulo álgebra à esquerda com a ação "—".

Demonstração. É claro que $R^{\text {op }}$ é um $H^{\text {cop }}$-módulo à esquerda. Sejam $a, b \in R^{\text {op }}$ e seja $h \in H^{\text {cop }}$. Então:

$$
\begin{gathered}
h \rightarrow(a \bullet b)=h \cdot(b a)=\sum_{(h)}\left(h_{(1)} \cdot b\right)\left(h_{(2)} \cdot a\right)=\sum_{(h)}\left(h_{(2)} \rightarrow a\right) \bullet\left(h_{(1)} \rightarrow b\right) . \\
h \rightarrow 1_{R^{\mathrm{op}}}=h \cdot 1_{R^{\mathrm{op}}}=\varepsilon(h) 1_{R^{\mathrm{op}}}
\end{gathered}
$$

Observe que $1_{R^{\text {op }}}=1_{R}$ e que $\Delta^{\mathrm{cop}}(h)=(\tau \circ \Delta)(h)=\sum_{(h)} h_{(2)} \otimes h_{(1)}$. Assim $R^{\mathrm{op}}$ é um $H^{\mathrm{cop}}$-módulo álgebra à esquerda com a ação "—".

Como $H^{\text {cop }}$ é uma álgebra de Hopf pontual que age na álgebra prima $R^{\text {op }}$, valem todos os resultados mostrados para a ação de $H$ em $R$. O nosso próximo resultado foi baseado em [Yan97, Proposition 3.6].

Proposição 3.3.4. Temos:

1. $R^{H}=\left(R^{\mathrm{op}}\right)^{H^{\mathrm{cop}}}$.

2. As seguintes afirmações são equivalentes:

(i) $(Q \# H)^{R}=K$;

(ii) $\left(Q^{\mathrm{op}} \# H^{\mathrm{cop}}\right)^{R^{\mathrm{op}}}=K$.

Demonstração. (1) Aplicando o Lema 3.1.1, temos que:

$$
R^{H}=\{r \in R: h \cdot r=\varepsilon(h) r, \text { para todo } h \in H\}
$$




$$
\left(R^{\mathrm{op}}\right)^{H^{\mathrm{cop}}}=\left\{r \in R^{\mathrm{op}}: h \rightarrow r=\varepsilon(h) r, \text { para todo } h \in H^{\mathrm{cop}}\right\}
$$

A partir das expressões acima e do fato de $h \rightarrow r=h \cdot r$, para todo $h \in H$ e todo $r \in R$, concluímos que $R^{H}=\left(R^{\text {op }}\right)^{H^{\text {cop }}}$.

(2) Primeiramente, observe que $G=G(H)=G\left(H^{\text {cop }}\right)$, e que $\sigma \cdot \alpha \in K$, para todo $\sigma \in G$ e todo $\alpha \in K$. De fato,

$$
(\sigma \cdot \alpha) q=\sigma \cdot\left(\alpha\left(\sigma^{-1} \cdot q\right)\right)=\sigma \cdot\left(\left(\sigma^{-1} \cdot q\right) \alpha\right)=q(\sigma \cdot \alpha), \text { para todo } q \in Q .
$$

Se $(Q \# H)^{R}=K$, então a ação de $G$ em $R$ é X-externa pela Proposição 3.3.2. Vamos supor que exista $\sigma \in G$, com $\sigma \neq 1$, e $q \in Q^{\mathrm{op}}$, com $q \neq 0$, tais que $(\sigma \cdot r) q=q \bullet(\sigma \rightarrow r)=r \bullet q=q r$, para todo $r \in R$. Como $\sigma^{-1} \in G$ age como um automorfismo em $R$, podemos substituir $r$ por $\sigma^{-1} \cdot r$ e obter $\left(\sigma \cdot\left(\sigma^{-1} \cdot r\right)\right) q=q\left(\sigma^{-1} \cdot r\right)$, isto é, $r q=q\left(\sigma^{-1} \cdot r\right)$, para todo $r \in R$, um absurdo. Portanto, a ação de $G$ em $R^{\text {op }}$ é X-externa.

Ainda pela Proposição 3.3.2, temos que $\overline{\vartheta_{\tau}}$ é injetora para todo $\tau \in G$. Seja $\tau \in G$, e sejam $\alpha_{1}, \ldots, \alpha_{n} \in K$ e $\delta_{1}, \ldots, \delta_{n} \in P_{1, \tau}\left(H^{\text {cop }}\right)$ tais que $\sum_{i=1}^{n} \alpha_{i} \bullet\left(\delta_{i} \rightarrow r\right)=q \bullet(\tau \rightarrow r)-r \bullet q$, para algum $q \in Q^{\text {cop }}$ e para todo $r \in R$.

É claro que $\delta_{i} \in P_{\tau, 1}(H)$. Daí

$$
\Delta\left(\tau^{-1} \delta_{i}\right)=\Delta\left(\tau^{-1}\right) \Delta\left(\delta_{i}\right)=\left(\tau^{-1} \otimes \tau^{-1}\right)\left(\tau \otimes \delta_{i}+\delta_{i} \otimes 1\right)=1 \otimes \tau^{-1} \delta_{i}+\tau^{-1} \delta_{i} \otimes \tau^{-1},
$$

ou seja, $\tau^{-1} \delta_{i} \in P_{1, \tau^{-1}}(H)$, para todo $0 \leq i \leq n$. Temos ainda

$$
\begin{aligned}
\sum_{i=1}^{n}\left(\tau^{-1} \cdot \alpha_{i}\right)\left(\tau^{-1} \delta_{i} \cdot r\right) & =\tau^{-1} \cdot\left(\sum_{i=1}^{n} \alpha_{i} \bullet\left(\delta_{i} \rightarrow r\right)\right), \text { observe que } \alpha_{i} \in K \\
& =\tau^{-1} \cdot(q \bullet(\tau-r)-r \bullet q) \\
& =\tau^{-1} \cdot((\tau \cdot r) q-q r) \\
& =r\left(\tau^{-1} \cdot q\right)-\left(\tau^{-1} \cdot q\right)\left(\tau^{-1} \cdot r\right), \text { para todo } r \in R
\end{aligned}
$$

ou seja, $\sum_{i=1}^{n}\left(\tau^{-1} \cdot \alpha_{i}\right) \otimes \tau^{-1} \delta_{i} \in \operatorname{ker}\left(\vartheta_{\tau^{-1}}\right)=K \otimes\left(1-\tau^{-1}\right)$. Então, $\sum_{i=1}^{n} \alpha_{i} \otimes \delta_{i} \in(\tau \cdot K) \otimes \tau\left(1-\tau^{-1}\right) \subseteq$ $K \otimes k(1-\tau)$. Novamente pela Proposição 3.3.2, temos que $\left(Q^{\text {op }} \# H^{\text {cop }}\right)^{R^{\text {op }}}=K$. 
Se $\left(Q^{\mathrm{op}} \# H^{\mathrm{cop}}\right)^{R^{\mathrm{op}}}=K$, basta procedermos de forma análoga para concluirmos que $(Q \# H)^{R}=$ $K$.

Finalmente, vamos definir as ações X-externas.

Definição. A ação de $H$ em $R$ é chamada $X$-externa se $(Q \# H)^{R}=K$.

Observação. Essa definição foi primeiro sugerida por Milinski no artigo [Mil95a]. Milinski escolheu esse nome para homenagear Kharchenko, cujo nome em russo começa com a letra $X$. Kharchenko foi o autor de diversos trabalhos pioneiros no estudo de ações de grupos de automorfismos e de ações de álgebras de Lie restritas em álgebras primas e semi-primas. Alguns autores denominam as ações $X$-externas por ações $M$-externas, em homenagem a Milinski.

\subsection{Propriedades fundamentais das ações X-externas}

O objetivo desta seção é mostrar uma série de propriedades das ações X-externas. Essas propriedades serão fundamentais para os capítulos subsequentes. O primeiro resultado foi extraído de [Mil95a, Lemma 3.2].

Proposição 3.4.1. $\left(Q_{l} \# H\right)^{R} \subseteq Q \# H$.

Demonstração. Seja $z \in\left(Q_{l} \# H\right)^{R}$. Pelo Lema 3.2.1 (2), podemos escrever $z=\sum_{\beta}\left(1 \# h_{\beta}\right) q_{\beta}$, onde somente um número finito de $q_{\beta} \in Q_{l}$ é não nulo e $\left\{1 \# h_{\beta}\right\}$ é uma base para o $Q_{l}$-módulo à direita $Q_{l} \# H$. E, pelo Lema 3.2.1 (3), existe um $I \in \mathcal{F}(R)$ tal que $I z \subseteq R \# H$. Então temos:

$$
z I=I z \subseteq R \# H, \text { ou seja, } \sum_{\beta}\left(1 \# h_{\beta}\right) q_{\beta} I \subseteq R \# H
$$

Mas pelo Lema 3.2.1 (2), $\left\{1 \# h_{\beta}\right\}$ é também uma base para o $R$-módulo à direita $R \# H$. Assim $q_{\beta} I \subseteq R$, para todo $\beta$. Pela definição de $Q$, concluímos que $q_{\beta} \in Q$, para todo $\beta$. Logo, $z \in Q \# H$.

O seguinte resultado é consequência imediata da proposição anterior.

Corolário 3.4.2. Se a ação de $H$ em $R$ for X-externa, então

$$
\left(Q_{l} \# H\right)^{R}=K .
$$


Demonstração. Imediato a partir da proposição anterior.

Agora, vamos ver um dos resultados mais importantes de [Mil95a], o Theorem 4.1.

Teorema 3.4.3. Seja $H$ uma álgebra de Hopf pontual de dimensão finita agindo em $R$, e seja $I \in \mathcal{F}(R)$. Se a ação de $H$ em $R$ for X-externa, então a função

$$
\begin{array}{cc}
\Psi: \quad Q_{l} \otimes_{K}\left(Q_{l} \# H\right) & \longrightarrow \operatorname{hom}_{k}\left(I, Q_{l}\right) \\
q \otimes x & \longmapsto(r \mapsto(x \cdot r) q)
\end{array}
$$

é injetora.

Demonstração. Vamos supor que $\Psi$ não seja injetora e que $x=\sum_{i=1}^{n} q_{i} \otimes x_{i} \in \operatorname{ker}(\Psi)$, onde $n \geq 1$, $x_{1}, \ldots, x_{n} \in Q_{l} \# H, q_{1}, \ldots, q_{n} \in Q_{l}$ são $K$-linearmente independentes e $q_{i} \otimes x_{i} \neq 0$, para todo $1 \leq i \leq n$.

Será suficiente mostrar que existe $\sum_{i=1}^{n} q_{i} \otimes z_{i}(q \# g) \in \operatorname{ker}(\Psi)$ não nulo, onde $q \in Q_{l}, g \in G(H)$ e $z_{1}, \ldots, z_{n} \in\left(Q_{l} \# H\right)^{R}$, pois aí teríamos (Lembrar que, pelo Corolário 3.4.2, temos $\left(Q_{l} \# H\right)^{R}=K$.):

$$
\sum_{i=1}^{n} q_{i} \otimes z_{i}(q \# g)=\sum_{i=1}^{n} q_{i} z_{i} \otimes(q \# g)
$$

Daí $((q \# g) \cdot r) b=q(g \cdot r) b=0$, onde $b=\sum_{i=1}^{n} q_{i} z_{i}$, para todo $r \in I$. Pela continuidade da ação, existe $J \in \mathcal{F}(R)$ tal que $g^{-1} \cdot J \subseteq I$, e pelo Teorema 1.4.1, existe um $J^{\prime} \in \mathcal{F}(R)$ tal que $J^{\prime} q \subseteq R$. Logo $J^{\prime} q J b=0$. Como $q \neq 0$, temos que $J^{\prime} q J \in \mathcal{F}(R)$. Assim, pelo Lema 3.2.2 (2), $b=0$, uma contradição pois $q_{1}, \ldots, q_{n} \in Q_{l}$ são $K$-linearmente independentes.

Por indução em $n$, vamos mostrar que sempre chegaremos em uma contradição e concluir, portanto, que $\Psi$ é injetora.

Primeiramente, temos que $\sum_{i=1}^{n} q_{i} \otimes s x_{i} t \in \operatorname{ker}(\Psi)$, para todo $s, t \in R$. De fato, temos para todo 
$r \in I$,

$$
\begin{aligned}
\sum_{i=1}^{n}\left[\left(s x_{i} t\right) \cdot r\right] q_{i} & =\sum_{i=1}^{n}\left[\left(s x_{i}\right) \cdot(t \cdot r)\right] q_{i}, \text { identificando } t \text { com } t \# 1_{H} \\
& =\sum_{i=1}^{n}\left[s \cdot\left(x_{i} \cdot(t r)\right)\right] q_{i} \text {, identificando } s \text { com } s \# 1_{H} \\
& =\sum_{i=1}^{n}\left[s\left(x_{i} \cdot(t r)\right)\right] q_{i} \\
& =s\left[\sum_{i=1}^{n}\left(x_{i} \cdot(t r)\right) q_{i}\right] \\
& =0, \text { pois } t r \in I \text { e } \sum_{i=1}^{n} q_{i} \otimes x_{i} \in \operatorname{ker}(\Psi) .
\end{aligned}
$$

Assim, é fácil ver que

$$
M=\left\{y_{1} \in Q_{l}: \text { existem } y_{2}, \ldots, y_{n} \in Q_{l} \# H \text { tais que } \sum_{i=1}^{n} q_{i} \otimes y_{i} \in \operatorname{ker}(\Psi)\right\}
$$

é um $(R, R)$-sub-bimódulo de $Q_{l} \# H$ não nulo, já que $x_{1} \in M$. Pelo Teorema 3.2.4, existe $\widetilde{q} \in Q_{l}$, $\widetilde{g} \in G(H)$ e $\widetilde{z} \in\left(Q_{l} \# H\right)^{R}$, tal que $a_{1}=\widetilde{z}(\widetilde{q} \# \widetilde{g}) \in M$.

Vamos começar a indução. Para $n=1$, a contradição é imediata pois $q_{1} \otimes a_{1}=q_{1} \otimes \widetilde{z}(\widetilde{q} \# \widetilde{g}) \in$ $\operatorname{ker}(\Psi)$. Supondo válido para valores menores do que $n$, vamos mostrar que vale para $n$.

Pela definição de $M$, existem $a_{2}, \ldots, a_{n} \in Q_{l} \# H$ tais que $\sum_{i=1}^{n} q_{i} \otimes a_{i} \in \operatorname{ker}(\Psi)$. Como $a_{1}=$ $\widetilde{z}(\widetilde{q} \# \widetilde{g}) \in K(\widetilde{q} \# \widetilde{g})$, resta mostrar que $a_{i} \in K(\widetilde{q} \# \widetilde{g})$, para todo $2 \leq i \leq n$. Então, vamos supor que $a_{l} \notin K(\widetilde{q} \# \widetilde{g})$, para algum $2 \leq l \leq n$.

Assim, temos que $a_{l}\left(1 \# \widetilde{g}^{-1}\right) \notin K(\widetilde{q} \# \widetilde{g})\left(1 \# \widetilde{g}^{-1}\right)=K \widetilde{q}$. Portanto, pelo Teorema 3.2.3, existem $m \geq 1$ e $s_{j}, t_{j} \in R$, onde $1 \leq j \leq m$, tais que $\sum_{j=1}^{m} s_{j} \widetilde{q} t_{j}=0$ e $\sum_{j=1}^{m} s_{j} a_{l}\left(1 \# \widetilde{g}^{-1}\right) t_{j} \neq 0$. Vamos definir $u_{j}=\left(1 \# \widetilde{g}^{-1}\right) t_{j}(1 \# \widetilde{g})=\widetilde{g}^{-1} \cdot t_{j} \in R$, para todo $1 \leq j \leq m$. Então,

$$
\sum_{j=1}^{m} s_{j} a_{1} u_{j}=\sum_{j=1}^{m} s_{j} \widetilde{z}(\widetilde{q} \# \widetilde{g})\left(\widetilde{g}^{-1} \cdot t_{j}\right)=\sum_{j=1}^{m} s_{j} \widetilde{z}\left(\widetilde{q} t_{j} \# \widetilde{g}\right)=\widetilde{z}\left(\sum_{j=1}^{m} s_{j} \widetilde{q} t_{j}\right)(1 \# \widetilde{g})=0
$$


Além disso, $\sum_{j=1}^{m} s_{j} a_{l} u_{j}=\sum_{j=1}^{m} s_{j} a_{l}\left(1 \# \widetilde{g}^{-1}\right) t_{j}(1 \# \widetilde{g}) \neq 0$. De fato, supondo o contrário e multiplicando à direita por $1 \# \widetilde{g}^{-1}$, temos que $\sum_{j=1}^{m} s_{j} a_{l}\left(1 \# \widetilde{g}^{-1}\right) t_{j}=0$, uma contradição.

$\operatorname{Logo} \sum_{i=1}^{n}\left[q_{i} \otimes\left(\sum_{j=1}^{m} s_{j} a_{i} u_{j}\right)\right]=\sum_{i=2}^{n}\left[q_{i} \otimes\left(\sum_{j=1}^{m} s_{j} a_{i} u_{j}\right)\right] \in \operatorname{ker}(\Psi)$. Portanto, pela hipótese de indução, chegamos em uma contradição.

Observação. Como no Teorema 3.2.4, da demonstração do teorema acima, podemos também eliminar a hipótese da dimensão de $H$ ser finita. Para isso, basta perceber que podemos ver $a_{l} \in Q_{l} \# H^{\prime}$, onde $H^{\prime}$ é uma subcoálgebra de $H$ de dimensão finita. O Teorema Fundamental das Coálgebras (Teorema 1.3.11) nos garante a existência de $H^{\prime}$. Porém, o teorema como está é suficiente para atender as necessidades deste trabalho.

Finalmente, a proposição mais importante deste capítulo.

Proposição 3.4.4. Seja $H$ uma álgebra de Hopf pontual de dimensão finita agindo em $R$, e seja $U$ uma subálgebra de $R$ contendo $R^{H}$. Assuma que a ação de $H$ em $R$ é X-externa. Então:

1. Para todo $q \in Q_{l}$ não nulo, todo $\xi \in Q_{l} \# H$, e todo ideal $I \in \mathcal{F}(R)$, se $\xi \cdot(I q)=0$, então $\xi=0$

2. Seja $\xi \in Q_{l} \# H$ e seja $a \in Q_{l}$. Se $\xi U a=0$, então $\xi=0$ ou $a=0$. Em particular $U$ é uma álgebra prima.

3. Todo $(R, U)$-sub-bimódulo não nulo de $Q_{l}$ contém um ideal não nulo de $R$.

4. Seja $I \in \mathcal{F}(R)$ e $f: I \longrightarrow R \# H$, um homomorfismo de $R$-módulos à esquerda, então existe $z \in Q_{l} \# H$ tal que $f(r)=r z$, para todo $r \in I$.

5. $K$ é estável sob a ação de $H$, ou seja, $H \cdot K \subseteq K$.

6. $\left(Q_{l} \# H\right)^{U}=(Q \# H)^{U}=(K \# H)^{U}$.

7. $(K \# H)^{U}$ é um $H$-comódulo subálgebra à direita. 
Demonstração. Note que, como $H$ é uma álgebra de Hopf pontual de dimensão finita temos que a antípoda $S$ de $H$ é bijetora. Isso pode ser obtido tanto pelo Teorema 1.3 .18 quanto pelo Teorema 1.3.22.

(1) Baseado na demonstração de [Yan97, Lemma 3.5]:

Pelo Teorema de Taft-Wilson (Teorema 2.3.1) podemos escrever $\xi=\sum_{i=1}^{m} a_{i} \# h_{i}$, onde $a_{1}, \ldots, a_{m} \in$ $Q_{l}, h_{1}, \ldots, h_{m} \in H$ são $k$-linearmente independentes e, para todo $1 \leq i \leq m, h_{i}=\sigma_{i}$ ou $\Delta\left(h_{i}\right)=$ $h_{i} \otimes \sigma_{i}+\tau_{i} \otimes h_{i}+w_{i}$, onde $\sigma_{i}, \tau_{i} \in G(H)$ e $w_{i} \in H_{n_{i}-1} \otimes H_{n_{i}-1}$, sendo $n_{i}$ o grau de $h_{i}$.

Sejam $h_{1}, \ldots, h_{j}$ elementos de grau maximal $n$. Então,

$$
\begin{aligned}
0 & =\xi \cdot(I q)=\sum_{i=0}^{m}\left(a_{i} \# h_{i}\right) \cdot(I q)=\sum_{i=0}^{m} a_{i}\left(h_{i} \cdot(I q)\right) \\
& =\sum_{i=0}^{p} a_{i}\left(h_{i} \cdot I\right)\left(\sigma_{i} \cdot q\right)+\sum_{j} a_{j}^{\prime}\left(h_{j}^{\prime} \cdot I\right) q_{j}^{\prime}, \text { onde } a_{j}^{\prime}, q_{j}^{\prime} \in Q_{l} \text { e } h_{j}^{\prime} \in H_{n-1} .
\end{aligned}
$$

Note que $\sum_{i=0}^{p}\left(\sigma_{i} \cdot q\right) \otimes\left(a_{i} \# h_{i}\right)+\sum_{j} q_{j}^{\prime} \otimes\left(a_{j}^{\prime} \# h_{j}^{\prime}\right)$ está no núcleo da aplicação do Teorema 3.4.3, e portanto é igual a zero.

Como $h_{j}^{\prime} \in H_{n-1}$ e $h_{1}, \ldots, h_{p}$ são $k$-linearmente independentes de grau maximal $n$, temos que $\left(\sigma_{i} \cdot q\right) \otimes a_{i}=0$, para todo $1 \leq i \leq p$. Do fato de $q \neq 0$, temos que $\sigma_{i} \cdot q \neq 0$. De fato, se $\sigma_{i} \cdot q=0$, teríamos que $q=\left(\sigma_{i}^{-1} \sigma_{i}\right) \cdot q=\sigma_{i}^{-1} \cdot\left(\sigma_{i} \cdot q\right)=\sigma_{i}^{-1} \cdot 0=0$, um absurdo. Assim, concluímos que $a_{i}=0$, para todo $1 \leq i \leq p$.

Se $p<m$, podemos continuar o processo para $\xi^{\prime}=\sum_{i=p+1}^{m} a_{i} \# h_{i}$ e concluir facilmente que $a_{i}=0$, para todo $1 \leq i \leq m$. Logo $\xi=0$.

(2) Baseado na demonstração de [Mil95a, Theorem 4.3]:

Como $H$ tem dimensão finita, pelo Teorema 1.3.22, $H$ possui um integral à esquerda não nulo $t$, isto é, $t \in H$ e $h t=\varepsilon(h) t$, para todo $h \in H$. Temos que $t \cdot r \in R^{H}$, para todo $r \in R$. De fato,

$$
h \cdot(t \cdot r)=(h t) \cdot r=(\varepsilon(h) t) \cdot r=\varepsilon(h)(t \cdot r), \text { para todo } r \in R \text { e todo } h \in H \text {. }
$$


Então temos $\xi(t \cdot R) a=0$.

Pelo Lema $3.2 .1(2)$, podemos escrever $\xi=\sum_{\beta}\left(1 \# h_{\beta}\right) q_{\beta}$, onde $\left\{1 \# h_{\beta}\right\}$ é uma base para o $Q_{l}$-módulo à direita $Q_{l} \# H$. Daí temos que

$$
0=\xi(t \cdot R) a=\sum_{\beta}\left(1 \# h_{\beta}\right) q_{\beta}(t \cdot R) a
$$

Como $\left\{1 \# h_{\beta}\right\}$ é base para o $Q_{l}$-módulo à direita $Q_{l} \# H, q_{\beta}(t \cdot R) a=0$, para todo $\beta$. Note que $a \otimes\left(q_{\beta} \otimes t\right)$ está no núcleo da aplicação do Teorema 3.4.3, e portanto é igual a zero.

Como $t \neq 0$, temos que $a=0$ ou $q_{\beta}=0$, para todo $\beta$. Ou seja, temos que $a=0$ ou $\xi=0$. Portanto, em particular, $U$ é uma álgebra prima.

(3) Baseado em [Yan97, Lemma 4.3]:

Seja $M$ um $(R, U)$-sub-bimódulo de $Q_{l}$. Vamos mostrar que $M^{\prime}=(M \cap R) \subset M$, que é também um $(R, U)$-sub-bimódulo de $Q_{l}$, contém um ideal não nulo de $R$.

Como em (2), $H$ possui um integral à esquerda não nulo $t$. É fácil ver que $R(q \# t) R$ é um $(R, R)$-sub-bimódulo de $Q \# H$, para todo $q \in Q$ não nulo. Então, pelo Teorema 3.2.4, existem $r \in R, g \in G(H)$ e $\alpha \in(Q \# H)^{R}=K$ tais que $\alpha(r \# g) \in R(q \# t) R$, sendo $\alpha(r \# g) \neq 0$. Podemos escrever $\alpha(r \# g)=\sum_{i} r_{i}(q \# t) s_{i}$, onde $r_{i}, s_{i} \in R$, para todo $i$.

Temos que $\alpha(r \# g) I \neq 0$, para todo ideal $I \in \mathcal{F}(R)$. De fato, vamos supor que $\alpha(r \# g) I=0$. Como $\alpha \in K$, que é um corpo, temos

$$
0=(r \# g) I=r(g \cdot I) \# g=r(g \cdot I), \text { pois } g \neq 0 \text {. }
$$

Assim, $1 \otimes(r \# g)$ está no núcleo da aplicação do Teorema 3.4.3, e portanto é igual a zero. Logo $r \# g=0$, o que é um absurdo.

Assim, podemos escolher um $I \in \mathcal{F}(R)$ e um $a \in I$ tal que

$$
\sum_{i} r_{i}(q \# t) s_{i} a=\alpha(r \# g) a=\alpha r(g \cdot a) \# g \neq 0 .
$$

Como $\alpha r(g \cdot a) \in Q_{l}$, pelo Teorema 1.4.2, existe um $I^{\prime} \in \mathcal{F}(R)$ tal que $I^{\prime} \alpha r(g \cdot a) \subseteq R$ e $I^{\prime} \alpha r(g \cdot a) \neq 0$. 
Então temos que $\sum_{i} r_{i}^{\prime}(q \# t) s_{i}=a^{\prime} \# g \neq 0$, para algum $a^{\prime} \in I^{\prime} \alpha r(g \cdot a)$ e $r_{i}^{\prime} \in R$, para todo $i$.

Da continuidade da ação de $H$ em $R$, existe $I^{\prime \prime} \in \mathcal{F}(R)$ tal que $g^{-1} \cdot I^{\prime \prime} \subseteq I$. Para todo $x \in Q$ da forma $x=g^{-1} \cdot y$, onde $y \in I^{\prime \prime}$, temos

$$
\begin{gathered}
\left(a^{\prime} \# g\right) \cdot\left(g^{-1} \cdot y\right)=a^{\prime}\left(g \cdot\left(g^{-1} \cdot y\right)\right)=a^{\prime} y \\
\left.\sum_{i}\left[r_{i}^{\prime}(q \# t) s_{i}\right] \cdot\left(g^{-1} \cdot y\right)=\sum_{i,(t)}\left[r_{i}^{\prime} q\left(t_{(1)} \cdot s_{i}\right) \# t_{(2)}\right)\right] \cdot\left(g^{-1} \cdot y\right) \\
=\sum_{i,(t)} r_{i}^{\prime} q\left(t_{(1)} \cdot s_{i}\right)\left(t_{(2)} \cdot\left(g^{-1} \cdot y\right)\right) \\
=\sum_{i} r_{i}^{\prime} q\left(t \cdot\left(s_{i}\left(g^{-1} \cdot y\right)\right)\right) .
\end{gathered}
$$

Igualando as expressões e do fato de $t \cdot r \in R^{H}$, para todo $r \in R$, conforme mostrado em (2), temos que $a^{\prime} I^{\prime \prime} \subseteq R q R^{H}$. Lembrar que a escolha de $q \in Q$ não nulo foi aleatória.

Finalmente, seja $m \neq 0$ com $m \in M^{\prime} \subseteq R$. Pelos argumentos acima, existe algum $b \in R$ não nulo e algum ideal $J \in \mathcal{F}(R)$, tais que $R b J \subseteq R R m R^{H} \subseteq R m U \subseteq M^{\prime}$, pois $M^{\prime}$ é $(R, U)$-sub-bimódulo de $Q_{l}$. Note que $R b J$ é um ideal não nulo de $R$. De fato, caso $R b J=0$, pelo Teorema 1.4.1, $b=0$, um absurdo.

Assim, $M^{\prime}$ contém um ideal não nulo de $R$.

(4) Imediato a partir do Lema 3.2.2 (3).

(5) Baseado em [Mil95b, Bemerkung 15.3]:

Inicialmente vamos mostrar que se $z \in K=(Q \# H)^{R}$, então $z<h=\sum\left(1 \# S\left(h_{(1)}\right)\right) z\left(1 \# h_{(2)}\right) \in$ $K$, para todo $h \in H$. De fato, para todo $r \in R$, temos: 


$$
\begin{aligned}
(z<h) r & =\sum\left(1 \# S\left(h_{(1)}\right)\right) z\left(1 \# h_{(2)}\right) r \\
& =\sum\left(1 \# S\left(h_{(1)}\right)\right) z\left(h_{(2)} \cdot r \# h_{(3)}\right) \\
& =\sum\left(1 \# S\left(h_{(1)}\right)\right)\left(h_{(2)} \cdot r\right)\left(z \# h_{(3)}\right), \text { pois } z \in K . \\
& =\sum\left(S\left(h_{(1)}\right)_{(1)} \cdot\left(h_{(2)} \cdot r\right) \# S\left(h_{(1)}\right)_{(2)}\right)\left(z \# h_{(3)}\right) \\
& =\sum\left(S\left(h_{(2)}\right) \cdot\left(h_{(3)} \cdot r\right) \# S\left(h_{(1)}\right)\right)\left(z \# h_{(4)}\right), \text { pois } S \text { é antimorfismo de coálgebras. } \\
& =\sum\left(\left(S\left(h_{(2)}\right) h_{(3)}\right) \cdot r \# S\left(h_{(1)}\right)\right)\left(z \# h_{(4)}\right) \\
& =\sum\left(\varepsilon\left(h_{(2)}\right) r \# S\left(h_{(1)}\right)\right)\left(z \# h_{(3)}\right), \text { pois } \sum S\left(h_{(1)}\right) h_{(2)}=\varepsilon(h) 1_{H} . \\
& =\sum\left(r \# S\left(h_{(1)}\right)\right)\left(z \# h_{(2)}\right), \text { pois } \sum \varepsilon\left(h_{(1)}\right) h_{(2)}=h . \\
& =r\left[\sum\left(1 \# S\left(h_{(1)}\right)\right) z\left(1 \# h_{(2)}\right)\right] \\
& =r(z \leftarrow h) .
\end{aligned}
$$

Agora vamos mostrar que $S(h) \cdot z \in K$, para todo $h \in H$ e todo $z \in K$. De fato, inicialmente temos que,

$$
\begin{aligned}
z<h & =\sum\left(1 \# S\left(h_{(1)}\right)\right) z\left(1 \# h_{(2)}\right) \\
& =\sum\left(S\left(h_{(1)}\right)_{(1)} \cdot z \# S\left(h_{(1)}\right)_{(2)}\right)\left(1 \# h_{(2)}\right) \\
& =\sum\left(S\left(h_{(2)}\right) \cdot z \# S\left(h_{(1)}\right)\right)\left(1 \# h_{(3)}\right) \\
& =\sum S\left(h_{(2)}\right) \cdot z \# S\left(h_{(1)}\right) h_{(3)} .
\end{aligned}
$$

Como $z<h \in K$, temos que $(\operatorname{Id} \otimes \varepsilon)(z<h) \in K$, ou seja,

$$
\begin{aligned}
(\operatorname{Id} \otimes \varepsilon)(z \leftarrow h) & =(\operatorname{Id} \otimes \varepsilon)\left(\sum S\left(h_{(2)}\right) \cdot z \# S\left(h_{(1)}\right) h_{(3)}\right) \\
& =\sum\left(S\left(h_{(2)}\right) \cdot z\right) \varepsilon\left(S\left(h_{(1)}\right) h_{(3)}\right) \\
& =\sum\left(S\left(h_{(2)}\right) \cdot z\right) \varepsilon\left(S\left(h_{(1)}\right)\right) \varepsilon\left(h_{(3)}\right), \text { pois } \varepsilon \text { é morfismo de álgebras. } \\
& =\sum\left(\varepsilon\left(S\left(h_{(1)}\right)\right) S\left(h_{(2)}\right) \cdot z\right) \varepsilon\left(h_{(3)}\right) \\
& \left.=\sum\left(\varepsilon\left(S\left(h_{(1)}\right)_{(2)}\right) S\left(h_{(1)}\right)\right)_{(1)} \cdot z\right) \varepsilon\left(h_{(3)}\right) \\
& =\sum\left(S\left(h_{(1)}\right) \cdot z\right) \varepsilon\left(h_{(2)}\right) \\
& =\sum S\left(\varepsilon\left(h_{(2)}\right) h_{(1)}\right) \cdot z \\
& =S(h) \cdot z \in K .
\end{aligned}
$$


Como a antípoda $S$ de $H$ é bijetora, segue que $K$ é estável sob a ação de $H$.

(6) Baseado na demonstração de [Mil95a, Theorem 4.3]:

Como $(K \# H)^{U} \subseteq(Q \# H)^{U} \subseteq\left(Q_{l} \# H\right)^{U}$, para termos a igualdade basta mostrar que $\left(Q_{l} \# H\right)^{U} \subseteq$ $(K \# H)^{U}$. Como em (2), $H$ possui um integral à esquerda não nulo $t$ e $t \cdot r \in R^{H} \subseteq U$, para todo $r \in R$.

Seja $\xi \in\left(Q_{l} \# H\right)^{U}$, então $\xi(t \cdot r)-(t \cdot r) \xi=0$, para todo $r \in R$. Pelo Lema 3.2.1 (1), podemos escrever $\xi=\sum_{\beta} q_{\beta}\left(1 \# h_{\beta}\right)$, onde $q_{\beta} \in Q_{l}$, para todo $\beta$ e $\left\{1 \# h_{\beta}\right\}$ é uma base para o $Q_{l}$-módulo à esquerda $Q_{l} \# H$. Então temos:

$$
\begin{aligned}
0 & =\xi(t \cdot r)-(t \cdot r) \xi \\
& =\sum_{\beta} q_{\beta}\left(1 \# h_{\beta}\right)(t \cdot r)-(t \cdot r) \sum_{\beta} q_{\beta}\left(1 \# h_{\beta}\right) \\
& =\sum_{\beta}\left[q_{\beta}\left(\sum_{\left(h_{\beta}\right)} h_{\beta_{(1)}} \cdot(t \cdot r) \# h_{\beta_{(2)}}\right)-(t \cdot r) q_{\beta}\left(1 \# h_{\beta}\right)\right] \\
& =\sum_{\beta}\left[q_{\beta}\left(\sum_{\left(h_{\beta}\right)} \varepsilon\left(h_{\beta_{(1)}}\right)(t \cdot r) \# h_{\beta_{(2)}}\right)-(t \cdot r) q_{\beta}\left(1 \# h_{\beta}\right)\right], \text { Lema 3.1.1 aplicado em } t \cdot r \in R^{H} . \\
& =\sum_{\beta}\left[q_{\beta}(t \cdot r)\left(1 \# h_{\beta}\right)-(t \cdot r) q_{\beta}\left(1 \# h_{\beta}\right)\right] \\
& =\sum_{\beta}\left[q_{\beta}(t \cdot r)-(t \cdot r) q_{\beta}\right]\left(1 \# h_{\beta}\right) .
\end{aligned}
$$

Como $\left\{1 \# h_{\beta}\right\}$ é uma base para o $Q_{l}$-módulo à esquerda $Q_{l} \# H$, temos que $q_{\beta}(t \cdot r)-(t \cdot r) q_{\beta}=0$, para todo $\beta$. Então $1 \otimes\left(q_{\beta} \otimes t\right)-q_{\beta} \otimes(1 \otimes t)$ está no núcleo da aplicação do Teorema 3.4.3, e portanto, é igual a zero.

Assim, temos que $1 \otimes q_{\beta}=q_{\beta} \otimes 1$. Logo, $\left\{1, q_{\beta}\right\}$ é linearmente dependente sobre $K$, ou seja, existem $a, b \in K$ não nulos tais que $a+b q_{\beta}=0$. Então $q_{\beta}=-b^{-1} a \in K$, para todo $\beta$, o que implica em $\xi \in(K \# H)^{U}$.

(7) Baseado na demonstração de [Wes99, Lemma 1.3]:

É fácil ver que $(K \# H)^{U}$ é subálgebra de $Q \# H$. Falta verificar que $\rho\left((K \# H)^{U}\right) \subseteq(K \# H)^{U} \otimes H$. Seja $\xi=\sum q_{\alpha} \# h_{\alpha} \in(K \# H)^{U}$. Temos $\xi u=u \xi$, para todo $u \in U$. Então: 


$$
\begin{aligned}
& \rho(\xi)=\sum q_{\alpha} \# h_{\alpha_{(1)}} \otimes h_{\alpha_{(2)}}=\sum_{i=1}^{n} \xi_{i} \otimes h_{i}, \text { onde } \xi_{i} \in K \# H, \text { para todo } i . \\
& \xi u=\sum\left(q_{\alpha} \# h_{\alpha}\right) u=\sum q_{\alpha}\left(h_{\alpha_{(1)}} \cdot u\right) \# h_{\alpha_{(2)}}=\sum\left(q_{\alpha} \# h_{\alpha_{(1)}}\right) \cdot u \# h_{\alpha_{(2)}}=\sum_{i=1}^{n} \xi_{i} \cdot u \# h_{i} . \\
& u \xi=\sum u q_{\alpha} \# h_{\alpha}=\sum u \varepsilon\left(h_{\alpha_{(1)}}\right) q_{\alpha} \# h_{\alpha_{(2)}}=\sum_{i=1}^{n} u(\operatorname{Id} \otimes \varepsilon)\left(\xi_{i}\right) \# h_{i} .
\end{aligned}
$$

Como podemos considerar $\left\{h_{i}\right\}_{1 \leq i \leq n} k$-linearmente independente, temos que

$$
\xi_{i} \cdot u=u(\operatorname{Id} \otimes \varepsilon)\left(\xi_{i}\right) \text {, para todo } 1 \leq i \leq n \text { e todo } u \in U
$$

Seja $M=\operatorname{span}_{k}\left\{\xi_{i}\right\}_{1 \leq i \leq n}=\left\{\lambda_{1} \xi_{1}+\cdots+\lambda_{n} \xi_{n}: \lambda_{1}, \ldots, \lambda_{n} \in k\right\}$. Pelo Lema 1.3.9, $M$ é um $H$ subcomódulo à direita de $K \# H$, então $\rho\left(\xi_{i}\right)=\sum_{j=1}^{n} \xi_{j} \otimes l_{i j} \in M \otimes H$, onde $l_{i j} \in H$, para todo $1 \leq i, j \leq n$. Assim, temos que

$$
\xi_{i} u=\sum_{j=1}^{n} \xi_{j} \cdot u \# l_{i j}=\sum_{j=1}^{n} u(\operatorname{Id} \otimes \varepsilon)\left(\xi_{i}\right) \# l_{i j}=u \xi_{i}, \text { para todo } u \in U \text {. }
$$

Portanto, $\xi_{i} \in(K \# H)^{U}$, para todo $1 \leq i \leq n$, e $(K \# H)^{U}$ é uma $H$-comódulo subálgebra à direita. 


\section{Capítulo 4}

\section{A injetividade da correspondência de Galois}

\subsection{Introdução}

Finalmente, neste capítulo estamos em condições de mostrar a primeira parte da teoria da correspondência, objetivo deste trabalho. Os resultados aqui apresentados se baseiam principalmente no artigo [WY01].

No decorrer deste capítulo, $R$ será uma álgebra prima e $H$ será uma álgebra de Hopf pontual de dimensão finita agindo em $R$. Pela Proposição 3.4.4 (5) temos que $K$ é estável pela ação de $H$, então podemos construir o produto smash $K \# H$, que é uma $H$-comódulo-álgebra à direita via $\rho$ conforme vimos na Proposição 3.1.2.

Seja $U$ um subconjunto de $R$. Vamos definir

$$
\Phi(U)=(K \# H)^{U}
$$

Vamos definir também a aplicação linear $\varphi: Q \# H \longrightarrow Q^{\mathrm{op}} \# H^{\mathrm{cop}}$ dada por

$$
\varphi(a \# h)=\sum_{(h)} \bar{S}\left(h_{(1)}\right) \cdot a \# \bar{S}\left(h_{(2)}\right) \text {, para todo } a \in Q \text { e todo } h \in H .
$$


A proposição abaixo foi extraída de [WY01, Proposition 0.5].

Proposição 4.1.1. Temos:

1. A aplicação $\varphi$ é um antimorfismo bijetor de álgebras com inversa $\psi$ dada por

$$
\psi(a \# h)=\sum_{(h)} S\left(h_{(2)}\right) \cdot a \# S\left(h_{(1)}\right), \text { para todo } a \in Q^{\mathrm{op}} \text { e todo } h \in H^{\mathrm{cop}}
$$

2. Um conjunto $\Lambda$ é um $H$-comódulo subálgebra à direita de $Q \# H$ se, e somente se, $\varphi(\Lambda)$ for um $H^{\mathrm{cop}}$-comódulo subálgebra à direita de $Q^{\mathrm{op}} \# H^{\mathrm{cop}}$.

Demonstração. Sejam $a, b \in Q$ e sejam $h, l \in H$. Vamos denotar a multiplicação em $Q^{\text {op }} \# H^{\text {cop }}$ por "•". Inicialmente, observe que

$$
(a \# h) \bullet(b \# l)=a \bullet\left(h_{(2)} \cdot b\right) \# h_{(1)} l=\left(h_{(2)} \cdot b\right) a \# h_{(1)} l .
$$


Assim,

$\varphi((a \# h)(b \# l))$

$=\sum \varphi\left(a\left(h_{(1)} \cdot b\right) \# h_{(2)} l\right)$

$=\sum \bar{S}\left(\left(h_{(2)} l\right)_{(1)}\right) \cdot\left(a\left(h_{(1)} \cdot b\right)\right) \# \bar{S}\left(\left(h_{(2)} l\right)_{(2)}\right)$

$=\sum \bar{S}\left(h_{(2)} l_{(1)}\right) \cdot\left(a\left(h_{(1)} \cdot b\right)\right) \# \bar{S}\left(h_{(3)} l_{(2)}\right), \Delta$ é homomorfismo de álgebras.

$=\sum \bar{S}\left(l_{(1)}\right) \cdot\left[\bar{S}\left(h_{(2)}\right) \cdot\left(a\left(h_{(1)} \cdot b\right)\right)\right] \# \bar{S}\left(l_{(2)}\right) \bar{S}\left(h_{(3)}\right), \bar{S}$ é antimorfismo de álgebras.

$=\sum \bar{S}\left(l_{(1)}\right) \cdot\left[\left(\bar{S}\left(h_{(2)}\right)_{(1)} \cdot a\right)\left(\bar{S}\left(h_{(2)}\right)_{(2)} \cdot\left(h_{(1)} \cdot b\right)\right)\right] \# \bar{S}\left(l_{(2)}\right) \bar{S}\left(h_{(3)}\right)$

$=\sum \bar{S}\left(l_{(1)}\right) \cdot\left[\left(\bar{S}\left(h_{(3)}\right) \cdot a\right)\left(\left(\bar{S}\left(h_{(2)}\right) h_{(1)}\right) \cdot b\right)\right] \# \bar{S}\left(l_{(2)}\right) \bar{S}\left(h_{(4)}\right), \bar{S}$ é antimorfismo de coálgebras.

$=\sum \bar{S}\left(l_{(1)}\right) \cdot\left[\left(\bar{S}\left(h_{(1)}\right) \cdot a\right) b\right] \# \bar{S}\left(l_{(2)}\right) \bar{S}\left(h_{(2)}\right)$, pois $\sum \bar{S}\left(h_{(2)}\right) h_{(1)}=\varepsilon(h) 1_{H}$.

$=\sum\left(\bar{S}\left(l_{(1)}\right)_{(1)} \cdot\left(\bar{S}\left(h_{(1)}\right) \cdot a\right)\right)\left(\bar{S}\left(l_{(1)}\right)_{(2)} \cdot b\right) \# \bar{S}\left(l_{(2)}\right) \bar{S}\left(h_{(2)}\right)$

$=\sum\left(\bar{S}\left(l_{(2)}\right) \cdot\left(\bar{S}\left(h_{(1)}\right) \cdot a\right)\right)\left(\bar{S}\left(l_{(1)}\right) \cdot b\right) \# \bar{S}\left(l_{(3)}\right) \bar{S}\left(h_{(2)}\right), \bar{S}$ é antimorfismo de coálgebras.

$=\sum\left(\bar{S}\left(l_{(1)}\right) \cdot b\right) \bullet\left(\bar{S}\left(l_{(2)}\right) \cdot\left(\bar{S}\left(h_{(1)}\right) \cdot a\right)\right) \# \bar{S}\left(l_{(3)}\right) \bar{S}\left(h_{(2)}\right)$

$=\sum\left(\bar{S}\left(l_{(1)}\right) \cdot b\right) \bullet\left(\bar{S}\left(l_{(2)}\right)_{(2)} \cdot\left(\bar{S}\left(h_{(1)}\right) \cdot a\right)\right) \# \bar{S}\left(l_{(2)}\right)_{(1)} \bar{S}\left(h_{(2)}\right), \bar{S}$ é antimorfismo de coálgebras.

$=\sum\left(\bar{S}\left(l_{(1)}\right) \cdot b \# \bar{S}\left(l_{(2)}\right)\right) \bullet\left(\bar{S}\left(h_{(1)}\right) \cdot a \# \bar{S}\left(h_{(2)}\right)\right)$, por $(4.1)$.

$=\varphi(b \# l) \bullet \varphi(a \# h)$.

É fácil ver que $\varphi\left(1_{Q} \# 1_{H}\right)=1_{Q^{\text {op }}} \# 1_{H^{\text {cop }}}$. Assim mostramos que $\varphi$ é um antimorfismo de álgebras. Falta mostrar a bijeção. Temos:

$$
\begin{aligned}
\varphi(\psi(a \# h)) & =\sum \varphi\left(S\left(h_{(2)}\right) \cdot a \# S\left(h_{(1)}\right)\right) \\
& =\sum \bar{S}\left(S\left(h_{(1)}\right)_{(1)}\right) \cdot\left(S\left(h_{(2)}\right) \cdot a\right) \# \bar{S}\left(S\left(h_{(1)}\right)_{(2)}\right) \\
& =\sum \bar{S}\left(S\left(h_{(2)}\right)\right) \cdot\left(S\left(h_{(3)}\right) \cdot a\right) \# \bar{S}\left(S\left(h_{(1)}\right)\right), S \text { é antimorfismo de coálgebras. } \\
& =\sum h_{(2)} \cdot\left(S\left(h_{(3)}\right) \cdot a\right) \# h_{(1)} \\
& =\sum\left(h_{(2)} S\left(h_{(3)}\right)\right) \cdot a \# h_{(1)} \\
& =a \# h, \text { pois } \sum h_{(1)} S\left(h_{(2)}\right)=\varepsilon(h) 1_{H} .
\end{aligned}
$$


De forma análoga, temos $\psi(\varphi(a \# h))=a \# h$. Assim, $\varphi$ é um antimorfismo bijetor de álgebras com inversa $\psi$. Portanto, mostramos (1).

(2) Seja $\Lambda$ um $H$-comódulo subálgebra à direita de $Q \# H$. Por (1), temos que $\varphi(\Lambda)$ é subálgebra de $Q^{\mathrm{op}} \# H^{\mathrm{cop}}$. Vamos mostrar que $\varphi(\Lambda)$ é um $H^{\mathrm{cop}}$-comódulo subálgebra à direita de $Q^{\mathrm{op}} \# H^{\mathrm{cop}}$. Seja $\xi=\sum a_{i} \# h_{i} \in \Lambda$. Temos:

$$
\begin{aligned}
\left(\operatorname{Id}_{Q^{\mathrm{op}}} \otimes \Delta^{\mathrm{cop}}\right)(\varphi(\xi)) & =\left(\operatorname{Id}_{Q^{\mathrm{op}}} \otimes \Delta^{\mathrm{cop}}\right)\left(\sum \bar{S}\left(h_{i_{(1)}}\right) \cdot a_{i} \# \bar{S}\left(h_{i_{(2)}}\right)\right) \\
& =\sum \bar{S}\left(h_{i_{(1)}}\right) \cdot a_{i} \# \bar{S}\left(h_{i_{(2)}}\right)_{(2)} \otimes \bar{S}\left(h_{i_{(2)}}\right)_{(1)} \\
& =\sum \bar{S}\left(h_{i_{(1)}}\right) \cdot a_{i} \# \bar{S}\left(h_{i_{(2)}}\right) \otimes \bar{S}\left(h_{i_{(3)}}\right) \\
& =\sum \varphi\left(a_{i} \# h_{i_{(1)}}\right) \otimes \bar{S}\left(h_{i_{(2)}}\right) .
\end{aligned}
$$

Como $\Lambda$ é um $H$-comódulo subálgebra à direita, temos que $\left(\operatorname{Id}_{Q} \otimes \Delta\right)(\xi)=\sum a_{i} \# h_{i_{(1)}} \otimes h_{i_{(2)}} \in$ $\Lambda \otimes H$. Assim, $\left(\operatorname{Id}_{Q^{\text {op }}} \otimes \Delta^{\mathrm{cop}}\right)(\varphi(\xi))=\sum \varphi\left(a_{i} \# h_{i_{(1)}}\right) \otimes \bar{S}\left(h_{i_{(2)}}\right) \in \varphi(\Lambda) \otimes H^{\text {cop }}$. Então, concluímos que $\varphi(\Lambda)$ é um $H^{\mathrm{cop}}$-comódulo subálgebra à direita de $Q^{\mathrm{op}} \# H^{\mathrm{cop}}$.

A recíproca é mostrada de forma análoga.

\subsection{O teorema}

Esta seção se baseia no artigo [WY01] e tem por objetivo mostrar a primeira parte da teoria de correspôndencia entre subálgebras de $R$ que contém $R^{H}$, e $H$-comódulo subálgebras à direita de $K \# H$. Nesta seção vamos assumir que a ação de $H$ em $R$ é X-externa.

Inicialmente, vamos mostrar um resultado extraído de [WY01, Theorem 1.1].

Teorema 4.2.1. Seja $U$ uma subálgebra de $R$ contendo $R^{H}$ e seja $M$ um $(R \# H, U)$-sub-bimódulo não nulo de $Q \# H$. Então existe $I \in \mathcal{F}(R)$ e $\eta \in \Phi(U)$ satisfazendo $I \eta \subseteq M$, onde $I \eta \neq 0$.

Demonstração. Seja $\left\{H_{n}\right\}_{n \geq 0}$ a filtração co-radical de $H$ e seja $m$ o menor inteiro tal que $M \cap(Q \#$ $\left.H_{m}\right) \neq 0$. Da Seção 2.3, lembrar que $H_{m}=X_{m} \oplus H_{m-1}$. Daí, seja $\left\{x_{i}\right\}$ base de $X_{m}$ tal que $\Delta\left(x_{i}\right) \in \sigma_{i} \otimes x_{i}+H \otimes H_{m-1}$, onde $\sigma_{i} \in G(H)$, para todo $i$.

Dessa forma, podemos escrever um elemento $\xi \in Q \# H_{m}$ de forma única como $\xi=\sum\left(a_{i} \# x_{i}\right)+y$, 
onde $a_{i} \in Q$ e $y \in H_{m-1}$, para todo $i$. Seja $\xi \in M \cap\left(Q \# H_{m}\right)$ tal que $\xi \neq 0$ e o número de termos não nulos do somatório $\sum\left(a_{i} \# x_{i}\right)$ seja minimal. Vamos escrever $\xi=a_{1} \# x_{1}+\sum_{i \neq 1}\left(a_{i} \# x_{i}\right)+y$, onde $a_{1} \# x_{1} \neq 0$.

Seja

$$
\xi^{\prime}=\sigma_{1}^{-1} \xi=\sigma_{1}^{-1} \cdot a_{1} \# \sigma_{1}^{-1} x_{1}+\sum_{i \neq 1}\left(\sigma_{1}^{-1} \cdot a_{i} \# \sigma_{1}^{-1} x_{i}\right)+\sigma_{1}^{-1} y \in M,
$$

pois $M$ é $R \# H$-módulo à esquerda. Observe que $\xi^{\prime} \neq 0$. Vamos denotar $a=\sigma_{1}^{-1} \cdot a_{1}$. Como $M$ é um $(R \# H, U)$-sub-bimódulo, temos que $r \xi^{\prime} s \in M$, para todo $r \in R$ e todo $s \in U$. Além disso,

$$
r \xi^{\prime} s \in \operatorname{ras} \# \sigma_{1}^{-1} x_{1}+\sum_{i \neq 1} r\left(\sigma_{1}^{-1} \cdot a_{i}\right)\left(\sigma_{1}^{-1} \sigma_{i} \cdot s\right) \# \sigma_{1}^{-1} x_{i}+Q \# \sigma_{1}^{-1} H_{m-1} .
$$

Vamos mostrar que se $\sum r_{j} a s_{j}=0$, então $\sum r_{j} \xi^{\prime} s_{j}=0$, onde $r_{j} \in R$ e $s_{j} \in U$, para todo $j$. De fato, vamos supor que $\sum r_{j} \xi^{\prime} s_{j} \neq 0$. Seja $\xi^{\prime \prime}=\sigma_{1}\left(\sum r_{j} \xi^{\prime} s_{j}\right)=\sum b_{i} \# x_{i}+y^{\prime}$, onde $b_{i} \in Q$ e $y^{\prime} \in Q \# H_{m-1}$. É fácil ver que, ou $M \cap\left(Q \# H_{m-1}\right) \neq 0$, ou $\xi^{\prime \prime}$ teria menos elementos não nulos no somatório $\sum b_{i} \# x_{i}$ do que $\xi$. Em ambas as situações teríamos um absurdo.

Assim, está bem definida a aplicação linear

$$
\begin{aligned}
& f: \quad R a U \longrightarrow M \\
& \sum r_{j} a s_{j} \longmapsto \sum r_{j} \xi^{\prime} s_{j} .
\end{aligned}
$$

Pela Proposição 3.4.4 (2), $f$ é um homomorfismo de $R$-módulos à esquerda não nulo. Caso contrário, $R \xi^{\prime} U=0$, o que implicaria $\xi^{\prime}=0$. Um absurdo.

Pelo Lema 3.2.2 (1), pelo Teorema 1.4 .1 e pelo fato da álgebra $R$ ser prima, existe $J \in \mathcal{F}(R)$ tal que $J a U \subseteq R$ e $J \xi^{\prime} U \subseteq R \# H$. Como $J a U$ é um $(R, U)$-sub-bimódulo de $Q_{l}$, pela Proposição 3.4.4 (3), JaU contém um ideal $I \in \mathcal{F}(R)$.

Seja $f^{\prime}$ a restrição de $f$ com domínio $I$. Como podemos ver $f^{\prime}$ como um homomorfismo de $R$ módulos à esquerda, pela Proposição 3.4.4 (4), existe $\eta \in Q_{l}$ tal que $f(x)=x \eta$, para todo $x \in I$. Vamos mostrar que $\eta \neq 0$.

Vamos supor que $\eta=0$. Daí, para todo $x \in I$ e todo $y \in J a U \subseteq R$, temos que $0=x y \eta=$ $f(x y)=x f(y)$, ou seja, $I f(y)=0$. Pelo Lema 3.2.1 (4), temos que $f(y)=0$, para todo $y \in J a U$, 
o que implica em $J \xi^{\prime} U=0$. Pela Proposição 3.4.4 (2), temos que $J \xi^{\prime}=0$. Finalmente, pelo Lema 3.2.2 (2), temos que $\xi^{\prime}=0$, o que é um absurdo.

Seja $x=\sum r_{j} a s_{j} \in I$ e $s \in U$. Temos:

$$
x s \eta=\sum r_{j} a s_{j} s \eta=f\left(\sum r_{j} a s_{j} s\right)=\sum r_{j} \xi^{\prime} s_{j} s=f\left(\sum r_{j} a s_{j}\right) s=x \eta s .
$$

Assim, temos que $I(s \eta-\eta s)=0$. Novamente pelo Lema 3.2.2 (2), temos $s \eta=\eta s$, ou seja, $\eta \in$ $\left(Q_{l} \# H\right)^{U}$. Pela Proposição 3.4.4 (6), $\eta \in(K \# H)^{U}=\Phi(U)$.

Logo, existe $I \in \mathcal{F}(R)$ e $\eta \in \Phi(U)$ satisfazendo $I \eta \subseteq M$, onde $I \eta \neq 0$.

Da Seção 1.3.2, lembre que temos uma estrutura de $Q \# H$-módulo à esquerda e à direita em $Q$, dadas, respectivamente, por:

$$
\begin{aligned}
(a \# h) \cdot x & =a(h \cdot x), \text { para todos } a, x \in Q \text { e todo } h \in H . \\
x \triangleleft(a \# h) & =\bar{S}(h) \cdot(x a), \text { para todos } a, x \in Q \text { e todo } h \in H .
\end{aligned}
$$

Utilizaremos essas estruturas de $Q$ durante a demonstração do próximo lema, que foi extraído de [WY01, Lemma 1.2].

Lema 4.2.2. Seja $U$ uma subálgebra de $R$ contendo $R^{H}$. Então existe um ideal à direita não nulo de $R^{\Phi(U)}$ contido em $U$.

Demonstração. Como a dimensão de $H$ é finita, o Teorema 1.3 .22 nos garante a existência de um integral à esquerda não nulo $t \in H$. Pelo mesmo teorema, temos que $S(t)$ é um integral à direita. Da Seção 1.3.5, temos também que $h S(t)=\bar{\alpha}(h) S(t)$, para todo $h \in H$, onde $\alpha \in G\left(H^{*}\right)$ é o elemento group-like distinguido de $H^{*}$.

Seja $M=R S(t) U$. Para todo $r \# h \in R \# H$ e todo $u \in U$, temos que

$$
\begin{aligned}
(r \# h) M u & =(r \# h)((R \# S(t)) U) u \\
& \subseteq\left[\sum r\left(h_{(1)} \cdot R\right) \# h_{(2)} S(t)\right] U \\
& \subseteq\left[\sum \bar{\alpha}\left(h_{(2)}\right) r\left(h_{(1)} \cdot R\right) \# S(t)\right] U \\
& \subseteq(R \# S(t)) U=M .
\end{aligned}
$$


Assim, mostramos que $M$ é um $(R \# H, U)$-sub-bimódulo de $Q \# H$, e $M$ é não nulo já que $S(t) \in M$. Pelo Teorema 4.2.1, existe $I \in \mathcal{F}(R)$ e $\eta \in \Phi(U)$ satisfazendo $I \eta \subseteq M$, onde $I \eta \neq 0$. Vamos mostrar que $J=I \cap R^{\Phi(U)}$ é não nulo.

Como a ação de $H$ em $R$ é contínua, existe um ideal $I^{\prime} \in \mathcal{F}(R)$ tal que $t \cdot I^{\prime} \subseteq I$. Pela Proposição 3.4.4 (1), temos que $t \cdot I^{\prime} \neq 0$. Caso contrário, teríamos $t=0$, o que seria um absurdo. Além disso,

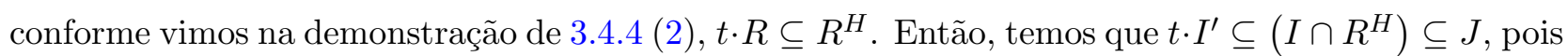
$R^{H} \subseteq R^{\Phi(U)}$. De fato, sejam $r_{i} \in K$ e $h_{i} \in H$, para todo $i$, tais que $\sum r_{i} \# h_{i} \in \Phi(U)=(K \# H)^{U}$. Para todo $v \in R^{H}$, temos que:

$$
\left(\sum r_{i} \# h_{i}\right) v=\sum r_{i}\left(h_{i_{(1)}} \cdot v\right) \# h_{i_{(2)}}=\sum r_{i}\left(\varepsilon\left(h_{i_{(1)}}\right) v\right) \# h_{i_{(2)}}=\sum r_{i} v \# h_{i}=v\left(\sum r_{i} \# h_{i}\right)
$$

É fácil ver que $R^{\Phi(U)}$ é subálgebra de $R$ e que $J$ é um ideal não nulo de $R^{\Phi(U)}$. Observe que, como $\eta \in \Phi(U)$ e $J=I \cap R^{\Phi(U)}$, temos que $J \eta=\eta J$. Além disso, como $J \subseteq I$ e $I \eta \subseteq M=R S(t) U$, temos $J \eta \subseteq R S(t) U$. Daí:

$$
\begin{aligned}
R \triangleleft(J \eta) & \subseteq R \triangleleft(R S(t) U) \\
& =R \triangleleft(R \# S(t)) U \\
& =R \triangleleft\left(\sum R\left(S(t)_{(1)} \cdot U\right) \# S(t)_{(2)}\right) \\
& =R \triangleleft\left(\sum R\left(S\left(t_{(2)}\right) \cdot U\right) \# S\left(t_{(1)}\right)\right), S \text { é antimorfismo de coálgebras. } \\
& =\sum \bar{S}\left(S\left(t_{(1)}\right)\right) \cdot\left[R R\left(S\left(t_{(2)}\right) \cdot U\right)\right] \\
& =\sum t_{(1)} \cdot\left[R\left(S\left(t_{(2)}\right) \cdot U\right)\right] \\
& =\sum\left(t_{(1)} \cdot R\right)\left(t_{(2)} \cdot\left(S\left(t_{(3)}\right) \cdot U\right)\right) \\
& =\sum\left(t_{(1)} \cdot R\right)\left(\left(t_{(2)} S\left(t_{(3)}\right)\right) \cdot U\right) \\
& =\sum\left(t_{(1)} \cdot R\right)\left(\left(\varepsilon\left(t_{(2)}\right) 1_{H}\right) \cdot U\right), \text { pois } \sum h_{(1)} S\left(h_{(2)}\right)=\varepsilon(h) 1_{H} . \\
& =(t \cdot R) U \\
& =U, \text { pois } t \cdot R \subseteq R^{H} \subseteq U .
\end{aligned}
$$


Podemos escrever $\eta=\sum r_{i} \# h_{i} \in \Phi(U)$. Então:

$$
\begin{aligned}
R \triangleleft(J \eta) & =R \triangleleft(\eta J)=R \triangleleft\left(\left(\sum r_{i} \# h_{i}\right) J\right)=R \triangleleft\left(\sum r_{i}\left(h_{i_{(1)}} \cdot J\right) \# h_{i_{(2)}}\right) \\
& =\sum \bar{S}\left(h_{i_{(2)}}\right) \cdot\left(R r_{i}\left(h_{i_{(1)}} \cdot J\right)\right) \\
& =\sum\left[\bar{S}\left(h_{i_{(2)}}\right)_{(1)} \cdot\left(R r_{i}\right)\right]\left[\bar{S}\left(h_{i_{(2)}}\right)_{(2)} \cdot\left(h_{i_{(1)}} \cdot J\right)\right] \\
& =\sum\left[\bar{S}\left(h_{i_{(3)}}\right) \cdot\left(R r_{i}\right)\right]\left[\left(\bar{S}\left(h_{i_{(2)}}\right) h_{i_{(1)}}\right) \cdot J\right], \bar{S} \text { é antimorfismo de coálgebras. } \\
& =\sum\left[\bar{S}\left(h_{i_{(2)}}\right) \cdot\left(R r_{i}\right)\right]\left[\left(\varepsilon\left(h_{i_{(1)}}\right) 1_{H}\right) \cdot J\right], \text { pois } \sum \bar{S}\left(h_{(2)}\right) h_{(1)}=\varepsilon(h) 1_{H} . \\
& =\sum\left[\bar{S}\left(h_{i}\right) \cdot\left(R r_{i}\right)\right] J \\
& =\left[R \triangleleft\left(\sum r_{i} \# h_{i}\right)\right] J=(R \triangleleft \eta) J .
\end{aligned}
$$

Segue que $R \triangleleft(J \eta)=(R \triangleleft \eta) J$ é um ideal à direita não nulo de $R^{\Phi(U)}$ contido em $U$. (Observe que $U \subseteq R^{\Phi(U)}$.)

Seja $U^{\text {op }}$ um sub-conjunto de $R^{\text {op }}$. Vamos definir

$$
\Phi^{\prime}\left(U^{\mathrm{op}}\right)=\left(K \# H^{\mathrm{cop}}\right)^{U^{\mathrm{op}}}
$$

Lembre que $K^{\mathrm{op}}=K$. O próximo lema, baseado em [WY01, Lemma 1.3], nos garante a existência de um ideal à esquerda não nulo de $R^{\Phi(U)}$ contido em $U$, onde $U$ é como no lema anterior. Basta aplicarmos o lema anterior em $\left(R^{\text {op }}\right)^{\Phi^{\prime}\left(U^{\text {op }}\right)}=\left(R^{\Phi(U)}\right)^{\text {op }}$, conforme veremos. Observe que $U^{\text {op }}$ é uma subálgebra de $R^{\mathrm{op}}$ contendo $\left(R^{\mathrm{op}}\right)^{H^{\text {cop }}}=R^{H}$, pela Proposição 3.3 .4 (1).

Lema 4.2.3. Seja $U$ uma subálgebra de $R$ contendo $R^{H}$. Então $\left(R^{\Phi(U)}\right)^{\mathrm{op}}=\left(R^{\mathrm{op}}\right)^{\Phi^{\prime}\left(U^{\mathrm{op}}\right)}$.

Demonstração. Seja $\varphi$ o antimorfismo bijetor de álgebras com inversa $\psi$ da Proposição 4.1.1. Seja $u \in Q$ e seja $\xi \in Q \# H$ tal que $u \xi=\xi u$. Temos:

$$
\varphi(\xi) \bullet u=\varphi(\xi) \bullet \varphi(u)=\varphi(u \xi)=\varphi(\xi u)=\varphi(u) \bullet \varphi(\xi)=u \bullet \varphi(\xi)
$$

Analogamente, se $u$ e $\xi$ são tais que $\xi \bullet u=u \bullet \xi$. Temos: 


$$
u \psi(\xi)=\psi(u) \psi(\xi)=\psi(\xi \bullet u)=\psi(u \bullet \xi)=\psi(\xi) \psi(u)=\psi(\xi) u
$$

Assim, temos que $u \xi=\xi u$ em $Q \# H$ se, e somente se, $\varphi(\xi) \bullet u=u \bullet \varphi(\xi)$ em $Q^{\text {op }} \# H^{\text {cop }}$. De forma análoga, temos que $u \bullet \xi=\xi \bullet u$ em $Q^{\text {op }} \# H^{\text {cop }}$ se, e somente se, $\psi(\xi) u=u \psi(\xi)$ em $Q \# H$.

Das seguintes definições

$$
\begin{gathered}
\Phi^{\prime}\left(U^{\mathrm{op}}\right)=\left(K \# H^{\mathrm{cop}}\right)^{U^{\mathrm{op}}}=\left\{\xi \in K \# H^{\mathrm{cop}}: \xi \bullet u=u \bullet \xi, \text { para todo } u \in U^{\mathrm{op}}\right\} \\
\Phi(U)=(K \# H)^{U}=\{\xi \in K \# H: \xi u=u \xi, \text { para todo } u \in U\}
\end{gathered}
$$

e das equações acima, temos que

$$
\Phi^{\prime}\left(U^{\mathrm{op}}\right)=(\varphi \circ \psi)\left(\Phi^{\prime}\left(U^{\mathrm{op}}\right)\right) \subseteq \varphi(\Phi(U)) \subseteq \Phi^{\prime}\left(U^{\mathrm{op}}\right), \text { ou seja, } \Phi^{\prime}\left(U^{\mathrm{op}}\right)=\varphi(\Phi(U))
$$

Seja $x \in\left(R^{\Phi(U)}\right)^{\text {op }}$. Para todo $\xi \in \Phi(U)$, temos que $\xi \bullet x=x \xi=\xi x=x \bullet \xi$. Da Equação (4.2), temos que $\varphi(\xi) \bullet x=x \bullet \varphi(\xi)$. Assim $x \in\left(R^{\mathrm{op}}\right)^{\varphi(\Phi(U))}=\left(R^{\mathrm{op}}\right)^{\Phi^{\prime}\left(U^{\mathrm{op}}\right)}$.

Seja $y \in\left(R^{\mathrm{op}}\right)^{\Phi^{\prime}\left(U^{\mathrm{op}}\right)}=\left(R^{\mathrm{op}}\right)^{\varphi(\Phi(U))}$. Para todo $\xi \in \Phi(U)$, temos que $\varphi(\xi) \bullet y=y \bullet \varphi(\xi)$. Da Equação (4.3), temos que $y \xi=y \psi(\varphi(\xi))=\psi(\varphi(\xi)) y=\xi y$. Assim, $y \in\left(R^{\Phi(U)}\right)^{\mathrm{op}}$.

Portanto, $\left(R^{\Phi(U)}\right)^{\mathrm{op}}=\left(R^{\mathrm{op}}\right)^{\Phi^{\prime}\left(U^{\mathrm{op}}\right)}$.

A seguir uma proposição extraída de [Yan05, Proposition 1.4] que trata das subálgebras de $R$ racionalmente completas, cuja definição está na Seção 1.2.

Proposição 4.2.4. Seja $U$ uma subálgebra de $R$ racionalmente completa e seja $U^{\prime}$ uma subálgebra de $R$ contendo $U$. Se $U$ contém um ideal $I$ não nulo de $U^{\prime}$, então $U=U^{\prime}$.

Demonstração. Seja $a \in U^{\prime}$. Observe que $I$ é um ideal não nulo de $U^{\prime}$ e $I \subseteq U$. Assim, temos que $a I \subseteq I \subseteq U$. Da definição de subálgebra racionalmente completa, concluímos que $a \in U$. Logo, $U=U^{\prime}$.

Finalmente, estamos em condições de mostrar o teorema que garante a injetividade da correspondência. O resultado foi extraído de [WY01, Theorem 1.4]. 
Teorema 4.2.5. Seja $R$ uma álgebra prima e seja $H$ uma álgebra de Hopf pontual de dimensão finita que age em $R$. Suponha que a ação de $H$ em $R$ é $X$-externa. Então a subálgebra $R^{\Phi(U)}$ possui um ideal não nulo contido em $U$, onde $U$ é qualquer subálgebra de $R$ contendo $R^{H}$.

Demonstração. Pelo Lema 4.2.2, existe um ideal à direita não nulo $A$ de $R^{\Phi(U)}$ contido em $U$.

Além disso, pela Proposição 3.3.3, temos que a álgebra de Hopf pontual de dimensão finita $H^{\text {cop }}$ age na álgebra prima $R^{\mathrm{op}}$. Pela Proposição 3.3.4 (2), a ação de $H^{\mathrm{cop}}$ em $R^{\mathrm{op}}$ é X-externa. É fácil ver que $U^{\text {op }}$ é subálgebra de $R^{\text {op }}$ contendo $\left(R^{\text {op }}\right)^{H^{\text {cop }}}=R^{H}$, pela Proposição 3.3 .4 (1). Logo, podemos aplicar o Lema 4.2.2 para $H^{\mathrm{cop}}, R^{\mathrm{op}}$ e $U^{\mathrm{op}}$ e obter um ideal à direita não nulo $B^{\mathrm{op}}$ de $\left(R^{\mathrm{op}}\right)^{\Phi^{\prime}\left(U^{\mathrm{op}}\right)}$ contido em $U^{\mathrm{op}}$.

Pelo Lema 4.2.3, temos que $\left(R^{\Phi(U)}\right)^{\mathrm{op}}=\left(R^{\mathrm{op}}\right)^{\Phi^{\prime}\left(U^{\mathrm{op}}\right)}$. Assim, podemos ver $B$ como um ideal à esquerda não nulo de $R^{\Phi(U)}$ contido em $U$.

Dessa forma, $B U A$ é um ideal de $R^{\Phi(U)}$ contido em $U$. Observe que, pela Proposição 3.4 .4 (2), temos que $B U A \neq 0$, caso contrário teríamos $A=0$ ou $B=0$, o que seria um absurdo.

Segue um colorário do teorema anterior que é imediato a partir da Proposição 4.2.4.

Corolário 4.2.6. Seja $R$ uma álgebra prima e seja $H$ uma álgebra de Hopf pontual de dimensão finita que age em $R$. Suponha que a ação de $H$ em $R$ é $X$-externa. Então $R^{\Phi(U)}=U$, onde $U$ é qualquer subálgebra de $R$ racionalmente completa contendo $R^{H}$. 


\section{Capítulo 5}

\section{A sobrejetividade da correspondência de Galois}

\subsection{Introdução}

Finalmente, estamos em condições de mostrar a sobrejetividade da correspondência, que é o principal objetivo deste capítulo. Os resultados principais aqui apresentados se baseiam no artigo [WY01].

No decorrer deste capítulo $R$ e $H$ serão como no capítulo anterior, ou seja, $R$ será uma álgebra prima e $H$ será uma álgebra de Hopf pontual de dimensão finita agindo em $R$. Além disso, vamos adicionar a hipótese da característica do corpo base $k$ ser igual a zero, e vamos assumir que a ação de $H$ em $R$ é X-externa.

Na Seção 5.2 mostramos uma série de propriedades da subálgebra $H^{K}$. A Seção 5.3 traz diversos resultados dos $H$-comódulo subálgebras de $K \# H$. Finalmente, na Seção 5.4, mostramos a sobrejetividade da correspondência de Galois. A Seção 5.5 finaliza este trabalho apresentando o enunciado completo do teorema da correspondência, como feito anteriormente na introdução. 


\subsection{Propriedades de $H^{K}$}

Esta seção se baseia no artigo [Wes00] e tem por objetivo mostrar uma série de propriedades da subálgebra $H^{K} \subseteq H$. Lembre que estamos identificando $K$ e $H$ com as subálgebras $K \# 1_{H}$ e $1_{K} \# H$ de $K \# H$, respectivamente.

Inicialmente, vamos mostrar um resultado extraído de [Wes00, Lemma 2.3].

Lema 5.2.1. Seja $\sigma \in G(H)$, seja $i>0$ e seja $h \in X_{1, \sigma, i}$, com $h \neq 0$. Então $L R(h) \subseteq H^{K}$.

Demonstração. Vamos mostrar o resultado por indução em $i$. Para $i=1$, temos que $h \in X_{1, \sigma, 1}$. Pela Proposição 2.3.2, $\Delta(h)=1 \otimes h+h \otimes \sigma$. Para todo $x \in K$ e todo $a \in R$, temos:

$$
\begin{aligned}
& h \cdot(x a)=x(h \cdot a)+(h \cdot x)(\sigma \cdot a) . \\
& h \cdot(a x)=a(h \cdot x)+(h \cdot a)(\sigma \cdot x) .
\end{aligned}
$$

Observe que $x a=a x$, e pela Proposição 3.4.4 (5), temos que $K$ é estável sob a ação de $H$. Assim, obtemos das expressões acima:

$$
(h \cdot a)(\sigma \cdot x-x)=(h \cdot x)(\sigma \cdot a-a) .
$$

Vamos supor que exista $x \in K$ tal que $\sigma \cdot x-x \neq 0$. Denotemos $\nu=\sigma \cdot x-x$, que é inversível pois $K$ é um corpo. Denotemos também $q=(h \cdot x) \nu^{-1}$. Multiplicando ambos os lados da igualdade acima por $\nu^{-1}$, obtemos:

$$
h \cdot a=q(\sigma \cdot a)-q a .
$$

Seja $\left\{H_{n}\right\}_{n \geq 0}$ a filtração co-radical de $H$. Como $H$ é álgebra de Hopf pontual, temos que $H_{0}=$ $k G(H)$. Além disso, $H_{1}=H_{0} \oplus X_{1}$. Então, temos que $h \in P_{1, \sigma}^{\prime}$. Seja $\overline{\vartheta_{\sigma}}$ a função da Proposição 3.3.2, que é injetora pois a ação de $H$ em $R$ é X-externa. Então:

$$
\begin{aligned}
\overline{\vartheta_{\sigma}}(q \otimes h)=\varphi: \quad R & \longrightarrow c \\
a & \longmapsto q(h \cdot a)
\end{aligned}
$$

Observe que $\varphi(a)=q(h \cdot a)=q(q(\sigma \cdot a)-q a)=q q(\sigma \cdot a)-q q a$, ou seja, $\varphi \in X_{i n n-D_{1, \sigma}}(R, Q)$. 
Assim, $q \otimes h \in \operatorname{ker} \overline{\vartheta_{\sigma}}$. Portanto, $q \otimes h=0$. Como $h \neq 0$, temos que $q=0$. Daí, $h \cdot R=0$. Considerando $\xi=1 \# h$ na Proposição 3.4.4 (1), é fácil ver que $h=0$, um absurdo. Logo, $\sigma$ age trivialmente em $K$. Pelo Lema 2.2.3, $\sigma \in H^{K}$.

Vamos supor que exista $x \in K$ tal que $h \cdot x \neq 0$. Denotemos $q=h \cdot x$. Da Equação 5.1, temos que $q(\sigma \cdot a)-a q=0$, para todo $a \in R$. Ou seja, a ação de $\sigma$ em $R$ é X-interna, conforme definido na Seção 3.3. Novamente pela Proposição 3.3.2, a ação de $G(H)$ em $R$ é X-externa, o que implica em $\sigma=1$. Daí, temos que $h \in X_{1,1,1}$. Pela Proposição $1.3 .2, h=0$, um absurdo. Logo, $h \cdot K=0$.

Lembre que, pela Proposição 2.3.4, $\varepsilon(h)=0$. Assim, $h$ age trivialmente em $K$. Pelo Lema 2.2.3, $h \in H^{K}$. Além disso, é fácil ver que $1_{H} \in H^{K}$. Como $L R(h)=\operatorname{span}_{k}\left\{1_{H}, \sigma, h\right\}$ e $H^{K}$ é subálgebra de $H$, temos que $L R(h) \subseteq H^{K}$.

Vamos assumir que o resultado seja válido para $i<n$. Seja $h \in X_{1, \sigma, n}$. Pelo Corolário 2.3.6, podemos escrever

$$
\Delta(h)=1 \otimes h+h \otimes \sigma+\sum x_{j} \otimes y_{j} \text {, onde } x_{j} \in \sum_{0<i<n} X_{1, \nu_{j}, i}, \text { e } y_{j} \in \sum_{0<i<n} X_{\nu_{j}, \sigma, i} \text {, para todo } j .
$$

Pela hipótese indutiva, temos que $L R\left(x_{j}\right) \subseteq H^{K}$. Em particular, temos que $\nu_{j} \in H^{K}$. Pela Proposição 2.3.3, temos que $\nu_{j}^{-1} y_{j} \in \sum_{0<i<n} X_{1, \nu_{j}^{-1} \sigma, i}$. Assim, pela hipótese indutiva, $L R\left(\nu_{j}^{-1} y_{j}\right) \in$ $H^{K}$. Pelo Lema 2.2.2, e pelo fato de $H^{K}$ ser uma subálgebra de $H$, temos que $\nu_{j} L R\left(\nu_{j}^{-1} y_{j}\right)=$ $L R\left(y_{j}\right) \in H^{K}$, para todo $j$. Em particular, observe que, como $y_{j} \in \sum_{0<i<n} X_{\nu_{j}, \sigma, i}$ e $L R\left(y_{j}\right) \in H^{K}$, temos que $\sigma \in H^{K}$. Além disso, é óbvio que $1 \in H^{K}$. Assim, falta mostrar que $h \in H^{K}$.

Para todo $x \in K$ e todo $a \in R$, temos:

$$
\begin{aligned}
& h \cdot(x a)=x(h \cdot a)+(h \cdot x)(\sigma \cdot a)+\sum\left(x_{j} \cdot x\right)\left(y_{j} \cdot a\right) . \\
& h \cdot(a x)=a(h \cdot x)+(h \cdot a)(\sigma \cdot x)+\sum\left(x_{j} \cdot a\right)\left(y_{j} \cdot x\right) .
\end{aligned}
$$

Mas, $\sigma, x_{j}, y_{j} \in H^{K}$. Pelo Lema 2.2.3, $\sigma, x_{j}$ e $y_{j}$ agem trivialmente em $K$, ou seja, $\sigma \cdot x=x$, $x_{j} \cdot x=y_{j} \cdot x=0$ (Lembre que, pela Proposição 2.3.4, $\varepsilon\left(X_{i}\right)=0$, para todo $i \geq 1$ ). Assim, a partir 
das expressões acima, temos a seguinte igualdade

$$
(h \cdot x)(\sigma \cdot a)=a(h \cdot x)
$$

Logo, podemos ter duas situações. Primeiro, podemos ter $h \cdot K=0$, ou seja, $h$ age trivialmente em $K$. Pelo Lema 2.2.3, $h \in H^{K}$.

Segundo, pode existir $x \in K$ tal que $h \cdot x \neq 0$. Daí, a ação de $\sigma$ em $R$ é X-interna, conforme definido na Seção 3.3. Novamente pela Proposição 3.3.2, a ação de $G(H)$ em $R$ é X-externa, o que implica em $\sigma=1$. Nesse caso, queremos chegar em uma contradição.

Como a dimensão de $H$ é finita, existe $m>0$ tal que $h^{m}=\sum_{i=1}^{m-1} \lambda_{i} h^{i}$, onde $\left\{h_{i}\right\}_{1 \leq i \leq m-1}$ é linearmente independente e $\lambda_{i} \in k$, para todo $1 \leq i \leq m-1$. Como $\sigma=1$, podemos escrever

$$
\Delta(h)=1 \otimes h+h \otimes 1+\sum x_{j} \otimes y_{j}
$$

Então, para todo $i \geq 1$, temos que

$$
\Delta\left(h^{i}\right)=1 \otimes h^{i}+h^{i} \otimes 1+\sum_{l=1}^{i-1}\left(\begin{array}{l}
i \\
l
\end{array}\right)\left(h^{l} \otimes h^{i-l}\right)+\sum a_{s_{i}} \otimes b_{s_{i}}
$$

onde $a_{i s}$ e $b_{i s}$ são monômios contendo, no mínimo, uma componente de $x_{j}$ e $y_{j}$, respectivamente.

Como $K$ é estável sob a ação de $H$ e a ação de $x_{j}$ e $y_{j}$ anulam $K$, temos que a ação de $a_{s_{i}}$ e $b_{s_{i}}$ anulam $K$. Temos ainda que:

$$
\begin{aligned}
\Delta\left(h^{m}\right) & =\sum_{i=1}^{m-1} \lambda_{i} \Delta\left(h^{i}\right)=\sum_{i=1}^{m-1} \lambda_{i}\left[1 \otimes h^{i}+h^{i} \otimes 1+\sum_{l=1}^{i-1}\left(\begin{array}{l}
i \\
l
\end{array}\right)\left(h^{l} \otimes h^{i-l}\right)+\sum a_{s_{i}} \otimes b_{s_{i}}\right] . \\
\Delta\left(h^{m}\right) & =1 \otimes h^{m}+h^{m} \otimes 1+\sum_{l=1}^{m-1}\left(\begin{array}{c}
m \\
l
\end{array}\right)\left(h^{l} \otimes h^{m-l}\right)+\sum a_{s_{m}} \otimes b_{s_{m}} \\
& =\sum_{i=1}^{m-1} \lambda_{i}\left[1 \otimes h^{i}+h^{i} \otimes 1\right]+\sum_{l=1}^{m-1}\left(\begin{array}{c}
m \\
l
\end{array}\right)\left(h^{l} \otimes h^{m-l}\right)+\sum a_{s_{m}} \otimes b_{s_{m}} .
\end{aligned}
$$


Igualando as expressões acima, obtemos

$$
\sum_{l=1}^{m-1}\left(\begin{array}{c}
m \\
l
\end{array}\right)\left(h^{l} \otimes h^{m-l}\right)+\sum a_{s_{m}} \otimes b_{s_{m}}=\sum_{i=1}^{m-1} \lambda_{i}\left[\sum_{l=1}^{i-1}\left(\begin{array}{l}
i \\
l
\end{array}\right)\left(h^{l} \otimes h^{i-l}\right)+\sum a_{s_{i}} \otimes b_{s_{i}}\right] .
$$

Seja $\Omega \in H^{*}$ tal que $\Omega\left(h^{t}\right)=0$, para todo $0<t<m-1$, e $\Omega\left(h^{m-1}\right)=1_{k}$. Observe que $\Omega$ existe já que podemos obter uma base de $H$ completando o conjunto linearmente independente $\left\{h_{i}\right\}_{1 \leq i \leq m-1}$. Aplicando $\operatorname{Id}_{H} \otimes \Omega$ na expressão acima, obtemos:

$$
h \otimes 1+\sum a_{s_{m}} \otimes \Omega\left(b_{s_{m}}\right)=\sum_{i=1}^{m-1} \lambda_{i}\left[\sum a_{s_{i}} \otimes \Omega\left(b_{s_{i}}\right)\right] .
$$

Então, temos que $h \in \operatorname{span}_{k}\left\{a_{s_{i}}\right\}_{1 \leq i \leq m}$. Como a ação de $a_{s_{i}}$ anula $K$, para todo $1 \leq i \leq m$, temos que $h \cdot K=0$, um absurdo.

Logo, $L R(h) \subseteq H^{K}$, para todo $\sigma \in G(H), i>0$ e $h \in X_{1, \sigma, i}$.

Segue um corolário do lema anterior, extraído de [Wes00, Corollary 2.4].

Corolário 5.2.2. Sejam $\sigma, \tau \in G(H)$ e seja $i>0$. Se $X_{\tau, \sigma, i} \neq\{0\}$, então:

(1) $L R\left(\tau^{-1} h\right) \subseteq H^{K}$, para todo $h \in X_{\tau, \sigma, i}$, com $h \neq 0$. Em particular, $\tau^{-1} \sigma \in H^{K}$.

(2) Se $\sigma \in H^{K}$, então $\tau \in H^{K}$ e $X_{\tau, \sigma, i} \subseteq H^{K}$.

Demonstração. (1) Pela Proposição 2.3.3, temos que $\tau^{-1} h \in X_{1, \tau^{-1} \sigma, i}$. Pelo Lema 5.2.1, $L R\left(\tau^{-1} h\right) \subseteq$ $H^{K}$. Em particular, é fácil ver que $\tau^{-1} \sigma \in L R\left(\tau^{-1} h\right) \subseteq H^{K}$.

(2) Pelo Lema 2.2.3, temos que $\sigma$ age trivialmente em $K$. Seja $x \in K$. Temos que

$$
x=\left(\sigma^{-1} \sigma\right) \cdot x=\sigma^{-1} \cdot(\sigma \cdot x)=\sigma^{-1} \cdot x
$$

ou seja, $\sigma^{-1}$ age trivialmente em $K$. Logo, $\sigma^{-1} \in H^{K}$.

De (1), temos que $\tau^{-1} \sigma \in H^{K}$. Daí, $\left(\tau^{-1} \sigma\right) \sigma^{-1}=\tau^{-1} \in H^{K}$. De forma análoga ao que foi feito para $\sigma$, temos $\left(\tau^{-1}\right)^{-1}=\tau \in H^{K}$. Novamente por (1), temos que $\tau^{-1} h \in H^{K}$. Assim, $\tau\left(\tau^{-1} h\right)=h \in H^{K}$. 
Logo, $X_{\tau, \sigma, i} \subseteq H^{K}$

Os dois próximos resultados foram extraídos de [Wes00, Theorem 2.5].

Proposição 5.2.3. $H^{K}$ é uma subálgebra de Hopf normal de $H$.

Demonstração. Pelo Lema 2.2.4, basta mostrar que $H^{K}$ é um coideal à esquerda de $H$ para concluir que $H^{K}$ é uma subálgebra de Hopf de $H$.

Seja $h \in H^{K}$. Pela Seção 2.3, podemos escrever $h=\sum_{i} \lambda_{i} \sigma_{i}+\sum_{i, j} h_{i, j}$, onde $\lambda_{i} \in k, h_{i, j} \in$ $X_{\sigma_{j}, \sigma_{i}, m_{i, j}}$, com $m_{i, j}>0$ e $\sigma_{i}, \sigma_{j} \in G(H)$, para todos $i, j$. Então, pelo Corolário 2.3.7, temos que

$$
\Delta(h)=\sum_{i} \lambda_{i}\left(\sigma_{i} \otimes \sigma_{i}\right)+\sum_{i, j} h_{i, j} \otimes \sigma_{i}+\sum_{l} a_{l} \otimes b_{l}
$$

onde $b_{l} \in \sum_{i>0} X_{i}$ e $\left\{b_{l}, \sigma_{i}\right\}_{l, i}$ é linearmente independente.

Pelo Lema 2.2.3, temos que $H^{K}$ é um coideal à direita de $H$. Segue que $\lambda_{i} \sigma_{i}+\sum_{j} h_{i, j} \in H^{K}$, para todo $i$. Lembre que, pela Proposição 2.3.4, $\varepsilon\left(X_{i}\right)=0$, para todo $i>0$. Assim, aplicando $\operatorname{Id} \otimes \varepsilon$ em $\Delta(h)$, temos que $h=\sum_{i}\left[\lambda_{i} \sigma_{i}+\sum_{j} h_{i, j}\right]$. Fixando um $i$, vamos denotar $\sigma=\sigma_{i}, \lambda=\lambda_{i}, h_{j}=h_{i, j}$, $m_{j}=m_{i, j}$, para todo $j$, e $h^{\prime}=\lambda \sigma+\sum_{j} h_{j}$. Observe que $h_{j} \in X_{\sigma_{j}, \sigma, m_{j}}, \operatorname{com} m_{j}>0$.

Pelo Corolário 2.3.6, temos que

$$
\Delta\left(h_{j}\right) \in \sigma_{j} \otimes h_{j}+h_{j} \otimes \sigma+\sum_{\nu \in G}\left[\sum_{\substack{0<z, w<m_{j} \\ z+w \leq m_{j}}}\left(X_{\sigma_{j}, \nu, z} \otimes X_{\nu, \sigma, w}\right)\right], \text { para todo } j .
$$

Se $\sigma \in H^{K}$, como $h^{\prime} \in H^{K}$, temos que $-\lambda \sigma+\lambda \sigma+\sum_{j} h_{j}=\sum_{j} h_{j} \in H^{K}$. Além disso, pelo Corolário 5.2.2, temos que $X_{\nu, \sigma, w} \subseteq H^{K}$, para todo $\nu \in G$, e todo $w>0$. Assim, a partir da expressão acima, e do fato de $h_{j} \in X_{\sigma_{j}, \sigma, m_{j}} \subseteq H^{K}$, para todo $j$, é fácil ver que $\Delta\left(h^{\prime}\right) \in H \otimes H^{K}$.

Logo, se $h^{\prime}=\lambda \sigma \in H^{K}$, então $\Delta\left(h^{\prime}\right) \in H \otimes H^{K}$. Vamos supor que $\sigma \notin H^{K}$. A partir de 
agora, vamos considerar somente os coeficientes $j$ para os quais $h_{j}$ é não nulo. Seja $m_{t}$ um elemento maximal no conjunto $\left\{m_{j}\right\}_{j}$. Então, temos:

$$
\begin{aligned}
\Delta\left(h^{\prime}\right) & =\lambda(\sigma \otimes \sigma)+\sum_{j} h_{j} \otimes \sigma+\sum_{\substack{j \\
j \neq t}} \sigma_{j} \otimes h_{j}+\sigma_{t} \otimes h_{t}+\sum_{z} a_{z} \otimes b_{z} \\
& =h^{\prime} \otimes \sigma+\sum_{\substack{j \\
j \neq t}} \sigma_{j} \otimes h_{j}+\sigma_{t} \otimes h_{t}+\sum_{z} a_{z} \otimes b_{z},
\end{aligned}
$$

onde $a_{z} \in H$ e $b_{z} \in X_{m_{z}}$, com $0<m_{z}<m_{t}$, para todo $z$.

Lembre que $H=H_{0} \oplus\left[\bigoplus_{n>0} X_{n}\right]$, onde $H_{0}=k G(H)$ é o co-radical de $H$. Além disso, da demonstração da Proposição 2.3.2, se $(\sigma, \tau, n) \neq\left(\sigma^{\prime}, \tau^{\prime}, n^{\prime}\right)$, então $X_{\sigma, \tau, n} \cap X_{\sigma^{\prime}, \tau^{\prime}, n^{\prime}}=\{0\}$, onde $\sigma, \sigma^{\prime}, \tau, \tau^{\prime} \in G(H)$ e $n, n^{\prime}>0$. Ainda, pelo fato de $H^{K}$ ser um coideal à direita de $H$, concluímos que $\sigma_{t} \in H^{K}$.

Do Corolário 5.2.2, $\sigma_{t}^{-1} \sigma \in H^{K}$. Como $H^{K}$ é subálgebra de $H, \sigma_{t}\left(\sigma_{t}^{-1} \sigma\right)=\sigma \in H^{K}$, um absurdo. Assim, $H^{K}$ é um coideal à esquerda de $H$.

Logo, conforme observação no início da demonstração, $H^{K}$ é uma subálgebra de Hopf de $H$. Falta mostrar a normalidade.

Seja $h \in H^{K}$, seja $y \in H$ e seja $x \in K$. Se $y=\tau \in G(H)$, então:

$$
\begin{aligned}
\left.\operatorname{ad}_{1}(\tau, h)\right] x & =\left(\tau h \tau^{-1}\right) x=(1 \# \tau)(1 \# h)\left(1 \# \tau^{-1}\right)(x \# 1) \\
& =(1 \# \tau)(1 \# h)\left(\tau^{-1} \cdot x \# \tau^{-1}\right) \\
& =(1 \# \tau)(1 \# h)\left(\tau^{-1} \cdot x\right)\left(1 \# \tau^{-1}\right) \\
& =(1 \# \tau)\left(\tau^{-1} \cdot x\right)(1 \# h)\left(1 \# \tau^{-1}\right), \text { pois } h \in H^{K} \text { e } H \cdot K \subseteq K . \\
& =\left(\tau \cdot\left(\tau^{-1} \cdot x\right) \# \tau\right)(1 \# h)\left(1 \# \tau^{-1}\right) \\
& \left.=x(1 \# \tau)(1 \# h)\left(1 \# \tau^{-1}\right)=x\left(\tau h \tau^{-1}\right)=x \operatorname{ad}_{l}(\tau, h)\right] .
\end{aligned}
$$

Então, $\operatorname{ad}_{1}(\tau, h) \in H^{K}$.

Se $y \in X_{\tau, \sigma, i}$, onde $i>0$ e $\tau, \sigma \in G(H)$, pelo Corolário 5.2.2, temos que $\tau^{-1} y \in H^{K}$. Como $H^{K}$ 
é uma subálgebra de Hopf de $H$, temos que

$$
\begin{aligned}
\operatorname{ad}_{l}\left(\tau^{-1} y, h\right) & =\sum_{(y)}\left(\tau^{-1} y\right)_{(1)} h S\left(\left(\tau^{-1} y\right)_{(2)}\right) \\
& =\sum_{(y)} \tau^{-1} y_{(1)} h S\left(\tau^{-1} y_{(2)}\right), \text { pois } \Delta \text { é morfismo de álgebras e } \Delta\left(\tau^{-1}\right)=\tau^{-1} \otimes \tau^{-1} . \\
& =\sum_{(y)} \tau^{-1} y_{(1)} h S\left(y_{(2)}\right) S\left(\tau^{-1}\right), \text { pois } S \text { é antimorfismo de álgebras. } \\
& =\sum_{(y)} \tau^{-1} y_{(1)} h S\left(y_{(2)}\right) \tau \in H^{K} .
\end{aligned}
$$

Mas,

$$
\operatorname{ad}_{l}\left(\tau, \operatorname{ad}_{l}\left(\tau^{-1} y, h\right)\right)=\tau \operatorname{ad}_{l}\left(\tau^{-1} y, h\right) \tau^{-1}=\tau\left[\sum_{(y)} \tau^{-1} y_{(1)} h S\left(y_{(2)}\right) \tau\right] \tau^{-1}=\sum_{(y)} y_{(1)} h S\left(y_{(2)}\right),
$$

ou seja, $\operatorname{ad}_{1}\left(\tau, \operatorname{ad}_{1}\left(\tau^{-1} y, h\right)\right)=\operatorname{ad}_{l}(y, h)$.

Conforme mostramos anteriormente, $\operatorname{ad}_{1}(\tau, h) \in H^{K}$, para todo $h \in H^{K}$ e todo $\tau \in G(H)$. Logo, $\operatorname{ad}_{l}(y, h) \in H^{K}$.

De forma análoga, mostra-se que $\operatorname{ad}_{\mathrm{r}}(y, h) \in H^{K}$, para todo $y \in H$ e todo $h \in H^{K}$. Portanto, $H^{K}$ é uma subálgebra de Hopf normal de $H$.

Observe que, pela proposição anterior, $H^{K}$ é uma subálgebra de Hopf normal de $H$. Daí, pelo Lema 1.3.6, $I=H\left(H^{K}\right)^{+}=\left(H^{K}\right)^{+} H$ é um ideal de Hopf. Sendo assim, o quociente $H / I$ é uma álgebra de Hopf com a estrutura induzida de $H$, e a projeção de $H$ em $H / I$, que vamos denotar por $\pi$, é um morfismo de álgebras de Hopf.

Denotemos por $\bar{H}$ a álgebra de Hopf $H / I=\pi(H)$, e por $\bar{G}=G(\bar{H})$ o grupo dos elementos group-like de $\bar{H}$. Seja $h \in H$. Denotemos por $\bar{h}$ a imagem de $h$ pela projeção $\pi$.

Finalmente, vamos mostrar o último resultado desta seção.

Proposição 5.2.4. Seja $\sigma \in G(H)$. Então:

(1) $\overline{X_{i}}=0$, para todo $i>0$. 
(2) $\bar{H}=k \bar{G}$, ou seja, $\bar{H}$ é uma álgebra de grupo.

(3) $\bar{\sigma} \neq 0$ e $\bar{\sigma} \in \bar{G}$.

(4) $\bar{\sigma}=\overline{1}$ se, e somente se, $\sigma \in G\left(H^{K}\right)$.

(5) $\bar{G}=G(H) / G\left(H^{K}\right)$.

Demonstração. Antes de mais nada, convém lembrar que, pela Proposição 2.3.4, $\varepsilon\left(X_{i}\right)=0$, para todo $i \geq 1$.

Seja $h \in X_{\sigma, \tau, i}$ não nulo, onde $\sigma, \tau \in G(H)$ e $i>0$. Pelo Corolário 5.2.2, temos que $\tau^{-1} h \in H^{K}$. Como $\varepsilon(h)=0$ e $\varepsilon$ é um morfismo de álgebras, segue que $\tau^{-1} h \in\left(H^{K}\right)^{+}$. Assim, $h=\tau \tau^{-1} h \in$ $H\left(H^{K}\right)^{+}=I$. Logo, temos que $\bar{h}=0$.

Pela Proposição 2.3.2, $X_{n}=\bigoplus_{\sigma, \tau \in G} X_{\sigma, \tau, n}$, para todo $n \geq 0$. Assim, temos que $\overline{X_{i}}=0$, para todo $i>0$. Logo, mostramos que vale (1).

(2) Da Seção 2.3, podemos escrever

$$
H=k G(H) \oplus\left[\bigoplus_{i>0} X_{i}\right] .
$$

Da expressão acima, concluímos que $\bar{H}=\overline{k G(H)}$. Do fato de $H$ ser pontual e do Teorema 1.3.17, temos que

$$
\bar{H}=\overline{k G(H)}=\overline{H_{0}}=\bar{H}_{0}=k G(\bar{H})=k \bar{G} .
$$

(3) Lembre que $\varepsilon$ é um morfismo de álgebras. Como $\varepsilon(\sigma)=1$ e $\varepsilon\left(H\left(H^{K}\right)^{+}\right)=\varepsilon(H) \varepsilon\left(\left(H^{K}\right)^{+}\right)=$ 0 , concluímos que $\bar{\sigma} \neq 0$. Além disso, observe que $\bar{\Delta} \circ \pi=(\pi \otimes \pi) \circ \Delta$, pois $\pi$ é morfismo de álgebras de Hopf. Dessa forma, é fácil ver que $\pi(\sigma) \in \bar{G}$.

(4) Se $\bar{\sigma}=\overline{1}$, temos que $\sigma-1 \in H\left(H^{K}\right)^{+}$. Pelo Lema 2.2.3, temos que $\left(H^{K}\right)^{+}$age trivialmente em $K$. Como $\left(H^{K}\right)^{+} \subseteq \operatorname{ker}(\varepsilon)$, temos que $\left(H^{K}\right)^{+} \cdot K=0$. Daí, $H\left(H^{K}\right)^{+} \cdot K=H \cdot\left(\left(H^{K}\right)^{+} \cdot K\right)=0$. Assim, $\sigma-1$ age trivialmente em $K$, o que implica que $\sigma$ age trivialmente em $K$. Novamente pelo Lema 2.2.3, $\sigma \in H^{K}$.

Se $\sigma \in G\left(H^{K}\right)$, como $1 \in H^{K}$ e $H^{K}$ é álgebra de Hopf, temos que $\sigma-1 \in\left(H^{K}\right)^{+} \subseteq H\left(H^{K}\right)^{+}$. Então, $\pi(\sigma-1)=0$, o que implica em $\pi(\sigma)=\bar{\sigma}=\pi(1)$. 
(5) Seja $\pi_{G(H)}: G(H) \longrightarrow \bar{G}$ a restrição de $\pi$ com domínio $G(H)$. Vamos mostrar que $\pi_{G(H)}$ é um epimorfismo de grupos, ou seja, que $\pi_{G(H)}$ é um morfismo sobrejetor de grupos.

Seja $\sigma^{\prime} \in \bar{G}$. De 5.2, existe $x=\sum_{\sigma \in G(H)} \lambda_{\sigma} \sigma \in H_{0}$ tal que $\pi(x)=\sigma^{\prime}$. Como $\pi$ é morfismo de álgebras de Hopf, podemos escrever

$$
(\bar{\Delta} \circ \pi)(x)=((\pi \otimes \pi) \circ \Delta)(x), \text { ou seja, } \sigma^{\prime} \otimes \sigma^{\prime}=\sum_{\sigma \in G(H)} \lambda_{\sigma}(\pi(\sigma) \otimes \pi(\sigma)) .
$$

Como $\pi(\sigma) \in \bar{G}$, para todo $\sigma \in G(H)$, concluímos que existe pelo menos um $\sigma \in G(H)$ tal que $\pi(\sigma)=\sigma^{\prime}$. Portanto, $\pi_{G(H)}$ é um epimorfismo de grupos.

Como $\pi(1)=\overline{1}$ é o elemento neutro de $\bar{G}$, observe que

$$
\operatorname{ker}\left(\pi_{G(H)}\right)=\{\sigma \in G(H): \pi(\sigma)=\pi(1)\}
$$

Do item (4), temos que $\operatorname{ker}\left(\pi_{G(H)}\right)=G\left(H^{K}\right)$. Logo, concluímos que $\bar{G}=G(H) / G\left(H^{K}\right)$.

\subsection{Propriedades dos comódulo subálgebras}

Esta seção se baseia no artigo [WY01] e tem por objetivo mostrar uma série de propriedades dos $H$-comódulo subálgebras de $K \# H$. No decorrer desta seção, vamos denotar $\Lambda$ um $H$-comódulo subálgebra de $K \# H$.

Proposição 5.3.1. $\Lambda$ é um $k \bar{G}$-comódulo à direita via $\bar{\rho}=\left(\operatorname{Id}_{K \# H} \otimes \pi\right) \circ \rho=\left(\operatorname{Id}_{K \# H} \otimes \pi\right) \circ\left(\operatorname{Id}_{K} \otimes \Delta\right)$.

Demonstração. Conforme vimos na seção anterior, $\pi$ é um morfismo de álgebras de Hopf. Assim, temos que $\bar{\Delta} \circ \pi=(\pi \otimes \pi) \circ \Delta$ e que $\bar{\varepsilon} \circ \pi=\varepsilon$.

Seja $\xi=\sum_{i} \alpha_{i} \# h_{i} \in \Lambda$. Temos que $\bar{\rho}(\xi)=\sum_{i}\left[\sum_{\left(h_{i}\right)} \alpha_{i} \# h_{i_{(1)}} \otimes \pi\left(h_{i_{(2)}}\right)\right]$. Então: 


$$
\begin{aligned}
\left(\left(\operatorname{Id}_{K \# H} \otimes \bar{\Delta}\right) \circ \bar{\rho}\right)(\xi) & =\sum_{i}\left[\sum_{\left(h_{i}\right)} \alpha_{i} \# h_{i_{(1)}} \otimes\left((\bar{\Delta} \circ \pi)\left(h_{i_{(2)}}\right)\right)\right] \\
& =\sum_{i}\left[\sum_{\left(h_{i}\right)} \alpha_{i} \# h_{i_{(1)}} \otimes\left(((\pi \otimes \pi) \circ \Delta)\left(h_{i_{(2)}}\right)\right)\right] \\
& =\sum_{i}\left[\sum_{\left(h_{i}\right)} \alpha_{i} \# h_{i_{(1)}} \otimes \pi\left(h_{i_{(2)}}\right) \otimes \pi\left(h_{i_{(3)}}\right)\right] \\
& =\left(\bar{\rho} \otimes \operatorname{Id}_{k \bar{G}}\right) \circ\left(\sum_{i}\left[\sum_{\left(h_{i}\right)} \alpha_{i} \# h_{i_{(1)}} \otimes \pi\left(h_{i_{(2)}}\right)\right]\right. \\
& =\left(\left(\bar{\rho} \otimes \operatorname{Id}_{k \bar{G}}\right) \circ \bar{\rho}\right)(\xi) . \\
\left(\left(\operatorname{Id}_{K \# H} \otimes \bar{\varepsilon}\right) \circ \bar{\rho}\right)(\xi) & =\sum_{i}\left[\sum_{\left(h_{i}\right)} \alpha_{i} \# h_{i_{(1)}} \otimes\left((\bar{\varepsilon} \circ \pi)\left(h_{i_{(2)}}\right)\right)\right] \\
& =\sum_{i}\left[\sum_{\left(h_{i}\right)} \alpha_{i} \# h_{i_{(1)}} \otimes \varepsilon\left(h_{i_{(2)}}\right)\right] \\
& =\sum_{i} \alpha_{i} \# h_{i} \otimes 1 \\
& =\xi \otimes 1 \\
&
\end{aligned}
$$

Logo, $\Lambda$ é um $k \bar{G}$-comódulo à direita via $\bar{\rho}$.

Observe que, pela proposição acima e pelo Exemplo 1.3.8, temos que $\Lambda$ é uma álgebra $\bar{G}$-graduada, e $\Lambda_{\bar{\sigma}}=\bar{\rho}^{-1}(\Lambda \otimes \bar{\sigma})$, para todo $\bar{\sigma} \in \bar{G}$.

A partir de agora, vamos denotar:

$$
G(\Lambda)=\Lambda \cap G(H) \quad \text { e } \quad \Lambda^{\prime}=\Lambda \cap\left(K \# H^{K}\right) .
$$

Observe que $\Lambda^{\prime}$ é um $H$-comódulo subálgebra de $K \# H$. Segue um resultado extraído de [WY01, Lemma 2.2]. 
Lema 5.3.2. Seja $\Lambda$ um $H$-comódulo subálgebra de $K \# H$ contendo $K$. Então:

1. Se $\sigma \in G(H)$, então $\Lambda_{\bar{\sigma}} \neq\{0\}$ se, e somente se, $G(\Lambda) \cap \Lambda_{\bar{\sigma}} \neq\{0\}$.

2. $\Lambda_{\overline{1}}=\Lambda^{\prime}$.

Demonstração. Seja $\xi=\sum \alpha_{i} \# h_{i} \in K \# H$. Observe que podemos fazer $h_{i}=\sigma_{i} \in G(H)$ ou $h_{i} \in X_{\tau_{i}, \sigma_{i}, n_{i}}$, onde $\sigma_{i}, \tau_{i} \in G(H)$ e $n_{i}>0$, para todo $i$, e $\left\{h_{i}\right\}$ é linearmente independente.

Lembre que $\Lambda_{\bar{\sigma}}=\bar{\rho}^{-1}(\Lambda \otimes \bar{\sigma})$, para todo $\bar{\sigma} \in \bar{G}$. Assim, pela Proposição 5.2 .4 (1) e pelo Corolário 2.3.7, é fácil ver que, se $\xi \neq 0$ e $\xi \in \Lambda_{\bar{\sigma}}$, então $\overline{\sigma_{i}}=\bar{\sigma}$, para todo $i$.

(1) Se $\xi \neq 0$ e $\xi \in \Lambda_{\bar{\sigma}}$, seja $n$ maximal tal que $\xi \in K \# H_{n}$ e $\xi \notin K \# H_{n-1}$.

Se $n=0$, então $\xi=\sum \alpha_{i} \# \sigma_{i}$. Como $\Lambda$ é um $H$-comódulo subálgebra, temos que $\sum \alpha_{i} \# \sigma_{i} \otimes \sigma_{i} \in$ $\Lambda \otimes H$. Como $\left\{\sigma_{i}\right\}$ é linearmente independente, temos que $\alpha_{i} \# \sigma_{i} \in \Lambda$, para todo $i$.

Do fato de $K$ ser um corpo, $K \subseteq \Lambda$ e $\Lambda$ ser uma subálgebra, temos que existe $\alpha_{i}^{-1} \in \Lambda$ e $\alpha_{i}^{-1}\left(\alpha_{i} \# \sigma_{i}\right)=\sigma_{i} \in \Lambda$. Além disso, temos que $\bar{\rho}\left(\sigma_{i}\right)=\sigma_{i} \otimes \bar{\sigma}$, ou seja, $\sigma_{i} \in G(\Lambda) \cap \Lambda_{\bar{\sigma}}$, para todo $i$. Como $\xi \neq 0$ e $\left\{\sigma_{i}\right\}$ é linearmente independente, temos que $\sigma_{i} \neq 0$, para todo $i$. Logo, $G(\Lambda) \cap \Lambda_{\bar{\sigma}} \neq\{0\}$.

Se $n>0$, podemos assumir que $\alpha_{1} \neq 0$ e que $h_{1} \in X_{\tau_{1}, \sigma_{1}, n}$. Pelo Corolário 2.3.7, temos que

$$
\Delta\left(h_{i}\right) \in \tau_{i} \otimes h_{i}+h_{i} \otimes \sigma_{i}+\sum_{\substack{0<j, p<n_{i} \\ j+p \leq n_{i}}} X_{j} \otimes X_{p}
$$

Pelo fato de $\Lambda$ ser um $H$-comódulo subálgebra, $n$ ser maximal e $\left\{h_{i}\right\}$ ser linearmente independente, podemos escrever

$$
\rho(\xi)=\left(\alpha_{1} \# \tau_{1}\right) \otimes h_{1}+\sum \xi_{j} \otimes y_{j} \in \Lambda \otimes H
$$

onde $\xi_{j} \otimes y_{j} \in \Lambda \otimes H$, para todo $j$, e $\left\{h_{1}\right\} \cup\left\{y_{j}\right\}$ é linearmente independente.

Então, temos que $\alpha_{1} \# \tau_{1} \in \Lambda$. Daí, conforme vimos anteriormente, $\tau_{1} \in \Lambda$. Pelo Corolário 5.2.2, temos que $\tau_{1}^{-1} \sigma_{1} \in H^{K}$. Pela Proposição 5.2.4, temos que

$$
\overline{1}=\pi(1)=\pi\left(\tau_{1}^{-1} \sigma_{1}\right)=\pi\left(\tau_{1}^{-1}\right) \pi\left(\sigma_{1}\right)=\pi\left(\tau_{1}^{-1}\right) \bar{\sigma} \text {, lembrar que } \overline{\sigma_{i}}=\bar{\sigma}, \text { para todo } i \text {. }
$$


Como $\pi(1)=\pi\left(\tau_{1}^{-1} \tau_{1}\right)=\pi\left(\tau_{1}^{-1}\right) \pi\left(\tau_{1}\right)$, da expressão acima, concluímos que $\pi\left(\tau_{1}\right)=\bar{\sigma}$. Assim, $\bar{\rho}\left(\tau_{1}\right)=\tau_{1} \otimes \bar{\sigma}$, ou seja, $\tau_{1} \in G(\Lambda) \cap \Lambda_{\bar{\sigma}}$. Logo, $G(\Lambda) \cap \Lambda_{\bar{\sigma}} \neq\{0\}$. É imediato que, se $G(\Lambda) \cap \Lambda_{\bar{\sigma}} \neq\{0\}$, então $\Lambda_{\bar{\sigma}} \neq\{0\}$.

(2) Se $\xi \in \Lambda^{\prime}=\Lambda \cap\left(K \# H^{K}\right)$, por (5.3) e pela Proposição 5.2.4, temos que $\bar{\rho}(\xi)=\sum \alpha_{i} \# h_{i} \otimes \overline{\sigma_{i}}$. Pela Proposição 5.2.3, temos que $H^{K}$ é subálgebra de Hopf. Assim, $\sigma_{i}, \tau_{i} \in H^{K}$, para todo $i$. Novamente pela Proposição 5.2.4, temos que $\overline{\sigma_{i}}=\overline{\tau_{i}}=\overline{1}$, para todo $i$. Daí, $\bar{\rho}(\xi)=\xi \otimes \overline{1}$ e $\Lambda^{\prime} \subseteq \Lambda_{1}$.

Se $\xi \in \Lambda_{1}$, então $\bar{\rho}(\xi)=\xi \otimes \overline{1}$. Assim, temos que $\overline{\sigma_{i}}=\overline{1}$, para todo $i$. Pela Proposição 5.2.4, $\sigma_{i} \in H^{K}$. Se $h_{i} \neq \sigma_{i}$, podemos aplicar o Corolário 5.2.2 e concluir que $X_{\tau_{i}, \sigma_{i}, i} \subseteq H^{K}$, para todo $i$. Como $\xi=\sum \alpha_{i} \# h_{i}$ e $h_{i}=\sigma_{i} \in H^{K}$ ou $h_{i} \in X_{\tau_{i}, \sigma_{i}, i} \subseteq H^{K}$, para todo $i$, concluímos que $\Lambda_{1} \subseteq \Lambda^{\prime}$.

Segue um resultado com mais informações a respeito dos $H$-comódulo subálgebras de $K \# H$ contendo $K$.

Lema 5.3.3. Seja $\Lambda$ um $H$-comódulo subálgebra de $K \# H$ contendo $K$. Então:

1. $\pi(G(\Lambda))=\overline{G(\Lambda)}$ é um subgrupo de $\bar{G}$. Além disso, $\overline{G(\Lambda)}=G(\Lambda) / G\left(\Lambda^{\prime}\right)$.

2. $\Lambda$ é uma álgebra fortemente $\overline{G(\Lambda)}$-graduada.

3. $\Lambda_{\overline{1}}$ é uma subálgebra de $\Lambda$.

4. Seja $\sigma \in G(\Lambda)$. Então $\Lambda_{\bar{\sigma}}=\Lambda_{\overline{1}} \sigma$.

5. Seja $\sigma \in G(\Lambda)$. Então $\Lambda_{\bar{\sigma}} \cong \Lambda_{\overline{1}} \otimes \bar{\sigma}$, tanto como $\Lambda_{\overline{1}}$-módulos à esquerda, quanto como $k \overline{G(\Lambda)}$ comódulos à direita.

Demonstração. (1) Como $\Lambda$ é subálgebra de $K \# H$, temos que $1_{H} \in G(\Lambda)$. Assim, temos que $\pi(1)=\overline{1} \in \overline{G(\Lambda)}$.

Sejam $\sigma, \tau \in G(\Lambda)$. Pelo fato de $\Lambda$ ser uma subálgebra de $K \# H$, temos que $\sigma \tau \in G(\Lambda)$. Além disso, observe que $\overline{\sigma \tau}=\bar{\sigma} \bar{\tau}$, pois $\pi$ é morfismo de álgebras de Hopf. Assim, se $\bar{\sigma}, \bar{\tau} \in \overline{G(\Lambda)}$, então $\bar{\sigma} \bar{\tau} \in \overline{G(\Lambda)}$.

Além disso, como $H$ tem dimensão finita e os elementos group-like de $H$ formam um conjunto linearmente independente, $G(H)$ é um grupo finito. Daí, $\sigma$ tem ordem finita, ou seja, existe $n \geq 1$ tal que $\sigma^{n}=1$. Se $n>1$, pelo fato de $\Lambda$ ser uma subálgebra de $K \# H$, temos que $\sigma^{n-1}=\sigma^{-1} \in G(\Lambda)$. 
Se $n=1$, temos que $\sigma=\sigma^{-1}=1 \in G(\Lambda)$. Observe que $\overline{1}=\overline{\sigma \sigma^{-1}}=\bar{\sigma} \overline{\sigma^{-1}}$, ou seja, $(\bar{\sigma})^{-1}=\overline{\sigma^{-1}}$. Assim, se $\bar{\sigma} \in \overline{G(\Lambda)}$, então $\overline{\sigma^{-1}}=(\bar{\sigma})^{-1} \in \overline{G(\Lambda)}$. Logo, $\overline{G(\Lambda)}$ é um subgrupo de $\bar{G}$.

Além disso, seja $\pi_{G(\Lambda)}: G(\Lambda) \longrightarrow \overline{G(\Lambda)}$ a restrição de $\pi$ com domínio $G(\Lambda)$. Pelo que vimos acima, temos que $\pi_{G(\Lambda)}$ é um epimorfismo de grupos. Da demonstração da Proposição 5.2.4 (5), sabemos que $\operatorname{ker}\left(\pi_{G(H)}\right)=G\left(H^{K}\right)$. Assim, temos que $\operatorname{ker}\left(\pi_{G(\Lambda)}\right) \subseteq G\left(H^{K}\right) \cap G(\Lambda)=G\left(\Lambda^{\prime}\right) . \operatorname{Logo}$, temos que $\overline{G(\Lambda)}=G(\Lambda) / G\left(\Lambda^{\prime}\right)$.

(2) Lembre que $\Lambda$ é uma álgebra $\bar{G}$-graduada, ou seja, $\Lambda=\bigoplus_{\bar{\sigma} \in \bar{G}} \Lambda_{\bar{\sigma}}$.

Pelo Lema 5.3.2, temos que $\Lambda_{\bar{\sigma}} \neq\{0\}$ se, e somente se, $G(\Lambda) \cap \Lambda_{\bar{\sigma}} \neq\{0\}$, para cada $\sigma \in G(H)$. Observe que, se $\tau \in G(\Lambda) \cap \Lambda_{\bar{\sigma}}$, então $\bar{\tau}=\bar{\sigma}$. Assim, é fácil ver que $\Lambda=\bigoplus_{\bar{\sigma} \in \overline{G(\Lambda)}} \Lambda_{\bar{\sigma}}$. Logo, $\Lambda$ é uma álgebra $\overline{G(\Lambda)}$-graduada. Falta mostrarmos que é fortemente graduada.

Sejam $\xi, \xi^{\prime} \in \Lambda$. Da definição de $H$-comódulo-álgebra, temos que $\rho\left(\xi \xi^{\prime}\right)=\rho(\xi) \rho\left(\xi^{\prime}\right)$. Além disso, $\pi$ é um morfismo de álgebras de Hopf. Assim, é fácil ver que $\bar{\rho}\left(\xi \xi^{\prime}\right)=\bar{\rho}(\xi) \bar{\rho}\left(\xi^{\prime}\right)$.

Sejam $\bar{\sigma}, \bar{\tau} \in \overline{G(\Lambda)}$ tal que $\Lambda_{\bar{\sigma}} \neq\{0\}$ e $\Lambda_{\bar{\tau}} \neq\{0\}$. Pelo Lema 5.3 .2 (1), podemos considerar $\xi \in \Lambda_{\bar{\sigma}}$ e $\xi^{\prime} \in \Lambda_{\bar{\tau}}$, ambos não nulos, tal que $\xi \xi^{\prime} \neq 0$. Lembre que $\bar{\rho}(\xi)=\xi \otimes \bar{\sigma}$ e que $\bar{\rho}\left(\xi^{\prime}\right)=\xi^{\prime} \otimes \bar{\tau}$. Então, temos que $\bar{\rho}\left(\xi \xi^{\prime}\right)=\bar{\rho}(\xi) \bar{\rho}\left(\xi^{\prime}\right)=(\xi \otimes \bar{\sigma})\left(\xi^{\prime} \otimes \bar{\tau}\right)=\xi \xi^{\prime} \otimes \bar{\sigma} \bar{\tau}$, ou seja, $\xi \xi^{\prime} \in \Lambda_{\bar{\sigma}} \bar{\tau}$. Portanto, $\Lambda_{\bar{\sigma}} \Lambda_{\bar{\tau}} \subseteq \Lambda_{\bar{\sigma} \bar{\tau}}$.

Dessa forma, temos que $\Lambda_{\overline{1}} \Lambda_{\bar{\sigma}} \subseteq \Lambda_{\bar{\sigma}}$. Como $1 \in \Lambda_{\overline{1}}$, temos também que $\Lambda_{\bar{\sigma}} \subseteq \Lambda_{\overline{1}} \Lambda_{\bar{\sigma}}$. Logo, $\Lambda_{\overline{1}} \Lambda_{\bar{\sigma}}=\Lambda_{\bar{\sigma}}$. De forma análoga, temos que $\Lambda_{\bar{\sigma}} \Lambda_{\overline{1}}=\Lambda_{\bar{\sigma}}$. Portanto, $\Lambda_{\bar{\sigma}} \Lambda_{\bar{\sigma}^{-1}}=\Lambda_{\bar{\sigma}^{-1}} \Lambda_{\bar{\sigma}}=\Lambda_{\overline{1}}$. Então:

$$
\Lambda_{\bar{\sigma} \bar{\tau}} \subseteq \Lambda_{\overline{1}} \Lambda_{\bar{\sigma} \bar{\tau}} \Lambda_{\overline{1}} \subseteq \Lambda_{\bar{\sigma}} \Lambda_{\bar{\sigma}^{-1}} \Lambda_{\bar{\sigma} \bar{\tau}} \Lambda_{\bar{\tau}^{-1}} \Lambda_{\bar{\tau}} \subseteq \Lambda_{\bar{\sigma}} \Lambda_{\overline{1}} \Lambda_{\bar{\tau}} \subseteq \Lambda_{\bar{\sigma}} \Lambda_{\bar{\tau}}
$$

Assim, mostramos que $\Lambda$ é uma álgebra fortemente $\overline{G(\Lambda)}$-graduada.

(3) Imediato a partir do Lema 5.3.2 (2).

(4) Seja $\xi \in \Lambda_{\bar{\sigma}}$. Observe que $\bar{\rho}\left(\xi \sigma^{-1}\right)=\bar{\rho}(\xi) \bar{\rho}\left(\sigma^{-1}\right)=(\xi \otimes \bar{\sigma})\left(\sigma^{-1} \otimes \bar{\sigma}^{-1}\right)=\xi \sigma^{-1} \otimes \bar{\sigma} \bar{\sigma}^{-1}=$ $\xi \sigma^{-1} \otimes \overline{1}$, ou seja, $\xi \sigma^{-1} \in \Lambda_{\overline{1}}$. Assim, temos $\Lambda_{\bar{\sigma}}=\Lambda_{\bar{\sigma}} \sigma^{-1} \sigma \subseteq \Lambda_{\overline{1}} \sigma \subseteq \Lambda_{\overline{1}} \Lambda_{\bar{\sigma}}=\Lambda_{\bar{\sigma}}$. Logo, $\Lambda_{\bar{\sigma}}=\Lambda_{\overline{1}} \sigma$. 
(5) Observe que ambos $\Lambda_{\overline{1}} \sigma$ e $\Lambda_{\overline{1}} \otimes \bar{\sigma}$ têm estrutura trivial de $\Lambda_{\overline{1}}$-módulos à esquerda. Considere

$$
\begin{aligned}
f: \Lambda_{\bar{\sigma}}=\Lambda_{\overline{1}} \sigma & \longrightarrow \Lambda_{\overline{1}} \otimes \bar{\sigma} \\
\xi \sigma & \longmapsto \xi \otimes \bar{\sigma} .
\end{aligned}
$$

É fácil verificar que $f$ é um isomorfismo de $\Lambda_{\overline{1}}$-módulos à esquerda.

Seja $\xi \in \Lambda_{\overline{1}}$. Observe que $\rho_{1}(\xi \sigma)=\xi \sigma \otimes \bar{\sigma}$ e $\rho_{2}(\xi \otimes \sigma)=(\xi \otimes \sigma) \otimes \bar{\sigma}$ definem estruturas de $k \overline{G(\Lambda)}$-comódulo à direita em $\Lambda_{\bar{\sigma}}$ e em $\Lambda_{\overline{1}} \otimes \bar{\sigma}$, respectivamente.

Além disso, temos que $\left(f \otimes \operatorname{Id}_{k} \overline{G(\Lambda)}\right) \circ \rho_{1}=\rho_{2} \circ f$, ou seja, $f$ é um morfismo de $k \overline{G(\Lambda)}$-comódulos à direita, na verdade um isomorfismo, como mostramos acima.

Segue um importante resultado extraído de [WY01, Corollary 2.3], que aborda a estrutura dos $H$-comódulo subálgebras de $K \# H$ contendo $K$.

Corolário 5.3.4. Seja $\Lambda$ um $H$-comódulo subálgebra de $K \# H$ contendo $K$. Então $\Lambda \cong \Lambda^{\prime} \#{ }_{\delta} k \overline{G(\Lambda)}$, onde $\Lambda^{\prime} \#_{\delta} k \overline{G(\Lambda)}$ é um produto cruzado.

Demonstração. Pelo Lema 5.3.3 (2), $\Lambda$ é uma álgebra fortemente $\overline{G(\Lambda)}$-graduada. Pelo Lema 5.3.2, temos que $\Lambda^{\prime}=\Lambda_{\overline{1}}$, e pelo Exemplo 1.3.12, obtemos $\Lambda^{\mathrm{co} H^{\prime}}=\Lambda^{\prime}$, onde $H^{\prime}=k \overline{G(\Lambda)}$. Daí, pelo Teorema 1.3.13, temos que $\Lambda^{\prime} \subseteq \Lambda$ é $k \overline{G(\Lambda)}$-Galois à direita. Além disso, pelo Lema 5.3.3 (5), temos que

$$
\Lambda=\bigoplus_{\bar{\sigma} \in \overline{G(\Lambda)}} \Lambda_{\bar{\sigma}} \cong \bigoplus_{\bar{\sigma} \in \overline{G(\Lambda)}}\left(\Lambda_{\overline{1}} \otimes \bar{\sigma}\right)=\Lambda_{\overline{1}} \otimes k \overline{G(\Lambda)}=\Lambda^{\prime} \otimes k \overline{G(\Lambda)},
$$

onde o isomorfismo é de $\Lambda^{\prime}$-módulos à esquerda e de $k \overline{G(\Lambda)}$-comódulos à direita. Assim, $\Lambda^{\prime}$ tem a propriedade da base normal à direita.

Finalmente, pelo Corolário 1.3.14, temos que $\Lambda \cong \Lambda^{\prime} \#_{\delta} k \overline{G(\Lambda)}$ como álgebras, onde $\Lambda^{\prime} \#_{\delta} k \overline{G(\Lambda)}$ é um produto cruzado.

Denotemos $\widehat{\varepsilon}=\operatorname{Id}_{K} \otimes \varepsilon$. Observe que $\widehat{\varepsilon} \in \operatorname{hom}(K \# H, K)$, e que

$\left(\widehat{\varepsilon} \otimes \operatorname{Id}_{H}\right) \circ \rho=\left(\operatorname{Id}_{K} \otimes \varepsilon \otimes \operatorname{Id}_{H}\right) \circ\left(\operatorname{Id}_{K} \otimes \Delta\right)=\operatorname{Id}_{K} \otimes\left[\left(\varepsilon \otimes \operatorname{Id}_{H}\right) \circ \Delta\right]=\operatorname{Id}_{K} \otimes \operatorname{Id}_{H}=\operatorname{Id}_{K \# H}$.

Antes do resultado mais importante desta seção, segue um lema a respeito da estrutura $K \# H^{K}$. 
Antes de mais nada, note que $k$ está imerso em $K$, e $K \# H^{K}$ pode ser visto como uma $K$-álgebra.

Lema 5.3.5. A $K$-álgebra $K \# H^{K}$ é uma álgebra de Hopf pontual de dimensão finita sobre o corpo $K$, onde $\Delta_{K \# H^{K}}=\rho=\operatorname{Id}_{K} \otimes \Delta, \varepsilon_{K \# H^{K}}=\widehat{\varepsilon} e S_{K \# H^{K}}=\operatorname{Id}_{K} \otimes S$.

Demonstração. Primeiramente, observe que, pelo Lema 2.2.3, $H^{K}$ age trivialmente em $K$. Assim, $\alpha h=h \alpha$, para todo $\alpha \in K$ e todo $h \in H^{K}$. Pela Proposição 5.2.3, sabemos que $H^{K}$ é uma subálgebra de Hopf de $H$. Como $H$ é uma álgebra de Hopf pontual de dimensão finita, $H^{K}$ também é uma álgebra de Hopf pontual de dimensão finita.

Seja $\left\{h_{i}\right\}$ uma base de $H^{K}$. Então $\left\{1 \# h_{i}\right\}$ é uma $K$-base de $K \# H^{K}$. Assim, é fácil mostrar que $K \# H^{K}$ é uma álgebra de Hopf de dimensão finita sobre o corpo $K$. Falta mostrar que $K \# H^{K}$ é uma álgebra de Hopf pontual.

Observe que podemos identificar $H^{K}$ com $1 \# H^{K}$ em $K \# H^{K}$. Seja $C$ uma subcoálgebra simples de $K \# H^{K}$ e seja $\xi=\sum \alpha_{j} \# h_{j} \in C$ tal que $\Delta\left(h_{j}\right)=\sum h_{i, j} \otimes h_{i}$, onde $h_{i, j} \in H^{K}$, para todo $i$ e todo $j$, e $\xi \neq 0$. Podemos escrever

$$
\Delta_{K \# H^{K}}(\xi)=\sum_{j} \alpha_{j} \# \Delta\left(h_{j}\right)=\sum_{j}\left[\alpha_{j} \#\left(\sum_{i} h_{i, j} \otimes_{K} h_{i}\right)\right]=\sum_{j, i}\left(\alpha_{j} \# h_{i, j}\right) \otimes_{K}\left(1 \# h_{i}\right) .
$$

Como $C$ é subcoálgebra e $\xi \neq 0$, temos que $\left(\alpha_{j} \# h_{i, j}\right) \otimes_{K}\left(1 \# h_{i}\right) \neq 0$, para algum par $i, j$. Como $\left\{1 \# h_{i}\right\}$ é $K$-linearmente independente, temos que $1 \# h_{i} \in C$, para algum $i$. Pelo fato de $C$ ser simples, a subcoálgebra gerada por $1 \# h_{i}$ é igual a $C$.

Identificando $1 \# h_{i}$ em $C$ com $h_{i}$ em $H^{K}$, é fácil ver que podemos identificar $C$ com a subcoálgebra $D$ gerada por $h_{i}$ em $H^{K}$. Se $D$ não for simples, concluiríamos que $C$ também não é simples, o que seria uma contradição. Assim, $D$ é simples. Do fato de $H^{K}$ ser pontual, $D$ é unidimensional. Logo, $C$ também é unidimensional.

Portanto, $K \# H^{K}$ é uma álgebra de Hopf pontual de dimensão finita sobre o corpo $K$

Definição. Seja $\eta \in \Lambda$. Dizemos que $\eta$ é um integral generalizado à esquerda de $\Lambda$ se $\xi \eta=\widehat{\varepsilon}(\xi) \eta$, para todo $\xi \in \Lambda$.

Finalmente, segue o resultado mais importante desta seção, extraído de [WY01, Proposition 2.5]. 
Proposição 5.3.6. $\quad$ 1. Seja $\Lambda$ um $H$-comódulo subálgebra de $K \# H$ contendo $K$. Então $\Lambda$ possui um integral generalizado à esquerda não nulo $\eta_{\Lambda}$.

2. Sejam $\Lambda_{1}$ e $\Lambda_{2}$ como $\Lambda$ acima. Se $\Lambda_{1} \subseteq \Lambda_{2}$, então $\eta_{\Lambda_{1}}=\eta_{\Lambda_{2}}$ implica em $\Lambda_{1}=\Lambda_{2}$.

Demonstração. (1) Pelo Lema 5.3.5, temos que $K \# H^{K}$ é uma álgebra de Hopf pontual de dimensão finita sobre o corpo $K$. Observe que $\Lambda^{\prime}=\Lambda \cap\left(K \# H^{K}\right)$ é um coideal subálgebra à direita de $K \# H^{K}$.

Pela Proposição 1.3.20, $K \# H^{K}$ possui a propriedade (Fr). Assim, $\Lambda^{\prime}$ é uma álgebra de Frobenius e, consequentemente, é uma álgebra quasi-Frobenius. Do Teorema 1.3.23, temos que existe um integral não nulo à esquerda $\eta^{\prime} \in \Lambda^{\prime}$. Pelo Corolário 1.3.24, $\eta^{\prime}$ é o único integral à esquerda de $\Lambda^{\prime}$, a menos de multiplicação por escalar do corpo base $K$.

Denotemos $t_{G(\Lambda)}=\sum_{\sigma \in G(\Lambda)} \sigma$. Vamos mostrar que $\eta_{\Lambda}=t_{G(\Lambda)} \eta^{\prime}$. Seja $\sigma \in G(\Lambda)$ e sejam $\xi, \xi^{\prime} \in \Lambda$. Vamos definir a seguinte operação de $k G(\Lambda) \times \Lambda$ em $\Lambda$ :

$$
\sigma * \xi=\sigma \xi \sigma^{-1}
$$

É fácil ver que $\Lambda$ é um $k G(\Lambda)$-módulo álgebra à esquerda via operação definida acima. Pela Proposição 5.2.3, sabemos que $H^{K}$ é uma subálgebra de Hopf normal de $H\left(\operatorname{ad}_{1}\left(H, H^{K}\right) \subseteq H^{K}\right.$ e $\left.\operatorname{ad}_{\mathrm{r}}\left(H, H^{K}\right) \subseteq H^{K}\right)$. Assim, $\Lambda^{\prime}$ é $k G(\Lambda)$-estável, ou seja, $k G(\Lambda) * \Lambda^{\prime} \subseteq \Lambda^{\prime}$. De fato, seja $\sigma \in G(\Lambda)$ e seja $\xi^{\prime}=\sum \alpha_{i} \# h_{i} \in \Lambda^{\prime}$, onde $\alpha_{i} \# h_{i} \in K \# H^{K}$, para todo $i$. Lembre que $\Lambda$ é uma subálgebra de $K \# H$ contendo $K$, e que $K$ é estável sob a ação de $H$. Então:

$$
\sigma * \xi^{\prime}=\sigma \sum\left(\alpha_{i} \# h_{i}\right) \sigma^{-1}=\sum \sigma \cdot \alpha_{i} \# \sigma h_{i} \sigma^{-1}=\sum \sigma \cdot \alpha_{i} \# \operatorname{ad}_{l}\left(\sigma, h_{i}\right) \in \Lambda \cap\left(K \# H^{K}\right)=\Lambda^{\prime} .
$$

Inicialmente, vamos mostrar que $\eta_{\Lambda} \neq 0$. Pelo Lema $5.3 .3(1)$, temos que $\overline{G(\Lambda)}=G(\Lambda) / G\left(\Lambda^{\prime}\right)$. Do fato de $G\left(\Lambda^{\prime}\right)$ e $\overline{G(\Lambda)}$ serem grupos finitos, podemos escrever $G\left(\Lambda^{\prime}\right)=\left\{\tau_{1}=1, \cdots, \tau_{m}\right\}$, e $\overline{G(\Lambda)}=\left\{\overline{\sigma_{1}}=\overline{1}, \cdots, \overline{\sigma_{n}}\right\}$, onde $\sigma_{i} \in G(\Lambda)$, e $\overline{\sigma_{i}} \neq \overline{\sigma_{j}}$, onde $i \neq j$, para todos $1 \leq i, j \leq n$. Assim, como $G\left(\Lambda^{\prime}\right)$ é subgrupo normal de $G(\Lambda)$, é fácil ver que $G(\Lambda)=\left\{\sigma_{i} \tau_{j}: 1 \leq i \leq n\right.$ e $\left.1 \leq j \leq m\right\}$.

Temos então que

$$
\eta_{\Lambda}=t_{G(\Lambda)} \eta^{\prime}=\sum_{i, j} \sigma_{i} \tau_{j} \eta^{\prime}=m \sum_{i} \sigma_{i} \eta^{\prime}=m \sum_{i}\left(\sigma_{i} \eta^{\prime} \sigma_{i}^{-1}\right) \sigma_{i}
$$


Como $\Lambda^{\prime}$ é $k G(\Lambda)$-estável, temos que $\sigma_{i} \eta^{\prime} \sigma_{i}^{-1} \in \Lambda^{\prime}$, para todo $1 \leq i \leq n$.

Pelo Corolário 5.3.4, temos que $\Lambda \cong \Lambda^{\prime} \# \#_{\delta} \overline{G(\Lambda)}$. Assim, podemos identificar $\eta_{\Lambda} \in \Lambda$ com o elemento $\sum_{i} m\left(\sigma_{i} \eta^{\prime} \sigma_{i}^{-1}\right) \#_{\delta} \overline{\sigma_{i}} \in \Lambda^{\prime} \#_{\delta} k \overline{G(\Lambda)}$. Vamos supor que $\eta_{\Lambda}=0$. Pelo fato da característica do corpo base $k$ ser igual a zero, temos que $\sigma_{i} \eta^{\prime} \sigma_{i}^{-1}=0$, para todo $1 \leq i \leq n$. Logo, $\eta^{\prime}=0$, o que seria um absurdo. Portanto, $\eta_{\Lambda} \neq 0$.

Assim, para todo $\xi^{\prime}=\sum_{i} \alpha_{i} \# h_{i} \in \Lambda^{\prime}$, temos que

$$
\begin{aligned}
\xi^{\prime} \eta_{\Lambda} & =\sum_{\sigma \in G(\Lambda)} \xi^{\prime} \sigma \eta^{\prime}=\sum_{\sigma \in G(\Lambda)} \sigma\left(\sigma^{-1} * \xi^{\prime}\right) \eta^{\prime} \\
& =\sum_{\sigma \in G(\Lambda)} \sigma \widehat{\varepsilon}\left(\sigma^{-1} * \xi^{\prime}\right) \eta^{\prime} \text {, pois } \eta^{\prime} \text { é integral à esquerda em } \Lambda^{\prime} \\
& =\sum_{\sigma \in G(\Lambda)} \sigma \widehat{\varepsilon}\left(\sum_{i} \sigma^{-1} \cdot \alpha_{i} \# \sigma^{-1} h_{i} \sigma\right) \eta^{\prime} \\
& =\sum_{\sigma \in G(\Lambda)} \sigma\left(\sum_{i} \sigma^{-1} \cdot \alpha_{i} \# \varepsilon\left(h_{i}\right)\right) \eta^{\prime} \\
& =\sum_{\sigma \in G(\Lambda)}\left[\sum_{i}\left(\sigma \cdot\left(\sigma^{-1} \cdot \alpha_{i}\right)\right) \# \sigma \varepsilon\left(h_{i}\right)\right] \eta^{\prime} \\
& =\sum_{\sigma \in G(\Lambda)}\left[\sum_{i} \alpha_{i} \# \varepsilon\left(h_{i}\right)\right] \sigma \eta^{\prime} \\
& =\sum_{\sigma \in G(\Lambda)} \widehat{\varepsilon}\left(\xi^{\prime}\right) \sigma \eta^{\prime} \\
& =\widehat{\varepsilon}\left(\xi^{\prime}\right) \eta_{\Lambda} .
\end{aligned}
$$

Além disso, pelo Lema 5.3.2, temos que $\Lambda^{\prime}=\Lambda_{\overline{1}}$, e pelo Lema 5.3.3, temos que

$$
\Lambda=\bigoplus_{\bar{\sigma} \in \overline{G(\Lambda)}} \Lambda_{\bar{\sigma}}=\bigoplus_{\bar{\sigma} \in \overline{G(\Lambda)}}\left(\Lambda_{\overline{1}} \sigma\right)=\bigoplus_{\bar{\sigma} \in \overline{G(\Lambda)}}\left(\Lambda^{\prime} \sigma\right)
$$


Seja $\sigma \in G(\Lambda)$ e seja $\xi \in \Lambda_{\bar{\sigma}}$. Podemos escrever, $\xi=\xi^{\prime} \sigma$, para algum $\xi^{\prime} \in \Lambda^{\prime}$. Então:

$$
\xi \eta_{\Lambda}=\xi^{\prime} \sigma t_{G(\Lambda)} \eta^{\prime}=\xi^{\prime} t_{G(\Lambda)} \eta^{\prime}=\xi^{\prime} \eta_{\Lambda}=\widehat{\varepsilon}\left(\xi^{\prime}\right) \eta_{\Lambda}=\widehat{\varepsilon}\left(\xi^{\prime} \sigma\right) \eta_{\Lambda}=\widehat{\varepsilon}(\xi) \eta_{\Lambda}
$$

Logo, $\eta_{\Lambda}$ é um integral generalizado à esquerda não nulo de $\Lambda$.

(2) Vamos assumir que $\eta=\eta_{\Lambda_{1}}=\eta_{\Lambda_{2}}$.

Pelo Corolário 5.3.4, temos que $\Lambda_{1} \cong \Lambda_{1}^{\prime} \# \delta_{\delta_{1}} k \overline{G\left(\Lambda_{1}\right)}$ e que $\Lambda_{2} \cong \Lambda_{2}^{\prime} \# \delta_{2} k \overline{G\left(\Lambda_{2}\right)}$. Observe que $\Lambda_{1}^{\prime} \subseteq \Lambda_{2}^{\prime}$ e que $\overline{G\left(\Lambda_{1}\right)} \subseteq \overline{G\left(\Lambda_{2}\right)}$. Se $\Lambda_{1} \neq \Lambda_{2}$, então pelo menos uma das inclusões acima é estrita.

Além disso, note que $\Lambda_{1}^{\prime}$ é um coideal subálgebra à direita de $K \# H^{K}$. Novamente pela Proposição 1.3.20, $K \# H^{K}$ possui a propriedade (Fr). Assim, $\Lambda_{1}^{\prime}$ é uma álgebra de Frobenius. Como $\Lambda_{2}^{\prime}$ tem estrutura de $K \# H^{K}$-comódulo à direita e de $\Lambda_{1}^{\prime}$-módulo à esquerda e à direita, pelo Teorema 1.3.21, temos que $\Lambda_{2}^{\prime}$ é um $\Lambda_{1}^{\prime}$-módulo à esquerda livre e um $\Lambda_{1}^{\prime}$-módulo à direita livre.

Vamos supor que $\Lambda_{2}^{\prime}$, como um $\Lambda_{1}^{\prime}$-módulo à direita livre, tenha dimensão maior do que um. Assim, existem $\xi_{1}, \xi_{2} \in \Lambda_{2}^{\prime}$ tais que $\left\{\xi_{1}, \xi_{2}\right\}$ é $\Lambda_{1}^{\prime}$-linearmente independente. Podemos escrever $\xi_{1}=$ $\sum \alpha_{i} \# x_{i}$ e $\xi_{2}=\sum \beta_{j} \# y_{j}$, onde $\alpha_{i} \# x_{i}, \beta_{j} \# y_{j} \in K \# H^{K}$, para todo $i$ e todo $j$.

Denotemos $\alpha=\sum \varepsilon\left(x_{i}\right) \alpha_{i}$ e $\beta=\sum \varepsilon\left(y_{j}\right) \beta_{j}$. Observe que $\alpha, \beta \neq 0$. De fato, se $\alpha=0$, então $\xi_{1} \eta+\xi_{2} 0=\widehat{\varepsilon}\left(\xi_{1}\right) \eta=\alpha \eta=0$, o que é uma contradição. De forma análoga, mostra-se que $\beta \neq 0$. Então:

$$
\begin{aligned}
\xi_{1}\left(\alpha^{-1} \beta\right) \eta+\xi_{2}(-\eta) & =\sum\left[\alpha_{i}\left(x_{i_{(1)}} \cdot\left(\alpha^{-1} \beta\right)\right) \# x_{i_{(2)}}\right] \eta-\widehat{\varepsilon}\left(\xi_{2}\right) \eta \\
& =\sum\left[\alpha_{i}\left(\alpha^{-1} \beta\right) \# x_{i}\right] \eta-\beta \eta, \text { pois } H^{K} \text { age trivialmente em } K \\
& =\left(\alpha^{-1} \beta\right)(\xi \eta)-\beta \eta \\
& =\left(\alpha^{-1} \beta\right)(\alpha \eta)-\beta \eta=\beta \eta-\beta \eta=0 .
\end{aligned}
$$

Assim, $\left\{\xi_{1}, \xi_{2}\right\}$ é $\Lambda_{1}^{\prime}$-linearmente dependente, um absurdo. Logo, $\Lambda_{2}^{\prime}$ tem dimensão um como um $\Lambda_{1}^{\prime}$-módulo à direita livre. Como $\Lambda_{1}^{\prime} \subseteq \Lambda_{2}^{\prime}$, temos que $\Lambda_{1}^{\prime}=\Lambda_{2}^{\prime}$.

De forma análoga, note que $k G\left(\Lambda_{1}\right)$ é um coideal subálgebra à direita de $k G(H)$. Pela Proposição 1.3.20, $k G(H)$ possui a propriedade (Fr). Assim, $k G\left(\Lambda_{1}\right)$ é uma álgebra de Frobenius. Como $k G\left(\Lambda_{2}\right)$ tem estrutura de $k G(H)$-comódulo à direita e de $k G\left(\Lambda_{1}\right)$-módulo à esquerda e à direita, pelo Teorema 
1.3.21, temos que $k G\left(\Lambda_{2}\right)$ é um $k G\left(\Lambda_{1}\right)$-módulo à esquerda livre e um $k G\left(\Lambda_{1}\right)$-módulo à direita livre.

Vamos supor que $k G\left(\Lambda_{2}\right)$, como um $k G\left(\Lambda_{1}\right)$-módulo à direita livre, tenha dimensão maior do que um. Assim, existem $\xi_{1}, \xi_{2} \in k G\left(\Lambda_{2}\right)$ tais que $\left\{\xi_{1}, \xi_{2}\right\}$ é $k G\left(\Lambda_{1}\right)$-linearmente independente. Podemos escrever $\xi_{1}=\sum \lambda_{i} \sigma_{i}$ e $\xi_{2}=\sum \lambda_{j}^{\prime} \tau_{j}$, onde $\lambda_{i}, \lambda_{j}^{\prime} \in k$ e $\sigma_{i}, \tau_{j} \in G\left(\Lambda_{2}\right)$, para todo $i$ e todo $j$.

Denotemos $\lambda=\sum \lambda_{i}$ e $\lambda^{\prime}=\sum \lambda_{j}^{\prime}$. Observe que $\lambda, \lambda^{\prime} \neq 0$. De fato, se $\lambda=0$, então $\xi_{1} \eta+\xi_{2} 0=$ $\widehat{\varepsilon}\left(\xi_{1}\right) \eta=\lambda \eta=0$, o que é uma contradição. De forma análoga, mostra-se que $\lambda^{\prime} \neq 0$. Então:

$$
\xi_{1}\left(\lambda^{-1} \lambda^{\prime}\right) \eta+\xi_{2}(-\eta)=\left(\lambda^{-1} \lambda^{\prime}\right) \xi_{1} \eta-\widehat{\varepsilon}\left(\xi_{2}\right) \eta=\left(\lambda^{-1} \lambda^{\prime}\right) \widehat{\varepsilon}\left(\xi_{1}\right) \eta-\lambda^{\prime} \eta=\left(\lambda^{-1} \lambda^{\prime}\right) \lambda \eta-\lambda^{\prime} \eta=0 .
$$

Assim, $\left\{\xi_{1}, \xi_{2}\right\}$ é $k G\left(\Lambda_{1}\right)$-linearmente dependente, um absurdo. Logo, $k G\left(\Lambda_{2}\right)$ tem dimensão um como um $k G\left(\Lambda_{1}\right)$-módulo à direita livre. Como $k G\left(\Lambda_{1}\right) \subseteq k G\left(\Lambda_{2}\right)$, temos que $k G\left(\Lambda_{1}\right)=k G\left(\Lambda_{2}\right)$. Assim, $\overline{G\left(\Lambda_{1}\right)}=\overline{G\left(\Lambda_{2}\right)}$.

Finalmente, concluímos que $\Lambda_{1}=\Lambda_{2}$.

\subsection{O teorema}

Antes de mostrar o resultado principal deste capítulo, segue um lema extraído de [Yan97, Lemma $5.5]$.

Lema 5.4.1. Seja $R$ uma álgebra prima e seja $H$ uma álgebra de Hopf pontual de dimensão finita que age em $R$. Assuma que a ação de $H$ em $R$ é X-externa. Seja $\Lambda$ um subconjunto não nulo de $K \# H$. Então $R^{\Lambda}$ é uma subálgebra de $R$ racionalmente completa contendo $R^{H}$.

Demonstração. Pelo Lema 3.1.1, temos que $R^{H}=\{r \in R: h \cdot r=\varepsilon(h) r$, para todo $h \in H\}$. Seja $\xi=\sum \alpha_{i} \# h_{i} \in \Lambda$ e seja $x \in R^{H}$. Então:

$$
\xi x=\left(\sum \alpha_{i} \# h_{i}\right) x=\sum \alpha_{i}\left(h_{i_{(1)}} \cdot x\right) \# h_{i_{(2)}}=\sum \alpha_{i} x \# h_{i}=x \sum \alpha_{i} \# h_{i}=x \xi .
$$

Da igualdade acima, temos que $R^{H} \subseteq R^{\Lambda}$.

Seja $I$ um ideal não nulo de $R^{\Lambda}$ e seja $r \in R$. Vamos assumir que $r I \subseteq R^{\Lambda}$. Então, temos que 
$\xi(r I)=(r I) \xi=r \xi I$, ou seja, $(\xi r-r \xi) I=0$. Como $R^{\Lambda} I \subseteq I$, temos que $(\xi r-r \xi) R^{\Lambda} I=0$. Pela Proposição 3.4.4 (2), temos que $\xi r-r \xi=0$, ou seja, $r \in R^{\Lambda}$.

Logo, $R^{\Lambda}$ é uma subálgebra de $R$ racionalmente completa contendo $R^{H}$.

Finalmente, estamos em condições de mostrar a sobrejetividade da correspondência e assim concluir a demonstração do teorema. Resultado extraído de [WY01, Theorem 2.6].

Teorema 5.4.2. Seja $R$ uma álgebra prima e seja $H$ uma álgebra de Hopf pontual de dimensão finita que age em $R$. Assuma que a característica do corpo base $k$ é zero e que a ação de $H$ em $R$ é $X$-externa. Então, para cada $H$-comódulo subálgebra $\Lambda$ de $K \# H$ que contenha $K$, temos que $\Lambda=\Phi\left(R^{\Lambda}\right)$.

Demonstração. Vamos denotar $U=R^{\Lambda}$. Pelo Lema 5.4.1, $U$ é uma subálgebra de $R$ racionalmente completa contendo $R^{H}$. É claro que $\Lambda \subseteq \Phi(U)=(K \# H)^{U}$. Note que, pela Proposição 3.4.4 (7), $(K \# H)^{U}$ é um $H$-comódulo subálgebra de $K \# H$. Assim, pela Proposição 5.3.6 (1), $\Lambda$ possui um integral generalizado à esquerda não nulo $\eta_{\Lambda}$. Pela Proposição 5.3.6 (2), se mostrarmos que $\eta_{\Lambda}$ é também um integral generalizado à esquerda não nulo de $\Phi(U)$, então $\Lambda=\Phi(U)$.

Seja $I$ um ideal não nulo de $R$. Pela Proposição 3.4.4 (1), temos que $\eta_{\Lambda} \cdot I \neq 0$. Vamos mostrar que $\eta_{\Lambda} \cdot I \subseteq U$. De fato, seja $x \in I$ e seja $\xi \in \Lambda$. Podemos escrever $\rho(\xi)=\sum \xi_{i} \otimes h_{i}$, onde $\xi_{i} \in \Lambda$ e $h_{i} \in H$, para todo $i$. Então:

$\xi\left(\eta_{\Lambda} \cdot x\right)=\sum \xi_{i} \cdot\left(\eta_{\Lambda} \cdot x\right) \# h_{i}=\sum\left(\xi_{i} \eta_{\Lambda}\right) \cdot x \# h_{i}=\sum\left(\widehat{\varepsilon}\left(\xi_{i}\right) \eta_{\Lambda}\right) \cdot x \# h_{i}=\sum\left(\eta_{\Lambda} \cdot x\right) \widehat{\varepsilon}\left(\xi_{i}\right) \# h_{i}=\left(\eta_{\Lambda} \cdot x\right) \xi$

Seja $\xi^{\prime} \in \Phi(U)$. Podemos escrever $\rho\left(\xi^{\prime}\right)=\sum \xi_{j}^{\prime} \otimes h_{j}^{\prime}$, onde $\xi_{j}^{\prime} \in \Phi(U)$ e $h_{j}^{\prime} \in H$, para todo $j$, e $\left\{h_{j}^{\prime}\right\}$ é linearmente independente. Então, $\xi^{\prime}\left(\eta_{\Lambda} \cdot x\right)=\left(\eta_{\Lambda} \cdot x\right) \xi^{\prime}$. Pela expressão acima, temos que

$$
\sum\left(\xi_{j}^{\prime} \eta_{\Lambda}\right) \cdot x \# h_{j}^{\prime}=\sum\left(\widehat{\varepsilon}\left(\xi_{j}^{\prime}\right) \eta_{\Lambda}\right) \cdot x \# h_{j}^{\prime}
$$

Assim, $\left(\xi_{j}^{\prime} \eta_{\Lambda}\right) \cdot x=\left(\widehat{\varepsilon}\left(\xi_{j}^{\prime}\right) \eta_{\Lambda}\right) \cdot x$, ou seja, $\left(\xi_{j}^{\prime} \eta_{\Lambda}-\widehat{\varepsilon}\left(\xi_{j}^{\prime}\right) \eta_{\Lambda}\right) \cdot I=0$. Pela Proposição 3.4 .4 (1), segue que $\xi_{j}^{\prime} \eta_{\Lambda}=\widehat{\varepsilon}\left(\xi_{j}^{\prime}\right) \eta_{\Lambda}$, para todo $j$.

Como $\xi^{\prime}=\sum \varepsilon\left(h_{j}^{\prime}\right) \xi_{j}^{\prime}$, temos que

$$
\xi^{\prime} \eta_{\Lambda}=\sum \varepsilon\left(h_{j}^{\prime}\right) \xi_{j}^{\prime} \eta_{\Lambda}=\sum \varepsilon\left(h_{j}^{\prime}\right) \widehat{\varepsilon}\left(\xi_{j}^{\prime}\right) \eta_{\Lambda}=\sum \widehat{\varepsilon}\left(\varepsilon\left(h_{j}^{\prime}\right) \xi_{j}^{\prime}\right) \eta_{\Lambda}=\widehat{\varepsilon}\left(\xi^{\prime}\right) \eta_{\Lambda} .
$$


Assim, mostramos que $\eta_{\Lambda}$ é também um integral generalizado à esquerda não nulo de $\Phi(U)$. Logo, $\Lambda=\Phi(U)$.

\subsection{Considerações finais}

Finalmente, após um longo percurso, podemos juntar o Corolário 4.2.6 e o Teorema 5.4.2 e obter a correspondência do tipo Galois para ações de álgebras de Hopf pontuais de dimensão finita em álgebras primas. Segue novamente o enunciado deste teorema da correspondência:

Teorema 5.5.1. Seja $k$ um corpo de característica zero e seja $H$ uma álgebra de Hopf pontual de dimensão finita sobre $k$ agindo em uma $k$-álgebra prima $R$ de modo que a ação seja X-externa. Denotemos por $Q$ a álgebra de quocientes de Martindale simétrica de $R$ e por $K$ o centróide estendido de $R$. Seja $\mathcal{S}$ o conjunto de todos os $H$-comódulo subálgebras $\Lambda$ de $K \# H$ contendo $K$, e seja $\mathcal{F}$ o conjunto de todas as subálgebras racionalmente completas $U$ de $R$ contendo $R^{H}$. Então a aplicação

$$
\begin{array}{rlc}
\Phi: \mathcal{F} & \longrightarrow & \mathcal{S} \\
U & \longmapsto(K \# H)^{U}
\end{array}
$$

é bijetora com inversa dada por

$$
\begin{aligned}
\Psi: \mathcal{S} & \longrightarrow \mathcal{F} \\
\Lambda & \longmapsto R^{\Lambda}
\end{aligned}
$$

Observação. $\Phi\left(R^{H}\right)=(K \# H)^{R^{H}}=K \# H$ e $\Phi(R)=(K \# H)^{R}=K$.

Segue um exemplo da correspondência extraído de [Yan05, Example 2.8 and Example 2.14]. Convém ressaltar que os detalhes das demonstrações deste exemplo serão omitidos. A idéia seria apenas ilustrar a correspondência demonstrada nesta dissertação.

Exemplo 5.5.2. Seja $R=k\langle v, \nu\rangle$ a álgebra gerada pelas variáveis indeterminadas não comutativas $v, \nu$ sobre $k$. Então, $R$ é uma álgebra prima e a álgebra de quocientes de Martindale simétrica de $R$ é o próprio $R$. O centróide extendido de $R$ é $k$, ou seja, $K=k$.

Considere $H=H_{4}$ a álgebra de Hopf de Sweedler de dimensão quatro, isto, é

$$
H_{4}=k\left\langle X, Y: X^{2}=1, Y^{2}=0, Y X=-X Y\right\rangle \text {. }
$$


A estrutura de coálgebra de $H_{4}$ é dada por

$$
\Delta(X)=X \otimes X, \quad \Delta(Y)=Y \otimes 1+X \otimes Y \quad \text { e } \quad \varepsilon(X)=1, \quad \varepsilon(Y)=0 .
$$

Vamos definir a ação de $H$ em $R$ da seguinte forma:

$$
X \cdot v=\nu, \quad X \cdot \nu=v, \quad Y \cdot v=1 \text { e } Y \cdot \nu=-1
$$

Temos que ação de $H$ em $R$ é X-externa. Utilizando a mesma notação do Teorema 5.5.1, temos a correspondência ilustrada na tabela abaixo:

\begin{tabular}{lll}
\hline$\Lambda$ & $\Psi(\Lambda)=R^{\Lambda}$ & $\eta_{\Lambda}$ \\
\hline$K$ & $R$ & $1_{K}$ \\
$K\langle X\rangle$ & $\{r \in R: X \cdot r=r\}$ & $1+X$ \\
$K\langle\alpha X+Y\rangle$ & $\{r \in R: Y \cdot r=\alpha(r-X \cdot r)\}$ & $\alpha X+Y+\alpha$ \\
$K \# H$ & $\{r \in R: X \cdot r=r \mathrm{e} Y \cdot r=0\}$ & $X Y+Y$ \\
\hline
\end{tabular}

É importante dizer que, em [MY03, Theorem 3.5], Masuoka e Yanai eliminam a restrição da característica de $k$ ser zero. Como uma aplicação deste teorema mais geral de Masuoka e Yanai, podemos citar o artigo [FMP04], que mostra uma correspondência do tipo Galois entre coideais subálgebras à direita de uma álgebra de Hopf pontual de dimensão finita, que age de forma homogênea e fiel em uma álgebra associativa livre, e subálgebras livres contendo os invariantes desta ação. 


\section{Referências Bibliográficas}

[Coh86] M. Cohen, Smash products, inner actions and quotient rings, Pacific J. Math. 125 (1986), no. $1,45-66$. xii, 29

[DNR00] S. Dascalescu, C. Nastasescu, and S. Raianu, "Hopf Algebras: An Introduction", Marcel Dekker, New York, 2000. 5, 20, 24, 26

[FM06] V. O. Ferreira and L. S. I. Murakami, "Introdução às álgebras de Hopf", apostila para curso ministrado no IME-USP, São Paulo, 2006. 5

[FMP04] V. O. Ferreira, L. S. I. Murakami, and A. Paques, A Hopf-Galois correspondence for free algebras, J. Algebra 276 (2004), 407-416. 105

[Kha91] V. K. Kharchenko, "Automorphisms and Derivations of Associative Rings", Kluwer Academic, Dordrecht, 1991. xi

[Kop93] M. Koppinen, Coideal subalgebras in Hopf algebras: Freeness, integrals, smash products, Comm. Algebra 21 (1993), no. 2, 427-444. 26

[Mar85] T. Marlowe, The diagonal of a pointed coalgebra and incidence-like structure, J. Pure and Appl. Algebra 35 (1985), 157-169. 38, 40

[Mas92] A. Masuoka, Freeness of Hopf algebras over coideal subalgebras, Comm. Algebra 20 (1992), no. $5,1353-1373.24$

[Mas94] _ C Coideal subalgebras in finite Hopf algebras, J. Algebra 163 (1994), 819-831. 24

[Mil95a] A. Milinski, Actions of pointed Hopf algebras on prime algebras, Comm. Algebra 23 (1995), no. $1,313-333$. xi, 50, 52, 54, 56, 58, 62, 63, 66, 70

[Mil95b]__ Operationen punktierter Hopfalgebren auf primen algebren, Ph.D. thesis, LudwigMaximilians-Universität München, 1995. xii, 68

[Mon93a] S. Montgomery, Biinvertible actions of Hopf algebras, Israel J. Math. 83 (1993), 45-71. 29 
[Mon93b] _ _Hopf Algebras and Their Actions on Rings", American Mathematical Society, Providence, 1993. 5, 13, 15, 17, 22, 23, 25, 38

[MY03] A. Masuoka and T. Yanai, Hopf module duality applied to X-outer galois theory, J. Algebra 265 (2003), 229-246. 105

[Pas87] D. S. Passman, Computing the symmetric ring of quotients, J. Algebra 105 (1987), 207235. 27,28

[Pas89] _ _ "Infinite Crossed Product", Academic Press, Boston, 1989. 28

[Swe69] M. E. Sweedler, "Hopf Algebras", W. A. Benjamin, New York, 1969. 5, 8, 9, 12, 22

[Wes99] S. Westreich, A Galois-type correspondence theory for actions of finite-dimensional pointed Hopf algebras on prime algebras, J. Algebra 219 (1999), 606-624. 70

[Wes00] , Inner and Outer Actions of Pointed Hopf Algebras, Contemporary Mathematics 267 (2000), 325-337. 36, 84, 87, 88

[WY01] S. Westreich and T. Yanai, More about a Galois-Type Correspondence Theory, J. Algebra 246 (2001), 629-640. 73, 74, 76, 78, 80, 81, 83, 92, 93, 97, 98, 103

[Yan97] T. Yanai, Correspondence theory of Kharchenko and X-outer actions of pointed Hopf algebras, Comm. Algebra 25 (1997), no. 6, 1713-1740. xii, 47, 56, 60, 66, 67, 102

[Yan05]__ Galois correspondence theorem for Hopf algebra actions, Contemporary Mathematics 376 (2005), 393-411. 81, 104 


\section{Índice Remissivo}

álgebra, 2, 6

de Frobenius, 24

de grupo, 5

de Hopf, 5, 12

de quocientes de Martindale, 27

à direita, 28

à esquerda, 28

simétrica, 28

prima, 3

quasi-Frobenius, 24

ação

adjunta à direita, 14

adjunta à esquerda, 14

contínua, 29

de álgebras de Hopf, 14

de grupo por automorfismos, 56

em álgebra prima, 29

X-externa, 56

antimorfismo

de álgebras, 12

de coálgebras, 12

aplicação twist, 4

biálgebra, 9

centróide estendido, 29

co-radical, 22

coálgebra, 6

co-oposta, 7

pontual, 22, 38 simples, 22

coassociatividade, 6

cociclo, 17

coideal, 7

à direita, 7

à esquerda, 7

subálgebra à direita, 13

subálgebra à esquerda, 13

comódulo

álgebra à direita, 21

à direita, 18

comultiplicação, 6

co-oposta, 7

counidade, 6

elemento

group-like, 8

group-like distinguido, 26

primitivo, 8,10

extensão

à direita, 21

Galois à direita, 22

filtração co-radical, 23

grau de elemento de coálgebra, 23

ideal

de Hopf, 12

integral, 25

de coideal subálgebra à direita, 26 
generalizado à esquerda, 98

módulo

à direita artiniano, 4

à direita injetivo, 4

à esquerda, 18

à esquerda artiniano, 4

à esquerda injetivo, 3

mede, veja ação de álgebras de Hopf

morfismo

álgebras, 2

de álgebras de Hopf, 12

de biálgebras, 11

de coálgebras, 7

de coḿódulos, 20

produto

cruzado, 17

de convolução, 11

smash, 16

propriedade da base normal à direita, 22

subálgebra

de coinvariantes, 21

de Hopf, 13

de Hopf normal, 14

de invariantes, 14

racionalmente completa, 3

subcoálgebra, 7

Teorema

de Taft-Wilson, 38

Fundamental das Coálgebras, 20

Fundamental dos Comódulos, 20 